MODELING, IDENTIFICATION AND CONTROL, 1999, VOL. 20, NO. 2, 83-134

doi:10.4173/mic.1999.23

\title{
Tutorial on Nonlinear Backstepping: Applications to Ship Control
}

\author{
THOR I. FOSSEN† and JANN PETER STRAND*
}

Keywords: Nonlinear control, backstepping, feedback linearization

\begin{abstract}
The theoretical foundation of nonlinear backstepping designs is presented in a tutorial setting. This includes a brief review of integral backstepping, extensions to SISO and MIMO systems in strict feedback form and physical motivated case studies. Parallels and differences to feedback linearization where it is shown how so-called "good nonlinearities" can be exploited in the design are also made.

Nonlinear, optimal and robust backstepping are discussed in a separate section where parallels to linear quadratic optimal control and $\boldsymbol{H}_{\infty}$-control are drawn. In addition, inverse optimality is discussed as a nonlinear design tool.

Physics is put into control by using mechanical systems like mass-damper-springs and ship models in the case studies. Lyapunov theory is used to prove convergence and stability for all control laws where energy dissipation is obtained by exploiting physical model properties.
\end{abstract}

\section{The idea of integral backstepping}

\subsection{A brief history of backstepping}

Backstepping is a recursive design methodology for construction of both feedback control laws and associated Lyapunov functions in a systematic manner. Nonlinear backstepping designs are strongly related to feedback linearization. However, while feedback linearization methods cancel all nonlinearities in the system it will be shown that when applying the backstepping design methodology the designer obtain flexibility to exploit "good" nonlinearities while "bad" or destabilizing nonlinearities are dominated e.g. by adding nonlinear damping. Hence, additional robustness is obtained. This is important in industrial control systems since cancellation of all nonlinearities require precise models which are difficult to obtain in practise.

The idea of integrator backstepping seems to have appeared simultaneously, often implicit, in the works of Koditschek (1987), Sonntag and Sussmann (1988), Tsinias (1989), and Byrnes and Isidori (1989). Stabilization through an integrator (Kokotovic and Sussmann, 1989) can be viewed as a special case of stabilization through an SPR transfer function which is a frequently used technique in the early adaptive designs, see Parks (1966), Landau (1979) and Narendra and Annaswamy (1989), for instance. Extensions to nonlinear cascades by using passivity arguments have been done by Ortega (1991) and Byrnes, Isidori and Willems (1991). Integrator backstepping appeared as a recursive design technique in Saberi, Kokotovic and Sussmann (1990) and it was further developed by Kanellakopoulos, Kokotovic and Morse (1992). The relationship between backstepping and passivity has been established by Lozano, Brogliato and Landau (1992). For the interested reader, a tutorial overview of backstepping is given by Kokotovic (1991).

†Department of Engineering Cybernetics, Norwegian University of Science and Technology, N-7491 Trondheim, Norway.

*ABB Industri AS, Hasleveien 50, Postboks 6540 Rodeløkka, N-0501 Oslo, Norway.

This paper is a slight revision of a paper presented in a tutorial session at IFAC Conference on Control Applications in Marine Systems (CAMS'98), Fukuoka, Japan, 27-30 October 1998. 


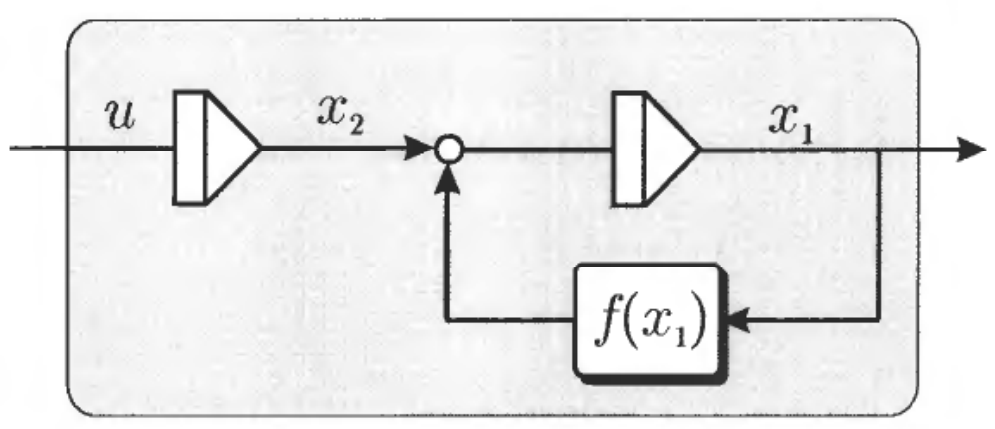

Figure 1. 2nd-order nonlinear system with one single nonlineraity $f\left(x_{1}\right)$ and a pure integrator at the input.

The first book describing the backstepping design methodology was published by Krstic, Kanellakopoulos and Kokotovic (1995). In this book emphasis is placed on adaptive and nonlinear control of SISO systems with extensions to MIMO systems in component form. Parameter adaptation, tuning functions and modular designs for both full state feedback and output feedback are also discussed.

Later a second book was published by Sepulchre, Jankovic and Kokotovic (1997). This textbook is an extension to forwarding, passivity and cascaded designs. Stability margins and optimality are also given a more detailed discussion.

More recently Krstic and Deng (1998) published a book with extension to stochastic systems focusing on stochastic stability and regulation, stochastic adaptive backstepping designs and disturbance attenuation.

The main difference of the material presented in the forthcoming sections and existing textbooks are that the focus is made towards practical designs, implementation considerations and examples based on mechanical systems and ship applications. Full-scale experiments with tankers and supply vessels are used to demonstrate the performance of the control systems. This is done by exploiting the nonlinear system properties of mechanical systems like dissipativness (good damping), symmetry of the inertia matrix and the skew-symmetric property of the Coriolis and centripetal matrix. In addition, emphasis is placed on control design with integral action. Two techniques for integral action in nonlincar systcms using backstepping designs are discussed (Fossen, Loria and Teel, 1999) and a detailed analysis of the different techniques where so-called good damping in mechanical systems is exploited. All results are presented in a vectorial setting in order to exploit the structural properties of nonlinear MIMO systems. This technique is referred to as vectorial backstepping, see Fossen and Berge (1997) and Fossen and Grøvlen (1998). Backstepping designs applied to ship control are described more closely in Strand (1999).

\subsection{Integrator backstepping}

The main idea of integrator backstepping can be demonstrated by considering a simple nonlinear scalar system:

$$
\begin{aligned}
& \dot{x}_{1}=f\left(x_{1}\right)+x_{2} \\
& \dot{x}_{2}=u \\
& y=x_{1}
\end{aligned}
$$

where $x_{1} \in \mathfrak{R}, x_{2} \in \mathfrak{R}, y \in \Re$ and $u \in \mathfrak{R}$. The second equation represent a pure integrator, see Figure 1. Let the design objective be regulation of $y(t) \rightarrow 0$ as $t \rightarrow \infty$. The only 
equilibrium point with $y=0$ is $\left(x_{1}, x_{2}\right)=(0,-f(0))$ corresponding to $\dot{x}_{1}=f\left(x_{1}\right)+x_{2}=0$. The design objective is to render the equilibrium point GAS or GES. Since the nonlinear system (1)-(2) consists of two states $x_{1}$ and $x_{2}$, this will be a recursive design in 2 steps. We will therefore treat (1)-(2) as two cascaded systems each with a single input and output. We start our recursive design with the system $x_{\text {I }}$ and continues with $x_{2}$. We will introduce a change of coordinates during the recursive design process given by:

$$
\mathbf{z}=\phi(\mathbf{x})
$$

where $\mathbf{z}=\left[z_{1}, z_{2}\right]^{T}, \mathbf{x}=\left[x_{1}, x_{2}\right]^{T}$ and $\phi(\mathbf{x}): \mathfrak{R}^{n} \rightarrow \mathfrak{R}^{n}$ is a transformation to be interpreted later. The backstepping transformation is a global diffemorphism, if the mapping $\phi(\mathbf{x})$ is on $\mathfrak{R}^{n}$ with continuously differentiable functions $\phi(\mathbf{x})$ and $\phi^{-1}(\mathbf{x})$, and local if the inverse transformation:

$$
\mathbf{x}=\phi^{-1}(\mathbf{z})
$$

only exists on a subspace of $\mathfrak{R}^{n}$.

\section{Step 1}

For the first system (1) we choose the state $x_{2}$ as a virtual control input while we recall that our design objective is to regulate the output $y=x_{1}$ to zero. Hence, the first backstepping variable is chosen as:

$$
z_{1}=x_{1}
$$

For the system (1) we will treat $x_{2}$ as a virtual control input. The virtual control is defined as:

$$
x_{2} \triangleq \alpha_{1}+z_{2}
$$

where

$$
\begin{aligned}
& \alpha_{1}=\text { stabilizing function } \\
& z_{2}=\text { new state variable }
\end{aligned}
$$

Hence, the $z_{1}$-system can be written:

$$
\dot{z}_{1}=f\left(x_{1}\right)+\alpha_{1}+z_{2}
$$

The new state variable $z_{2}$ will not be used in the first step, but its presence is important since $z_{2}$ is needed to couple the $z_{1}$-system to the next system, that is the $z_{2}$-system to be considered in the next step. Moreover, integrator backstepping implies that the coordinates during the recursive design is changed from $\left(x_{1}, x_{2}\right)$ to $\left(z_{1}, z_{2}\right)$.

We now turn our attention to the design of the stabilizing function $\alpha_{1}$ which will provide the necessary feedback for the $z_{1}$-system. For instance, choosing the stabilizing function as (feedback linearizing control):

$$
\alpha_{1}=-f\left(x_{1}\right)-k_{1} z_{1}
$$

yields:

$$
\dot{z}_{1}=-k_{1} z_{1}+z_{2}
$$

A block diagram showing the stabilizing function and the new state variable is shown in Figurc 2. A Lyapunov function candidate for the $z_{1}$-system is: 


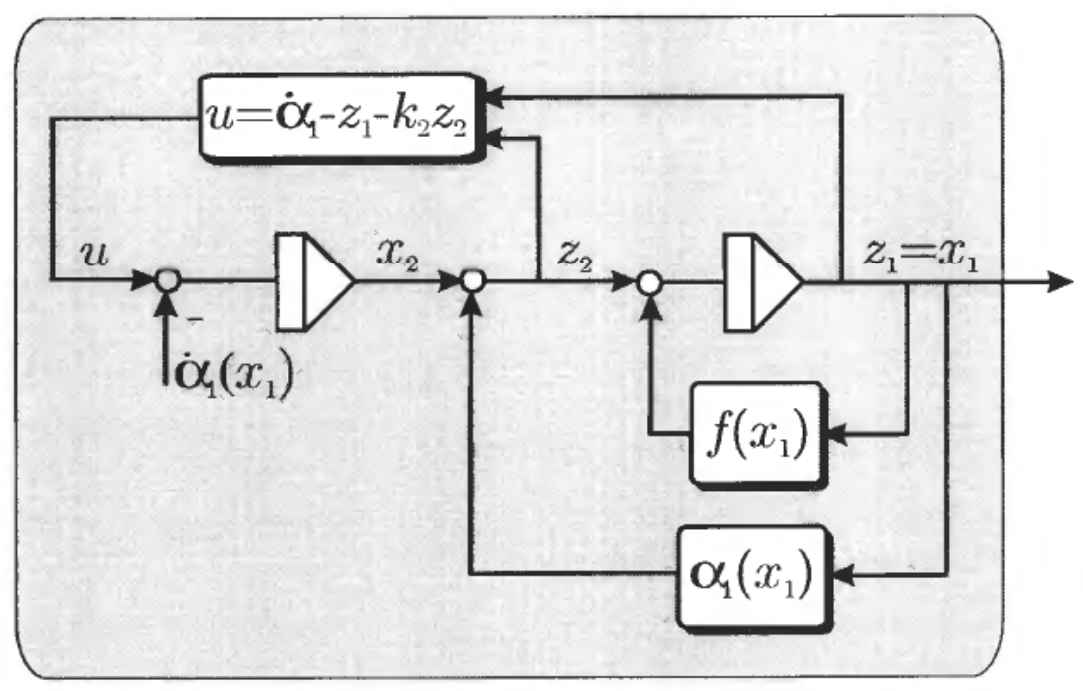

Figure 2. Stabilization of the $x_{1}$-system by means of the stabilizing function $\alpha_{1}=\alpha_{1}\left(x_{1}\right)$. Note that $\dot{\alpha}_{1}\left(x_{1}\right)$ when integrated cancels out the feedback term $-\alpha_{1}\left(x_{1}\right)$.

$$
\begin{aligned}
V_{1} & =\frac{1}{2} z_{1}^{2} \\
\dot{V}_{1} & =z_{1} \dot{z}_{1} \\
& =-k_{1} z_{1}^{2}+z_{1} z_{2}
\end{aligned}
$$

where $k_{1}>0$ is the feedback gain. Hence, the $z_{1}$-system is stabilized. We now turn our attention to the $z_{2}$-system.

\section{Step 2}

The $z_{2}$ dynamics is computed by time differentiation of (5):

$$
\begin{aligned}
\dot{z}_{2} & =\dot{x}_{2}-\dot{\alpha}_{1} \\
& =\boldsymbol{u}-\dot{\alpha}_{1}
\end{aligned}
$$

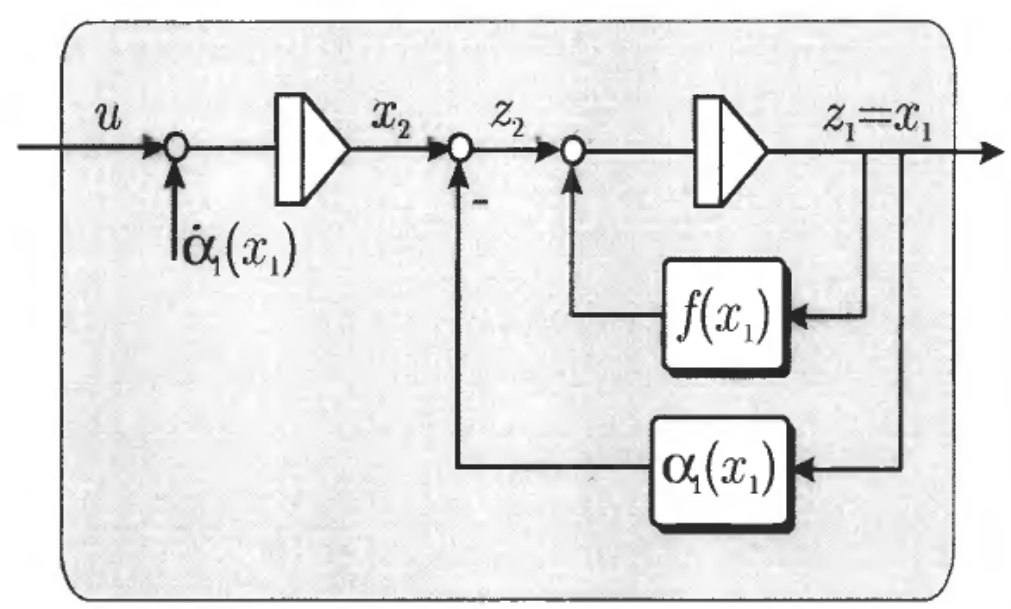

Figure 3. Stabilization of the $x_{2}$-system by means of the control input $u=u\left(\dot{\alpha}_{1}, z_{1}, z_{2}\right)$. 
A Lyapunov function candidate for the $z_{2}$-system is:

$$
\begin{aligned}
V_{2} & =V_{1}+\frac{1}{2} z_{2}^{2} \\
\dot{V}_{2} & =\dot{V}_{1}+\dot{z}_{2} z_{2} \\
& =\left(-k_{1} z_{1}^{2}+z_{1} z_{2}\right)+\dot{z}_{2} z_{2} \\
& =-k_{1} z_{1}^{2}+z_{2}\left(z_{1}+\dot{z}_{2}\right) \\
& =-k_{1} z_{1}^{2}+z_{2}\left(u-\dot{\alpha}_{1}+z_{1}\right)
\end{aligned}
$$

Since our system is described by only two states the control input $u$ appears in the second step. Hence, choosing the control law as:

$$
u=\dot{\alpha}_{1}-z_{1}-k_{2} z_{2}
$$

with $k_{2}>0$, yields:

$$
\dot{V}_{2}=-k_{1} z_{1}^{2}-k_{2} z_{2}^{2}<0, \forall z_{1} \neq 0, z_{2} \neq 0
$$

\section{Implementation aspects}

When implementing the control law (16) it is important to avoid expressions involving the time derivatives of the states. For this simple system only $\dot{\alpha}_{1}$ must be evaluated. This can be done by time differentiation of $\alpha_{1}\left(x_{1}\right)$ along the trajectories of the states. Moreover, we can compute $\dot{\alpha}_{1}$ without using the statc derivatives:

$$
\begin{aligned}
\dot{\alpha}_{1} & =-\frac{\partial f\left(x_{1}\right)}{\partial x_{1}} \dot{x}_{1}-k_{1} \dot{x}_{1} \\
& =-\left(\frac{\partial f\left(x_{1}\right)}{\partial x_{1}}+k_{1}\right)\left(f\left(x_{1}\right)+x_{2}\right)
\end{aligned}
$$

Hence, the final expression for the control law is:

$$
u=-\left(\frac{\partial f\left(x_{1}\right)}{\partial x_{1}}+k_{1}\right)\left(f\left(x_{1}\right)+x_{2}\right)-x_{1}-k_{2}\left(x_{2}+f\left(x_{1}\right)+k_{1} x_{1}\right)
$$

If $f\left(x_{1}\right)=-x_{1}$ (linear theory), we simply get:

$$
\begin{aligned}
u & =-\left(-1+k_{1}\right)\left(-x_{1}+x_{2}\right)-x_{1}-k_{2}\left(x_{2}-x_{1}+k_{1} x_{1}\right) \\
& =-\left(2+k_{1} k_{2}-k_{1}-k_{2}\right) x_{1}-\left(k_{1}+k_{2}-1\right) x_{2}
\end{aligned}
$$

which is a standard PD-control law. The general expression for $u$ is, however, a nonlinear feedback control law depending on the nonlinear function $f\left(x_{1}\right)$.

\section{Backstepping coordinate transformation}

The backstepping coordinate transformation $\mathbf{z}=\phi(\mathbf{x})$ takes the form:

$$
\left[\begin{array}{l}
z_{1} \\
z_{2}
\end{array}\right]=\left[\begin{array}{l}
x_{1} \\
x_{2}-f\left(x_{1}\right)-k_{1} x_{1}
\end{array}\right]
$$

while the inverse transformation $\mathbf{x}=\phi^{-1}(\mathbf{z})$ is:

$$
\left[\begin{array}{l}
x_{1} \\
x_{2}
\end{array}\right]=\left[\begin{array}{l}
z_{1} \\
z_{2}-f\left(z_{1}\right)-k_{1} z_{1}
\end{array}\right]
$$




\section{The Final Check}

If you have performed the backstepping design procedure correctly the dynamics of the closed-loop system in $\left(z_{1}, z_{2}\right)$ coordinates can always be written as the sum of a diagonal and skew-symmetric matrix times the state vector. This can be seen by writing the resulting dynamics in the form:

$$
\left[\begin{array}{l}
\dot{z}_{1} \\
\dot{z}_{2}
\end{array}\right]=\underbrace{-\left[\begin{array}{cc}
k_{1} & 0 \\
0 & k_{2}
\end{array}\right]}_{\text {diagonal matrix }}\left[\begin{array}{l}
z_{1} \\
z_{2}
\end{array}\right]+\underbrace{\left[\begin{array}{cc}
0 & 1 \\
-1 & 0
\end{array}\right]}_{\text {skew-symmetrical matrix }}\left[\begin{array}{l}
z_{1} \\
z_{2}
\end{array}\right]
$$

or equivalently

$$
\dot{\mathbf{z}}=-\mathbf{K z}+\mathbf{S z}
$$

where $\mathbf{z}=\left[z_{1}, z_{2}\right]^{T}, \mathbf{K}=\operatorname{diag}\left\{k_{1}, k_{2}\right\}>0$ and:

$$
\mathbf{S}=-\mathbf{S}^{T}=\left[\begin{array}{cc}
0 & 1 \\
-1 & 0
\end{array}\right]
$$

where $\mathbf{S}$ satisfies $\mathbf{z}^{T} \mathbf{S z}=\mathbf{0}, \forall \mathbf{z}$. In some cases the diagonal matrix will be a function of the state, that is $\mathbf{K}(\mathbf{z})>0$. This is the case when nonlinear damping is added or when some of the nonlinearities are not cancelled by the controller.

\section{Investigation of stability}

We also notice that:

$$
\begin{aligned}
V_{2} & =\frac{1}{2} \mathbf{z}^{T} \mathbf{z} \\
\dot{V}_{2} & =\mathbf{z}^{T}(-\mathbf{K z}+\mathbf{S z}) \\
& =-\mathbf{z}^{T} \mathbf{K} \mathbf{z}
\end{aligned}
$$

Hence, Lyapunov's direct method for autonomous systems ensures that the equilibrium point $\left(x_{1}, x_{2}\right)=(0,-f(0))$ is GAS. In fact, this system will also be GES since it can be shown that the state vector $\mathbf{x}(t)$ decays exponentially to zero by using Theorem 5 in Appendix A, that is:

$$
\|\mathbf{x}(t)\|_{2} \leq e^{-\frac{1}{2} \beta\left(t-t_{0}\right)}\left\|\mathbf{x}\left(t_{0}\right)\right\|_{2}
$$

where $\beta=\lambda_{\min }(\mathbf{K})>0$ is the convergence rate.

A generalization to a large class of lower triangular systems is done in the next chapter.

\subsubsection{Backstepping versus feedback linearization}

The backstepping control law of the previous section is in fact equal to a feedback linearizing controller since the nonlinear function $f\left(x_{1}\right)$ is perfectly compensated for by choosing the stabilizing function as:

$$
\alpha_{1}=-f\left(x_{1}\right)-k_{1} z_{1}
$$

The disadvantage with this approach is that a perfect model of $f\left(x_{1}\right)$ is required. This is impossible in practise. Consequently, an approach cancelling all the nonlinearities may be sensitive for modelling errors. 


\section{Exploiting "good" nonlinearities}

One of the nice features of backstepping is that the stabilizing functions can be modified to exploit so-called "good" nonlinearities. For instance, assume that:

$$
f\left(x_{1}\right)=-\alpha_{0} x_{1}-a_{1} x_{1}^{2}-a_{2}\left|x_{1}\right| x_{1}
$$

where $a_{0}, a_{1}$ and $a_{2}$ are assumed to be unknown positive constants. Since both $a_{0} x_{1}$ and $a_{2}\left|x_{1}\right| x_{1}$ tend to damp out the motion these two expressions should be exploited in the control design and therefore not cancelled out. This can be done by choosing:

$$
\alpha_{1}=a_{1} x_{1}^{2}-k_{1} z_{1}
$$

which only cancels out the destabilizing term $a_{1} x_{1}^{2}$. Unfortunately, this approach is sensitive for modelling errors since $a_{1}$ is not perfectly known. Hence, a better approach would be to choose an expression for $\alpha_{1}$ not depending on $a_{1} x_{1}^{2}$. This can be done by adding nonlinear damping such that the term $a_{1} x_{1}^{2}$ is dominated.

\section{Domination of destabilizing terms by adding nonlinear damping}

The destabilizing term $a_{1} x_{1}^{2}$ can be dominated by adding a nonlinear damping term proportional to $x_{1}^{3}$. Moreover:

$$
\alpha_{1}=-\left[k_{1}+n_{1}\left(z_{1}\right)\right] z_{1}
$$

where $k_{1}>0$ and

$$
n_{1}\left(z_{1}\right)=\kappa_{1} z_{1}^{2} \geq 0
$$

This is shown in Figure 4. Hence:

$$
\begin{aligned}
\dot{z}_{1} & =f\left(z_{1}\right)+\left(\alpha_{1}+z_{2}\right) \\
& =-a_{0} z_{1}-a_{1} z_{1}^{2}-a_{2}\left|z_{1}\right| z_{1}-\left(k_{1}+\kappa_{1} z_{1}^{2}\right) z_{1}+z_{2} \\
& =-\left(a_{0}+a_{2}\left|z_{1}\right|+k_{1}\right) z_{1}-a_{1} z_{1}^{2}-\kappa_{1} z_{1}^{3}+z_{2}
\end{aligned}
$$

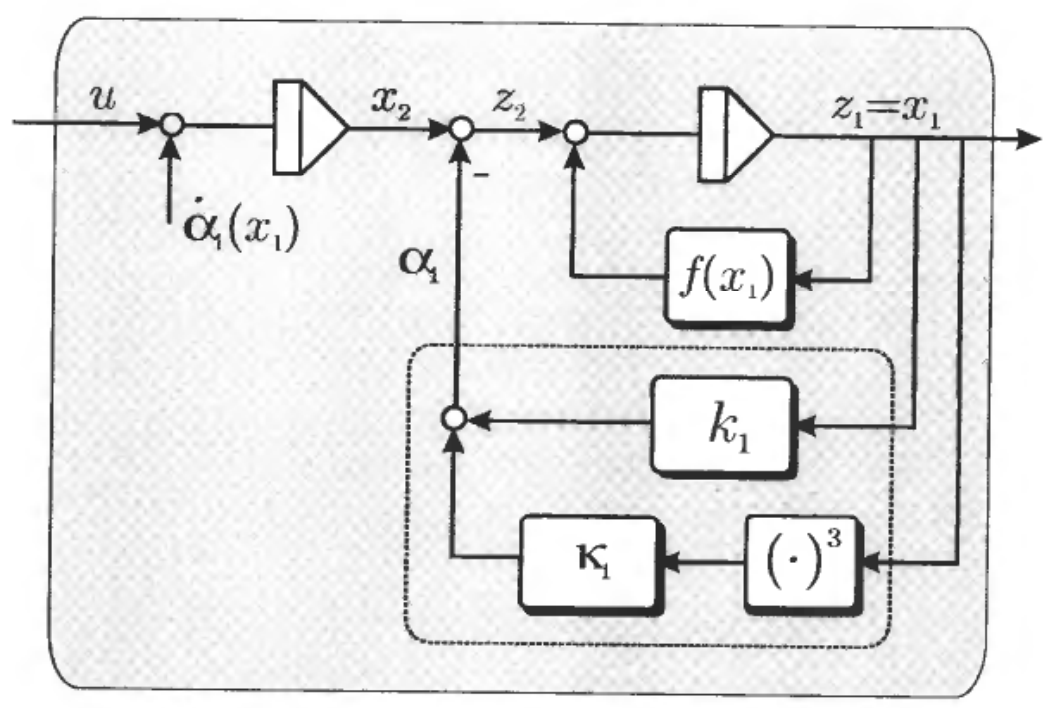

Figure 4. Domination of destabilizing terms by adding nonlinear damping. 
Consider the Lyapunov function:

$$
\begin{aligned}
& V_{1}=\frac{1}{2} z_{1}^{2} \\
& \dot{V}_{1}=-\left(a_{0}+a_{2}\left|z_{1}\right|+k_{1}\right) z_{1}^{2}-a_{1} z_{1}^{3}-\kappa_{1} z_{1}^{4}+z_{1} z_{2}
\end{aligned}
$$

Next:

$$
\begin{aligned}
& V_{2}=V+\frac{1}{2} z_{2}^{2} \\
& \dot{V}_{2}=-\left(a_{0}+a_{2}\left|z_{1}\right|+k_{1}\right) z_{1}^{2}-a_{1} z_{1}^{3}-\kappa_{1} z_{1}^{4}+z_{2}\left(z_{1}+u-\dot{\alpha}_{1}\right)
\end{aligned}
$$

Choosing:

$$
u=\dot{\alpha}_{1}-k_{2} z_{2}-z_{1}
$$

finally yields:

$$
\dot{V}_{2}=-\left(a_{0}+a_{2}\left|z_{1}\right|+k_{1}\right) z_{1}^{2}-a_{1} z_{1}^{3}-\kappa_{1} z_{1}^{4}-k_{2} z_{2}^{3}
$$

This expression can be rewritten by completion of the squares. Moreover:

$$
\begin{aligned}
\dot{V}_{2} & =-\left(\frac{a_{1}}{2 \sqrt{\kappa_{1}}} z_{1}+\sqrt{\kappa_{1}} z_{1}^{2}\right)^{2}+\frac{a_{1}^{2}}{4 \kappa_{1}} z_{1}^{2}-\left(a_{0}+a_{2}\left|z_{1}\right|+k_{1}\right) z_{1}^{2}-k_{2} z_{2}^{2} \\
& \leq \frac{a_{1}^{2}}{4 \kappa_{1}} z_{1}^{2}-\left(a_{0}+a_{2}\left|z_{1}\right|+k_{1}\right) z_{1}^{2}-k_{2} z_{2}^{2} \\
& \leq-\left(a_{0}+k_{1}-\frac{a_{1}^{2}}{4 \kappa_{1}}\right) z_{1}^{2}-k_{2} z_{2}^{2}
\end{aligned}
$$

Hence, by choosing the controller gains according to:

$$
\begin{aligned}
& \kappa_{1}>0 \\
& k_{1}>\frac{a_{1}^{2}}{4 \kappa_{1}}-a_{0} \\
& k_{2}>0
\end{aligned}
$$

our design goal to render $\dot{V}_{2} \leq 0$ is satisfied. Notice that the controller (39) with (32) does not require any information of the unknown parameters $a_{0}, a_{1}$ and $a_{2}$. Hence, a robust nonlinear controller is derived by using backstepping. This result is more attractive than the one obtained from feedback linearization (perfect model cancellation) if robustness is the issue.

\section{Nonlinear backstepping}

\subsection{SISO backstepping}

In order to write the backstepping control laws in a compact notation we will adopt the notation of the Norwegian mathematician, Marius Sophus Lie (1842-1899), who introduced the so-called Lie derivatives in his work.

Definition 1 (Lie Derivative) Let $h: \mathfrak{R}^{n} \rightarrow \mathfrak{R}$ be a smooth scalar function, and $\mathbf{f}: \Re^{n} \rightarrow \mathfrak{R}^{n}$ be a smooth vector field on $\Re^{n}, \mathbf{x} \in \mathfrak{R}^{n}$, then the Lie derivative of the function $h(\mathbf{x})$ with respect to the vector field $\mathbf{f}(\mathbf{x})$ is a scalar function defined by:

$$
L_{f} h(\mathbf{x}) \triangleq \frac{\partial h(\mathbf{x})}{\partial \mathbf{x}} \mathbf{f}(\mathbf{x})
$$


This definition is particularly useful when computing the time derivative of $y$ along the trajectories of $\mathbf{x}$ for the nonlinear system:

$$
\begin{aligned}
\dot{\mathbf{x}} & =\mathbf{f}(\mathbf{x})+\mathbf{g}(\mathbf{x}) u \\
y & =h(\mathbf{x})
\end{aligned}
$$

Hence:

$$
\begin{aligned}
\dot{y} & =\frac{\partial h(\mathbf{x})}{\partial \mathbf{x}} \dot{\mathbf{x}} \\
& =\frac{\partial h(\mathbf{x})}{\partial \mathbf{x}}[\mathbf{f}(\mathbf{x})+\mathbf{g}(\mathbf{x}) u] \\
& =L_{f} h(\mathbf{x})+L_{g} h(\mathbf{x}) u
\end{aligned}
$$

We will now introduce a new definition describing a class of SISO triangular system to be used in the forthcoming sections.

Definition 2 (SISO Strict Feedback Form) A nonlinear system:

$$
\begin{aligned}
\dot{\mathbf{x}} & =\mathbf{f}(\mathbf{x})+\mathbf{g}(\mathbf{x}) u \\
y & =\mathbf{h}(\mathbf{x})
\end{aligned}
$$

where $\mathbf{x}=\left[x_{1}, \ldots, x_{n}\right]^{T} \in \mathfrak{R}^{n}, u \in \mathfrak{R}$ and $y \in \mathfrak{R}$ is said to be in SISO strict feedback form if it can be written in lower triangular form, see Figure 5 .

$$
\begin{aligned}
\dot{x}_{1}= & f_{1}\left(x_{1}\right)+g_{1}\left(x_{1}\right) x_{2} \\
\dot{x}_{2}= & f_{2}\left(x_{1}, x_{2}\right)+g_{2}\left(x_{1}, x_{2}\right) x_{3} \\
\dot{x}_{3}= & f_{3}\left(x_{1}, x_{2}, x_{3}\right)+g_{3}\left(x_{1}, x_{2}, x_{3}\right) x_{4} \\
& \vdots \\
\dot{x}_{n-1}= & f_{n-1}\left(x_{1}, x_{2}, \ldots, x_{n-1}\right)+g_{n-1}\left(x_{1}, x_{2}, \ldots, x_{n-1}\right) x_{n} \\
\dot{x}_{n}= & f_{n}\left(x_{1}, x_{2}, \ldots, x_{k}\right)+g_{n}\left(x_{1}, x_{2}, \ldots, x_{n}\right) u \\
y= & h\left(x_{1}\right)
\end{aligned}
$$

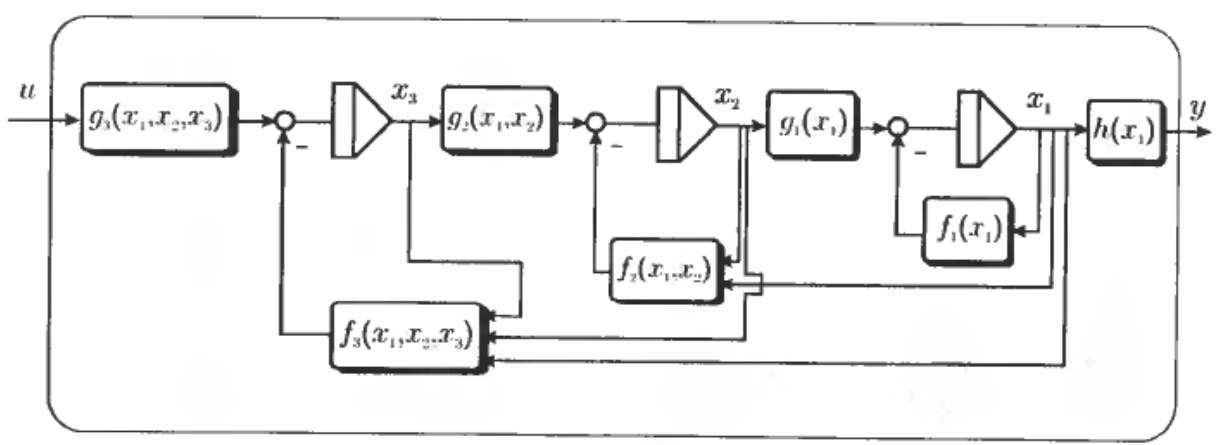

Figure 5. Block diagram showing a 3rd-order nonlinear system in SISO strict feedback form. 
This model class can also be used for tracking by replacing (52) with:

$$
\dot{e}=L_{f 1} h\left(x_{1}\right)+L_{g 1} h\left(x_{1}\right) x_{2}-\dot{y}_{d}
$$

where $e=y-y_{d}$ is the tracking error and $y_{d}(t) \in C^{r}$ is an $r$ times differentable (smooth) and bounded reference trajectory. Regulation of y to zero is obtained by choosing $\dot{y}_{d}=y_{d}=0$ such that $\dot{y}=L_{f 1} h\left(x_{1}\right)+L_{g 1} h\left(x_{1}\right) x_{2}$. In many cases $y=x_{1}$ which can be obtained by choosing $h\left(x_{1}\right)=x_{1}$. Further, it is assumed that $L_{g 1} h\left(x_{1}\right) \neq 0, \forall x_{1}$ and $g_{i}\left(x_{1}, x_{2}, \ldots, x_{i}\right) \neq 0, \forall \mathbf{x}$ for $(i=2 \ldots n)$.

Notice that strict feedback systems are assumed to be affine (linear) in the states $x_{i}$ and the input $u$. The system (52)-(57) is said to be a strict feedback system since the nonlinearity $f_{i}\left(x_{1}, \ldots, x_{i}\right)$ is "fed back" in the $\dot{x}_{i}$-equation, see Figure 5.

\section{Linear systems}

Linearization of (52)-(57) yields a linear system:

$$
\begin{aligned}
\dot{\mathbf{x}} & =\mathbf{A} \mathbf{x}+\mathbf{b} u \\
y & =\mathbf{c}^{T} \mathbf{x}
\end{aligned}
$$

where the structure of $\mathbf{A}$ is lower triangular. Moreover:

$$
\begin{aligned}
\mathbf{A} & =\left[\begin{array}{cccccc}
a_{11} & b_{1} & 0 & \ldots & 0 & 0 \\
a_{21} & a_{22} & b_{2} & \ldots & 0 & 0 \\
a_{31} & a_{32} & a_{33} & \ldots & 0 & 0 \\
\vdots & \vdots & \vdots & \ddots & \vdots & \vdots \\
a_{n-1,1} & a_{n-1,2} & a_{n-1,3} & \ldots & a_{n-1, n-1} & b_{n-1} \\
a_{n, 1} & a_{n, 2} & a_{n, 3} & \ldots & a_{n, n-1} & a_{n, n}
\end{array}\right] \\
\mathbf{b} & =\left[0,0,0, \ldots, 0, b_{n}\right]^{T} \\
\mathbf{c}^{T} & =[1,0,0, \ldots, 0,0]
\end{aligned}
$$

where

$$
\begin{gathered}
a_{i j}=\left.\frac{\partial f_{i}\left(x_{1}, \ldots, x_{i}\right)}{\partial x_{j}}\right|_{x_{i o}} \\
b_{i}=\left.\frac{\partial g_{i}\left(x_{1}, \ldots, x_{i}\right)}{\partial x_{j}}\right|_{x_{i o}}
\end{gathered}
$$

for $(i, j=1 \ldots n)$.

Example 1 (Transfer functions and SISO strict feedback form) Consider the transfer function:

$$
h(s)=\frac{4}{s^{3}+2 s^{2}+4 s+5}
$$

A state-space realization is found by using the Matlab command: [A, B, C, $D]=t f 2 s s\left(4,\left[\begin{array}{lll}1 & 2 & 4\end{array}\right]\right)$. This results in:

$$
\mathbf{A}=\left[\begin{array}{ccc}
-2 & -4 & -5 \\
1 & 0 & 0 \\
0 & 1 & 0
\end{array}\right], \mathbf{b}=\left[\begin{array}{l}
1 \\
0 \\
0
\end{array}\right], \mathbf{c}^{T}=\left[\begin{array}{lll}
0 & 0 & 4
\end{array}\right]
$$


Changing the order of the states $x_{i}$ from 1-2-3 to 3-2-1 yields:

$$
\mathbf{A}=\left[\begin{array}{ccc}
0 & 1 & 0 \\
0 & 0 & 1 \\
-2 & -4 & -5
\end{array}\right], \mathbf{b}=\left[\begin{array}{l}
0 \\
0 \\
1
\end{array}\right], \mathbf{c}^{T}=\left[\begin{array}{lll}
4 & 0 & 0
\end{array}\right]
$$

Hence, this system is in SISO strict feedback form.

A n-th order SISO backstepping control law for nonlinear systems in strict feedback form is stated below.

Theorem 1 (n-th order SISO backstepping control law) The n-th order SISO backstepping controller:

$$
\begin{aligned}
\alpha_{1} & =\frac{1}{L_{g 1} h\left(x_{1}\right)}\left[\dot{y}_{d}-L_{f 1} h\left(x_{1}\right)-k_{1} z_{1}-n_{1}\left(z_{1}\right) z_{1}\right] \\
\alpha_{2} & =\frac{1}{g_{2}\left(x_{1}, x_{2}\right)}\left[\dot{\alpha}_{1}-f_{2}\left(x_{1}, x_{2}\right)-L_{g 1} h\left(x_{1}\right) z_{1}-k_{2} z_{2}-n_{2}\left(z_{2}\right) z_{2}\right] \\
& \vdots \\
(3 \leq i<n) \alpha_{i} & =\frac{1}{g_{i}\left(x_{1}, \ldots, x_{i}\right)}\left[\dot{\alpha}_{i-1}-f_{i}\left(x_{1}, \ldots, x_{i}\right)-g_{i-1}\left(x_{1}, \ldots, x_{i-1}\right) z_{i-1}\right. \\
& \left.-k_{i} z_{i}-n_{i}\left(z_{i}\right) z_{i}\right] \\
\vdots & \\
\alpha_{n} & =\frac{1}{g_{n}\left(x_{1}, \ldots, x_{n}\right)}\left[\dot{\alpha}_{n-1}-f_{n}\left(x_{1}, \ldots, x_{n}\right)-g_{n-1}\left(x_{1}, \ldots, x_{n-2}\right)_{z n-1}\right. \\
& \left.-k_{n} z_{n}-n_{n}\left(z_{n}\right) z_{n}\right] \\
u & =\alpha_{n}
\end{aligned}
$$

where $z_{1}=h\left(x_{1}\right)-y_{d}, z_{i}=x_{i}-\alpha_{i-1}(i=2 \ldots n)$ and the stabilizing functions $\alpha_{i}$ are chosen such that all nonlinearities are cancelled, yields a GES equilibrium point $\mathbf{z}=\mathbf{0}$ for the system ((52)-(56). The controller gains must be chosen such that $k_{i}>0$ and $n_{i}\left(z_{i}\right) \geq 0$ for $(i=1 \ldots n)$.

Proof. The proof follows by induction for $i=1, i=2$ to $i=n$.

\section{Error dynamics}

The resulting error dynamics is written:

$$
\dot{\mathbf{z}}=-\mathbf{K}(\mathbf{z}) \mathbf{z}+\mathbf{S}(\mathbf{x}) \mathbf{z}
$$

where $\mathbf{z}=\left[z_{1}, \ldots, z_{n}\right]^{T}$ and

$$
\mathbf{K}(\mathbf{z})=\operatorname{diag}\left\{k_{1}+n_{1}\left(z_{1}\right), k_{2}+n_{2}\left(z_{2}\right), \ldots, k_{n}+n_{n}(z)\right\}
$$

$$
\mathbf{S}(\mathbf{x})=\left[\begin{array}{ccccccc}
0 & L_{g 1} h\left(x_{1}\right) & 0 & \ldots & 0 & 0 & 0 \\
L_{g} h\left(x_{1}\right) & 0 & g_{2}\left(x_{1}, x_{2}\right) & \ldots & 0 & 0 & 0 \\
0 & -g_{2}\left(x_{1}, x_{2}\right) & 0 & \ldots & 0 & 0 & 0 \\
\vdots & \vdots & \vdots & \ddots & \vdots & \vdots & \vdots \\
0 & 0 & 0 & \ldots & 0 & g_{n-1}\left(x_{1}, \ldots, x_{n-1}\right) & 0 \\
0 & 0 & 0 & \ldots & g_{n-1}\left(x_{1}, \ldots, x_{n-1}\right) & -g_{n}\left(x_{1}, \ldots, x_{n}\right) & g_{n}\left(x_{1}, \ldots, x_{n}\right) \\
0 & 0 & 0 & \ldots & 0 & 0
\end{array}\right]
$$

Hence, the equilibrium point $\mathbf{z}=0$ is GES since $\mathbf{z}^{T} \mathbf{S}(\mathbf{x}) \mathbf{z}=0, \forall \mathbf{x}, \mathbf{z}$ and $\mathbf{K}(\mathbf{z})$ and $\mathbf{K}(\mathbf{z})>0, \forall \mathbf{z}$, implies that: 


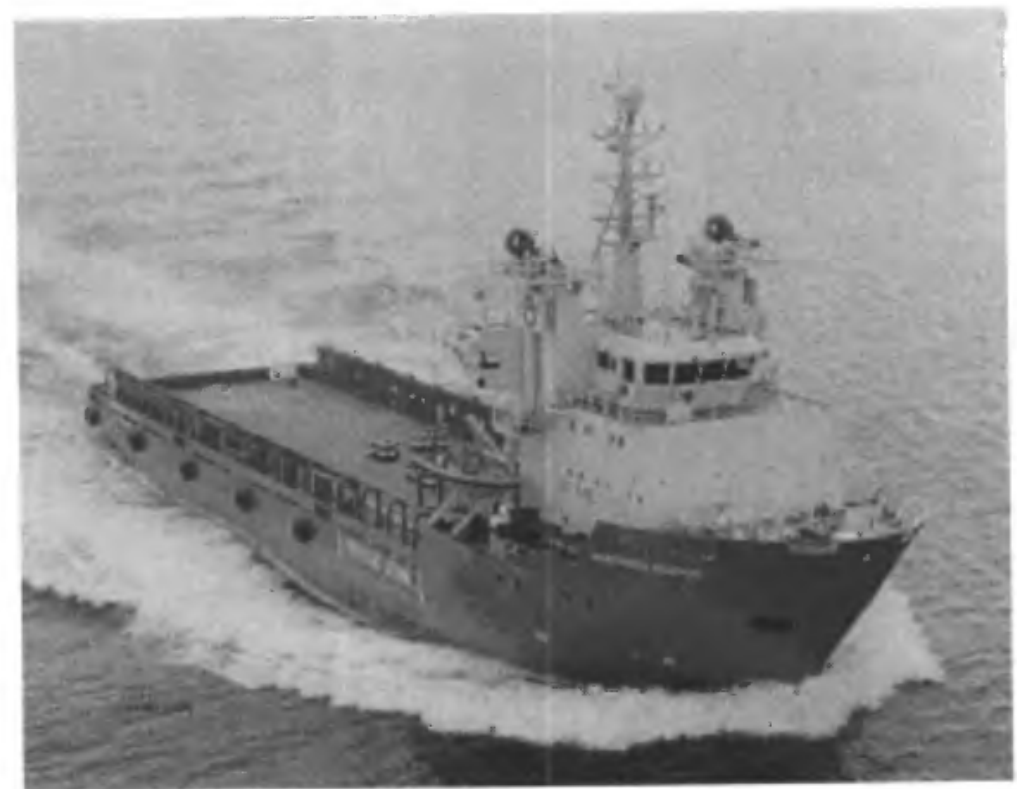

Figure 6. Supply vessel used in the North Sea. Length of ship: $76 \cdot 2 \mathrm{~m}$.

$$
\begin{aligned}
& V_{n}(\mathbf{z})=\frac{1}{2} \mathbf{z}^{T} \mathbf{z} \\
& \dot{V}_{n}(\mathbf{z})=-\mathbf{z}^{T} \mathbf{K}(\mathbf{z}) \mathbf{z}
\end{aligned}
$$

Notice that if the output mapping $y=h\left(x_{1}\right)$ is chosen as $y=x_{1}$, the element $S_{12}=-S_{21}=L_{g_{1}} h\left(x_{1}\right)$ reduces to $S_{12}=-S_{21}=g_{1}\left(x_{1}\right)$ which is consistent with the other non-zero off-diagonal elements of $\mathbf{S}$.

\subsubsection{Nonlinear ship autopilot design}

The yaw dynamics of a rudder controlled ship is described by the Nomoto model (Fossen, 1994):

$$
\begin{aligned}
\frac{\Psi}{\delta}(s) & =\frac{K\left(1+T_{3} s\right)}{s\left(1+T_{1} s\right)\left(1+T_{2} s\right)} \\
& \approx \frac{K}{s(1+T s)}
\end{aligned}
$$

where $T_{1}, T_{2}, T_{3}$ and $K$ are the Nomoto time and gain constants, $T=T_{1}+T_{2}-T_{3}$ is the equivalent time constant, $\Psi$ is the yaw angle (heading of the ship and $\delta$ is the rudder angle. This model is often extended to include nonlinear maneuvering characteristics in order to describe both course-stable and course-unstable ships more accurate. We will show how backstepping can be applied to both the 2nd and 3rd order models of Nomoto.

The nonlinear model of Norrbin (1963)

Consider the nonlinear ship model:

$$
T \ddot{\Psi}+H_{N}(\dot{\Psi})=K \delta
$$




$$
H_{N}(\dot{\Psi})=n_{3} \dot{\Psi}^{3}+n_{2} \dot{\Psi}^{2}+n_{1} \dot{\Psi}+n_{0}
$$

where $H_{N}(\dot{\Psi})$ is the nonlinear maneuvering characteristic. For a course-unstable ship $n_{1}<0$ whereas course-stable ship satisfies $n_{1}>0$. For single-screwed ships (one propeller) $n_{0} \neq 0$. Similarly, symmetry in the hull implies that $n_{2}=0$. The bias term $n_{0}$ is usually compensated for by including integral action in the autopilot since this term is impossible to compute due to an unknown component caused by the environmental disturbances (wind, waves and currents). Hence, we propose to use the polynominal:

$$
H_{N}(\dot{\Psi})=n_{3} \dot{\Psi}^{3}+n_{1} \dot{\Psi}
$$

in our design. Also notice that the term $n_{3} \dot{\Psi}^{3}$ is " good" damping since the ship-ambient water system is dissipative while the linear term $n_{1} \dot{\Psi}$ is destabilizing for courseunstable ships. This is usually the case for large tankers whereas smaller ships are course-stable. However, feedback from $\dot{\Psi}$ will stabilize course-unstable ships. We will illustrate how the SISO backstepping control law can be derived by solving this problem in 2 steps:

\section{Step 1:}

Let the control objective be tracking of $e=\Psi-\Psi_{d}$ to zero. Hence:

$$
\begin{aligned}
z_{1} & =\boldsymbol{e} \\
\dot{z}_{1} & =\dot{\Psi}-\dot{\Psi}_{d} \\
& =\boldsymbol{r}-\dot{\Psi}_{d}
\end{aligned}
$$

Taking $r$ as virtual control:

$$
r \bumpeq \alpha_{1}+z_{2}
$$

where $z_{2}$ is a new state variable to be interpreted later, yields:

$$
\dot{z}_{1}=\alpha_{1}+z_{2}-\dot{\Psi}_{d}
$$

Next, we choose the stabilizing function $\alpha_{1}$ such that $z_{1} \rightarrow 0$. Moreover:

$$
\alpha_{1}=\dot{\Psi}_{d}-k_{1} z_{1}-n_{1}\left(z_{1}\right) z_{1}
$$

yields:

$$
\dot{z}_{1}=-\left[k_{1}+n_{1}\left(z_{1}\right)\right] z_{1}+z_{2}
$$

where $k_{1}>0$ and $n_{1}\left(z_{1}\right) \geq 0$ are design parameters. A Lyapunov function candidate for $z_{1}$ is:

$$
\begin{aligned}
V_{\mathbf{I}} & =\frac{1}{2} z_{1}^{2} \\
\dot{V}_{1} & =z_{1} \dot{z}_{1} \\
& =-\left[k_{1}+n_{1}\left(z_{1}\right)\right] z_{1}^{2}+z_{1} z_{2}
\end{aligned}
$$

Step 2:

The second step stabilizes the $z_{2}$-dynamics:

$$
\dot{z}=\dot{r}-\dot{\alpha}_{1}=\frac{K}{T} \delta-\frac{1}{T} H_{N}(\dot{\Psi})-\dot{\alpha}_{1}
$$


The second Lyapunov function candidate is:

$$
\begin{aligned}
V_{2} & =V_{1}+\frac{1}{2} z_{2}^{2} \\
\dot{V}_{2} & =\dot{V}_{1}+z_{2} \dot{z}_{2} \\
& =-\left[k_{1}+n_{1}\left(z_{1}\right)\right] z_{1}^{2}+z_{2}\left(z_{1}+\dot{z}_{2}\right) \\
& =-\left[k_{1}+n_{1}\left(z_{1}\right)\right] z_{1}^{2}+z_{2}\left[z_{1}+\frac{K}{T} \delta-\frac{1}{T} H_{N}(\dot{\Psi})-\dot{\alpha}_{1}\right]
\end{aligned}
$$

Since the input $\delta$ appears in $\dot{V}_{2}$ we can predescribe a value for $\delta$ such that $\dot{V}_{2}$ becomes negative definite. For instance:

$$
\delta=\frac{T}{K}\left[\dot{\alpha}_{1}-\left[k_{2}+n_{2}\left(z_{2}\right)\right] z_{2}-z_{1}\right]+\frac{1}{K} H_{N}(\dot{\Psi})
$$

where $k_{2}>0$ and $n_{2}\left(z_{2}\right) \geq 0$ are design parameters. Hence:

$$
\begin{aligned}
\dot{V}_{2} & =-\left[k_{1}+n_{1}\left(z_{1}\right)\right] z_{1}^{2}-\left[k_{2}+n_{2}\left(z_{2}\right)\right] z_{2}^{2} \\
& <0, \forall z_{1} \neq 0, z_{2} \neq 0
\end{aligned}
$$

When implementing the control law, $\dot{\alpha}_{1}$ is rewritten as:

$$
\begin{aligned}
\dot{\alpha}_{1} & =\frac{\partial \alpha_{1}}{\partial \dot{\Psi}_{d}} \ddot{\Psi}_{d}+\frac{\partial \alpha_{1}}{\partial z_{1}} \dot{z}_{1} \\
& =\ddot{\Psi}_{d}-\left[k_{1}+\frac{\partial\left[n_{1}\left(z_{1}\right) z_{1}\right]}{\partial z_{1}}\right]\left(\alpha_{1}+z_{2}-\dot{\Psi}_{d}\right)
\end{aligned}
$$

in order to avoid the state derivatives in the control law.

Also notice that, the SISO backstepping control law can be found directly from Theorem 1 with $n=2$, that is:

$$
\begin{aligned}
\alpha_{1} & =\dot{y}_{d}-k_{1} z_{1}-n_{1}\left(z_{1}\right) z_{1} \\
u & =\frac{T}{K}\left(\dot{\alpha}_{1}-z_{1}+\frac{1}{T} H_{N}\left(x_{2}\right)-k_{2} z_{2}-n_{2}\left(z_{2}\right) z_{2}\right)
\end{aligned}
$$

where $y_{d}=\Psi_{d}$ and

$$
\begin{aligned}
& x_{1}=\Psi \\
& x_{2}=r=\dot{\Psi}
\end{aligned}
$$

\section{Resulting error dynamics}

The resulting error dynamics is written:

$$
\begin{aligned}
{\left[\begin{array}{l}
\dot{z}_{1} \\
\dot{z}_{2}
\end{array}\right] } & =-\left[\begin{array}{cc}
k_{1}+n_{1}\left(z_{1}\right) & 0 \\
0 & k_{2}+n_{2}\left(z_{2}\right)
\end{array}\right]\left[\begin{array}{l}
z_{1} \\
z_{2}
\end{array}\right]+\left[\begin{array}{cc}
0 & 1 \\
-1 & 0
\end{array}\right]\left[\begin{array}{l}
z_{1} \\
z_{2}
\end{array}\right] \\
\dot{\mathbf{z}} & =-\mathbf{K}(\mathbf{z}) \mathbf{z}+\mathbf{S} \mathbf{z}
\end{aligned}
$$

Hence, the resulting system is GES since:

$$
\begin{aligned}
& V_{2}(\mathbf{z})=\frac{1}{2} \mathbf{z}^{T} \mathbf{z} \\
& \dot{V}_{2}(\mathbf{z})=\mathbf{z}^{T}[-\mathbf{K}(\mathbf{z})+\mathbf{S}] \mathbf{z}
\end{aligned}
$$




$$
\begin{aligned}
& =-\mathbf{z}^{T} \mathbf{K}(\mathbf{z}) \\
& <0, \forall \mathbf{z} \neq \mathbf{0}
\end{aligned}
$$

where we have used the skew-symmetric property $\mathbf{z T S z}=0, \forall \mathbf{z}$.

We will now extend this result to the 3rd order model.

The nonlinear model of Bech and Wagner Smith (1969)

Consider the nonlinear ship model:

$$
\begin{aligned}
T_{1} T_{2} \Psi^{(3)}+\left(T_{1}+T_{2}\right) \ddot{\Psi}+K H_{B}(\dot{\Psi}) & =K\left(\delta+T_{3} \dot{\delta}\right) \\
H_{B}(\dot{\Psi}) & =b_{3} \dot{\Psi}^{3}+b_{2} \dot{\Psi}^{2}+b_{1} \dot{\Psi}+b_{0}
\end{aligned}
$$

where $H_{B}(\dot{\Psi})$ is the nonlinear maneuvering characteristic.

The 3rd-order yaw dynamics (94) can be written in SISO strict feedback form as:

$$
\begin{aligned}
\dot{x}_{1} & =x_{2} \\
\dot{x}_{2} & =x_{3} \\
\dot{x}_{3} & =f\left(x_{2}\right)+a x_{3}+b u \\
y & =x_{1}
\end{aligned}
$$

where $f\left(x_{2}\right)=-\frac{K}{T_{1} T_{2}} H_{B}\left(x_{2}\right), a=-\frac{T_{1}+T_{2}}{T_{1} T_{2}}, b=\frac{K}{T_{1} T_{2}}$ and:

$$
\begin{aligned}
x_{1} & =\Psi \\
x_{2} & =r=\dot{\Psi} \\
x_{3} & =\dot{r}=\ddot{\Psi} \\
u & =\delta+T_{3} \dot{\delta}
\end{aligned}
$$

The rudder angle $\delta$ is computed by numerical integration of:

$$
\dot{\delta}=-\frac{1}{T_{3}}(\delta-u)
$$

with $u$ given by the backstepping control law (Theorem 1):

$$
\begin{aligned}
\alpha_{1} & =\dot{y}_{d}-k_{1} z_{1}-n_{1}\left(z_{1}\right) z_{1} \\
\alpha_{2} & =\dot{\alpha}_{1}-z_{1}-k_{2} z_{2}-n_{2}\left(z_{2}\right) z_{2} \\
u & =\frac{1}{b}\left(\dot{\alpha}_{2}-z_{2}-f\left(x_{2}\right)-a x_{3}-k_{3} z_{3}-n_{3}\left(z_{3}\right) z_{3}\right)
\end{aligned}
$$

Hence $z_{1}=y-y_{d}$. Feedback from the yaw angle $\Psi$ and yaw rate $r=\dot{\Psi}$ can be realized since these signals are easily measured by a gyro compass and a rate gyro. However, the signal $r$ is not available for feedback unless a state observer is constructed.

\subsection{MIMO vectorial backstepping}

In this chapter we will show how the backstepping control laws for nonlinear systems in SISO strict fecdback form can be gencralized to MIMO systems. 


\subsubsection{Vectorial backstepping}

It is straightforward to extend the results of the previous section by writing the nonlinear system equations in a vectorial setting. In order to do this we will call a vector function $\mathbf{f}: \Re^{n} \rightarrow R^{n}$ a vector field in $\Re^{n}$. Similarly, the stabilizing functions $\alpha_{i}: \Re^{n} \rightarrow \mathfrak{R}$ will now be replaced by stabilizing vector fields $\boldsymbol{\alpha}_{i}: \mathfrak{R}^{n} \rightarrow \mathfrak{R}^{n}$.

Motivated by the definition of the SISO strict feedback form we will now define a model class for MIMO nonlinear systems in lower block triangular form. In order to simplify the notation, we will introduce the Jacobian of a vector field.

Definition 3 (Jacobian) Let $\mathbf{h}: \mathfrak{R}^{n} \rightarrow \mathfrak{R}^{n}$ be a smooth vector field, $\mathbf{x} \in \mathfrak{R}^{n}$, then the Jacobian of $\mathbf{h}(\mathbf{x})$ is denoted by:

$$
\nabla \mathbf{h}(\mathbf{x}) \triangleq \frac{\partial \mathbf{h}(\mathbf{x})}{\partial \mathbf{x}}
$$

The Jacobian is an $n \times n$ matrix of elements $\nabla \mathbf{h}(\mathbf{x})_{i j}=\partial h_{i} / \partial x_{j}$. If $\mathbf{h}: \mathfrak{R}^{n} \rightarrow \mathfrak{R}^{m}(m<n)$, we will refer to $\nabla \mathbf{h}(\mathbf{x})$ as a $m \times n$ matrix of partial derivatives.

Definition 3 together with the SISO results in the previous section motivates the following definition:

Definition 4 (MIMO strict feedback form) A MIMO nonlinear system:

$$
\begin{aligned}
\dot{\mathbf{x}} & =\mathbf{f}(\mathbf{x})+\mathbf{G}(\mathbf{x}) \mathbf{u} \\
\mathbf{y} & =\mathbf{h}(\mathbf{x})
\end{aligned}
$$

where $\mathbf{x}=\left[\mathbf{x}_{1}^{T}, \mathbf{x}_{2}^{T}, \ldots, \mathbf{x}_{n}^{T}\right]^{T} \in \mathfrak{R}^{n}, \mathbf{u} \in \mathfrak{R}^{r}$ and $\mathbf{y} \in \mathfrak{R}^{m}(r \geq m)$ is said to be in MIMO strict feedback form if it can be written in lower block triangular form:

$$
\begin{aligned}
\dot{\mathbf{x}}_{1}= & \mathbf{f}_{1}\left(\mathbf{x}_{1}\right)+\mathbf{G}_{1}\left(\mathbf{x}_{1}\right) \mathbf{x}_{2} \\
\dot{\mathbf{x}}_{2}= & \mathbf{f}_{2}\left(\mathbf{x}_{1}, \mathbf{x}_{2}\right)+\mathbf{G}_{2}\left(\mathbf{x}_{1}, \mathbf{x}_{2}\right) \mathbf{x}_{3} \\
\dot{\mathbf{x}}_{3}= & \mathbf{f}_{3}\left(\mathbf{x}_{1}, \mathbf{x}_{2}, \mathbf{x}_{3}\right)+\mathbf{G}_{3}\left(\mathbf{x}_{1}, \mathbf{x}_{2}, \mathbf{x}_{3}\right) \mathbf{x}_{4} \\
& \vdots \\
\dot{\mathbf{x}}_{n-1}= & \mathbf{f}_{n-1}\left(\mathbf{x}_{1}, \mathbf{x}_{2}, \ldots, \mathbf{x}_{n-1}\right)+\mathbf{G}_{n-1}\left(\mathbf{x}_{1}, \mathbf{x}_{2}, \ldots, \mathbf{x}_{n-1}\right) \mathbf{x}_{n} \\
\dot{\mathbf{x}}_{n}= & \mathbf{f}_{n}\left(\mathbf{x}_{1}, \mathbf{x}_{2}, \ldots, \mathbf{x}_{n}\right)+\mathbf{G}_{n}\left(\mathbf{x}_{1}, \mathbf{x}_{2}, \ldots, \mathbf{x}_{n}\right) \mathbf{u} \\
\mathbf{y}= & \mathbf{h}\left(\mathbf{x}_{1}\right)
\end{aligned}
$$

with:

$$
\operatorname{dim} \mathbf{y} \leq \operatorname{dim} \mathbf{x}_{1} \leq \operatorname{dim} \mathbf{x}_{2} \leq \ldots \leq \operatorname{dim} \mathbf{x}_{n} \leq \operatorname{dim} \mathbf{u}
$$

This model class can be used for tracking control by replacing (111) with:

$$
\dot{\mathbf{e}}=\nabla \mathbf{h}\left(\mathbf{x}_{1}\right) \mathbf{f}_{1}\left(\mathbf{x}_{1}\right)+\nabla \mathbf{h}\left(\mathbf{x}_{1}\right) \mathbf{G}_{1}\left(\mathbf{x}_{1}\right) \mathbf{x}_{2}-\dot{\mathbf{y}}_{d}
$$

where $\mathbf{e}=\mathbf{y}-\mathbf{y}_{d}$ is the tracking error only $\mathbf{y}_{\mathrm{d}}(t)$ is an $r$ times differentiable (smooth) and bounded reference trajectory. Regulation of $\mathbf{y}$ to zero is obtained by choosing $\dot{\mathbf{y}}_{d}=\mathbf{y}_{d}=0$ such that $\mathbf{e}=\mathbf{y}$. It is assumed that both $\nabla \mathbf{h} \mathbf{G}_{1}\left(\nabla \mathbf{h} \mathbf{G}_{1}\right)^{T}$ and $\mathbf{G}_{i} \mathbf{G}_{i}^{T}$ for $(i=2 \ldots n)$ are invertible for all $\mathbf{x}_{i}$ such that

$$
\begin{aligned}
\left(\nabla \mathbf{h} \mathbf{G}_{1}\right)^{\dagger} & =\left(\nabla \mathbf{h} \mathbf{G}_{1}\right)^{T}\left[\left(\nabla \mathbf{h} \mathbf{G}_{1}\right)\left(\nabla \mathbf{h} \mathbf{G}_{1}\right)^{T}\right]^{-1} \\
\mathbf{G}_{i}^{\dagger} & =\mathbf{G}_{i}^{T}\left(\mathbf{G}_{i} \mathbf{G}_{i}^{T}\right)^{-1}
\end{aligned}
$$

exist. 
Example 2 (MIMO strict feedback form) Consider the MIMO nonlinear system:

$$
\begin{aligned}
& \dot{x}_{1}=-x_{1}^{3}+x_{2} \\
& \dot{x}_{2}=-x_{1}-x_{2}+x_{3}+2 u_{1}+u_{2} \\
& \dot{x}_{3}=x_{1}^{2}+x_{2}+x_{3}+u_{2} \\
& y=x_{1}
\end{aligned}
$$

which can be written as two subsystems $\mathbf{x}_{1}=\left[x_{1}\right]$ and $\mathbf{x}_{2}=\left[x_{2}, x_{3}\right]^{T}$ according to:

$$
\begin{aligned}
\dot{x}_{1} & =-x_{1}^{3}+\left[\begin{array}{ll}
1 & 0
\end{array}\right]\left[\begin{array}{l}
x_{2} \\
x_{3}
\end{array}\right] \\
{\left[\begin{array}{c}
\dot{x}_{2} \\
\dot{x}_{3}
\end{array}\right] } & =\left[\begin{array}{c}
-x_{1}-x_{2}+x_{3} \\
x_{1}^{2}+x_{2}+x_{3}
\end{array}\right]+\left[\begin{array}{ll}
2 & 1 \\
0 & 1
\end{array}\right]\left[\begin{array}{l}
u_{1} \\
u_{2}
\end{array}\right] \\
y & =x_{1}
\end{aligned}
$$

Since

$$
\nabla \mathbf{h} \mathbf{G}_{1}\left(\nabla \mathbf{h} \mathbf{G}_{1}\right)^{T}=\mathbf{G}_{1} \mathbf{G}_{1}^{T}=1
$$

and

$$
\operatorname{det}\left(\mathbf{G}_{2} \mathbf{G}_{2}^{T}\right)=\operatorname{det}\left[\begin{array}{ll}
5 & 1 \\
1 & 1
\end{array}\right]=4
$$

this system is in MIMO strict feedback form.

The MIMO recursive design procedure is similar to the SISO case. The main result is stated in the following theorem:

Theorem 2 (MIMO backstepping control law) The MIMO backstepping controller:

$$
\begin{aligned}
& \boldsymbol{\alpha}_{1}=\left(\nabla \mathbf{h}\left(\mathbf{x}_{1}\right) \mathbf{G}_{1}\left(\mathbf{x}_{1}\right)\right)^{\dagger}\left[\dot{\mathbf{y}}_{d}-\nabla \mathbf{h}\left(\mathbf{x}_{1}\right) \mathbf{f}\left(\mathbf{x}_{1}\right)-\mathbf{K}_{1} \mathbf{z}_{1}-\mathbf{N}_{1}\left(\mathbf{z}_{1}\right) \mathbf{z}_{1}\right] \\
& \boldsymbol{\alpha}_{2}=\mathbf{G}_{2}^{\dagger}\left(\mathbf{x}_{1}, \mathbf{x}_{2}\right)\left[\dot{\alpha}_{1}-\mathbf{f}_{2}\left(\mathbf{x}_{1}, \mathbf{x}_{2}\right)-\nabla \mathbf{h}\left(\mathbf{x}_{1}\right) \mathbf{G}_{1}\left(\mathbf{x}_{1}\right) \mathbf{z}_{1}-\mathbf{K}_{2} \mathbf{z}_{2}\right. \\
& \left.-\mathbf{N}_{2}\left(\mathbf{z}_{2}\right) \mathbf{z}_{2}\right] \\
& \vdots \\
& (3 \leq i<n) \alpha_{i}=\mathbf{G}_{i}^{\dagger}\left(\mathbf{x}_{1}, \ldots, \mathbf{x}_{i}\right)\left[\dot{\alpha}_{i-1}-\mathbf{f}_{i}\left(\mathbf{x}_{1}, \ldots, \mathbf{x}_{i}\right)-\mathbf{G}_{i-1}\left(\mathbf{x}_{1}, \ldots, \mathbf{x}_{i-1}\right) \mathbf{z}_{i-1}\right. \\
& \left.-\mathbf{K}_{i} \mathbf{z}_{i}-\mathbf{N}_{i}\left(\mathbf{z}_{i}\right) \mathbf{z}_{i}\right] \\
& \dot{\alpha}_{n}=\mathbf{G}_{n}^{\dagger}\left(\mathbf{x}_{1}, \ldots, \mathbf{x}_{n}\right) \dot{\alpha}_{n-1}-\mathbf{f}_{n}\left(\mathbf{x}_{1}, \ldots, \mathbf{x}_{n}\right)-\mathbf{G}_{n-1}\left(\mathbf{x}_{1}, \ldots, \mathbf{x}_{n-1}\right) \mathbf{z}_{n-1} \\
& \left.-\mathbf{K}_{n} \mathbf{z}_{n}-\mathbf{N}_{n}\left(\mathbf{z}_{n}\right) \mathbf{z}_{n}\right] \\
& \mathbf{u}=\boldsymbol{\alpha}_{n}
\end{aligned}
$$

where $\mathbf{z}_{1}=\mathbf{h}\left(\mathbf{x}_{1}\right)-\mathbf{y}_{d}, \mathbf{z}_{i}-\boldsymbol{\alpha}_{i-1}(i=2 \ldots . n)$ and the stabilizing vector fields $\alpha_{i}$ are chosen such that all nonlinearities are cancelled, yields a GES equilibrium point $\mathbf{z}=\mathbf{0}$ for the system (111)-(115) if the controller gains satisfy $\mathbf{K}_{i}>0$ and $\mathbf{N}_{i}\left(\mathbf{z}_{i}\right) \geq 0$ $(i=1 \ldots n)$.

Proof. The proof follows by induction for $i=1, i=2$ to $i-n$. 


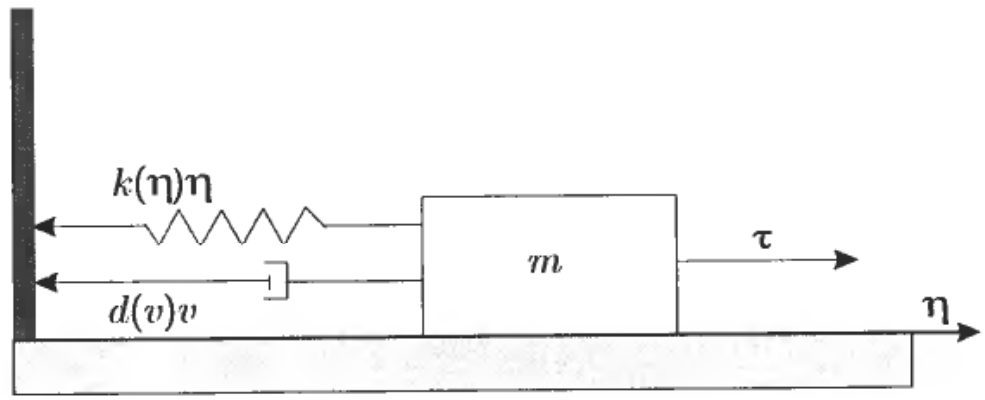

Figure 7. SISO nonlinear mass-damper-spring system.

\section{Error Dynamics}

The resulting error dynamics is written:

$$
\dot{\mathbf{z}}=-\mathbf{K}(\mathbf{z}) \mathbf{z}+\mathbf{S}(\mathbf{x}) \mathbf{z}
$$

where $\mathbf{z}=\left[\mathbf{z}_{1}, \ldots, \mathbf{z}_{i}\right]^{T}$ and

$$
\begin{aligned}
& \mathbf{K}=\left[\begin{array}{cccc}
\mathbf{K}_{1}+\mathbf{N}_{1}\left(\mathbf{z}_{1}\right) & 0 & \ldots & 0 \\
0 & \mathbf{K}_{2}+\mathbf{N}_{2}\left(\mathbf{z}_{2}\right) & \cdots & 0 \\
\vdots & \vdots & \ddots & 0 \\
0 & 0 & 0 & \mathbf{K}_{i}+\mathbf{N}_{i}\left(\mathbf{z}_{i}\right)
\end{array}\right] \\
& \mathbf{S}=\left(\begin{array}{ccccccc}
0 & \boldsymbol{\nabla h}\left(\mathbf{x}_{1}\right) \mathbf{G}_{1}\left(\mathbf{x}_{1}\right) & 0 & \ldots & 0 & 0 & 0 \\
-\nabla \mathbf{h}\left(\mathbf{x}_{1}\right) \mathbf{G},\left(\mathbf{x}_{1}\right) & 0 & \mathbf{G}_{2}\left(\mathbf{x}_{1}, \mathbf{x}_{2}\right) & \ldots & 0 & 0 & 0 \\
0 & -\mathbf{G}_{2}\left(\mathbf{x}_{1}, \mathbf{x}_{2}\right) & 0 & \ldots & 0 & 0 & 0 \\
\vdots & \vdots & \vdots & \ddots & \vdots & \vdots & \vdots \\
0 & 0 & 0 & \ldots & 0 & \mathbf{G}_{i-1}\left(\mathbf{x}_{1}, \ldots, \mathbf{x}_{i-1}\right) & 0 \\
0 & 0 & 0 & \ldots & -\mathbf{G}_{i-1}\left(\mathbf{x}_{1}, \ldots, \mathbf{x}_{i-1}\right) & 0 & \mathbf{G}_{i}\left(\mathbf{x}_{1}, \ldots, \mathbf{x}_{i}\right) \\
0 & 0 & 0 & \ldots & 0 & -\mathbf{G}_{i}\left(\mathbf{x}_{1}, \ldots, \mathbf{x}_{i}\right) & 0
\end{array} \mid\right.
\end{aligned}
$$

\subsubsection{Example: nonlinear mass-damper-spring system}

Consider a MIMO nonlinear mass-damper-spring system in the form:

$$
\begin{aligned}
\dot{\boldsymbol{\eta}} & =\boldsymbol{v} \\
\mathbf{M} \dot{\boldsymbol{v}}+\mathbf{D}(\mathbf{v}) \mathbf{v}+\mathbf{K}(\boldsymbol{\eta}) \boldsymbol{\eta} & =\mathbf{B u}
\end{aligned}
$$

where $\boldsymbol{\eta} \in \mathfrak{R}^{n}$ is the position vector, $\mathbf{v} \in \mathfrak{R}^{n}$ is the velocity vector, $\mathbf{u} \in \mathfrak{R}^{r}(r \geq n)$ is the control input vector, $\mathbf{D}(\mathbf{v}) \in \mathfrak{R}^{n \times n}$ represents a matrix of damping coefficients, $\mathbf{K}(\boldsymbol{\eta}) \in \mathfrak{R}^{n \times n}$ is a matrix of spring stiffness coefficients, $\mathbf{M} \in \mathfrak{R}^{n \times n}$ is the inertia matrix and $\mathbf{B} \in \mathfrak{R}^{n \times r}$ is the input matrix. Hence, backstepping can be performed in two vectorial steps.

\section{Step 1:}

Let $v$ be the virtual control vector:

$$
\mathbf{v} \cong \mathbf{s}+\alpha_{1}
$$


where

$$
\begin{array}{cc}
\mathbf{s}=\tilde{\boldsymbol{v}}+\Lambda \tilde{\boldsymbol{v}} & \text { New state vector used for tracking control } \\
\boldsymbol{\alpha}_{1} & \text { Stabilizing vector field to be defined later }
\end{array}
$$

Here $\tilde{\boldsymbol{v}}=\boldsymbol{v}-\boldsymbol{v}_{d}$ and $\tilde{\boldsymbol{\eta}}=\boldsymbol{\eta}-\boldsymbol{\eta}_{d}$ are the velocity and position tracking errors, respectively and $\Lambda$ is a diagonal matrix of positive elements. The definition of the s-vector is motivated by Slotine and Lie (1987) who introduced $\mathbf{s}$ as a measure of tracking when designing their adaptive robot controller. It turns out that this transformation has the nice property of transformation the nonlinear state-space model to the form:

$$
\begin{aligned}
\mathbf{M} \dot{\mathbf{s}}+\mathbf{D s} & =\mathbf{M} \dot{\boldsymbol{v}}+\mathbf{D}(\boldsymbol{v}) \boldsymbol{v}-\mathbf{M} \dot{\boldsymbol{v}}_{r}-\mathbf{D}(\boldsymbol{v}) \boldsymbol{v}_{r} \\
& =\mathbf{B u}-\mathbf{M} \dot{\boldsymbol{v}}_{r}-\mathbf{D}(\boldsymbol{v}) \boldsymbol{v}_{r}-\mathbf{K}(\boldsymbol{\eta}) \boldsymbol{\eta}
\end{aligned}
$$

where $\mathbf{v}_{r}$ can be interpreted as a "virtual" reference trajectory:

$$
\begin{aligned}
\boldsymbol{v}_{r} & =\boldsymbol{v}-\mathbf{s} \\
& =\boldsymbol{v}_{d}-\Lambda \tilde{\boldsymbol{\eta}}
\end{aligned}
$$

The position error dynamics of Step 1 can therefore be written:

$$
\begin{aligned}
\dot{\tilde{\boldsymbol{\eta}}} & =\boldsymbol{v}-\boldsymbol{v}_{d} \\
& =\mathbf{s}+\alpha_{1}-\boldsymbol{v}_{d} \quad\left(\alpha_{1}=v_{r}=\boldsymbol{v}-\mathbf{s}\right) \\
& =-\Lambda \tilde{\boldsymbol{\eta}}+\mathbf{s}
\end{aligned}
$$

Hence

$$
V_{1}=\frac{1}{2} \tilde{\boldsymbol{\eta}}^{T} \mathbf{K}_{p} \tilde{\boldsymbol{\eta}}, \mathbf{K}_{p}=\mathbf{K}_{p}^{T}>\mathbf{0}
$$

and

$$
\begin{aligned}
\dot{V}_{1} & =\tilde{\boldsymbol{\eta}}^{T} \mathbf{K}_{p} \dot{\tilde{\boldsymbol{\eta}}} \\
& =\tilde{\boldsymbol{\eta}}^{T} \mathbf{K}_{p}(-\Lambda \tilde{\boldsymbol{\eta}}+\mathbf{s}) \\
& =-\tilde{\boldsymbol{\eta}}^{T} \mathbf{K}_{p} \Lambda \tilde{\boldsymbol{\eta}}+\mathbf{s}^{T} \mathbf{K}_{p} \tilde{\boldsymbol{\eta}}
\end{aligned}
$$

Step 2:

In the second step we choose a Lyapunov function candidate motivated by the "pseudo" kinetic energy, that is:

$$
\begin{gathered}
V_{2}=V_{1}+\frac{1}{2} \mathbf{s}^{T} \mathbf{M s}, \mathbf{M}=\mathbf{M}^{T}>0 \\
\dot{V}_{2}=\dot{V}_{1}+\mathbf{s}^{T} \mathbf{M} \dot{\mathbf{s}} \\
=-\mathbf{s}^{T}\left(\mathbf{B u}-\mathbf{M} \dot{\boldsymbol{v}}_{r}-\mathbf{D}(\boldsymbol{v}) \boldsymbol{v}_{r}-\mathbf{K}(\boldsymbol{\eta}) \boldsymbol{\eta}-\mathbf{D}(\boldsymbol{v}) \mathbf{s}\right)-\tilde{\boldsymbol{\eta}}^{T} \mathbf{K}_{p} \Lambda \tilde{\boldsymbol{\eta}}+\mathbf{s}^{T} \mathbf{K}_{r} \tilde{\boldsymbol{\eta}} \\
=-\mathbf{s}^{T} \mathbf{D}(\boldsymbol{v}) \mathbf{s}+\mathbf{s}^{T}\left(\mathbf{B u}-\mathbf{M} \dot{\boldsymbol{v}}_{r}-\mathbf{D}(\boldsymbol{v}) \boldsymbol{v}_{r}-\mathbf{K}(\boldsymbol{\eta}) \boldsymbol{\eta}+\mathbf{K}_{p} \tilde{\boldsymbol{\eta}}\right)-\tilde{\boldsymbol{\eta}}^{T} \mathbf{K}_{p} \Lambda \tilde{\boldsymbol{\eta}}
\end{gathered}
$$

Hence, we are ready to propose a control law e.g.:

$$
\mathbf{B u}=\mathbf{M} \dot{\boldsymbol{v}}_{r}+\mathbf{D}(\boldsymbol{v}) \boldsymbol{v}_{r}+\mathbf{K}(\boldsymbol{\eta}) \boldsymbol{\eta}_{d}-\mathbf{K}_{p} \tilde{\boldsymbol{\eta}}-\mathbf{K}_{d} \mathbf{S}
$$

where $\mathbf{K}_{d}>0$. This results in:

$$
\begin{aligned}
\dot{V}_{2} & =-\mathbf{s}^{T}\left(\mathbf{D}(\boldsymbol{v})+\mathbf{K}_{d}\right) \mathbf{s}-\tilde{\boldsymbol{\eta}}^{T}\left(\mathbf{K}(\boldsymbol{\eta})+\mathbf{K}_{p} \Lambda\right) \tilde{\boldsymbol{\eta}} \\
& <0, \forall \mathbf{s} \neq \mathbf{0}, \tilde{\boldsymbol{\eta}} \neq \mathbf{0}
\end{aligned}
$$

Since $V_{2}$ is positive definite and $\dot{V}_{2}$ is negative definite it follows from Theorem 5 that the cquilibrium point $(\tilde{\boldsymbol{\eta}}, \mathbf{s})=(\mathbf{0}, \mathbf{0})$ is GES. Morcover, convergence of $\mathbf{s} \rightarrow \mathbf{0}$ and 
$\tilde{\eta} \rightarrow \mathbf{0}$ implies that $\tilde{\mathbf{v}} \rightarrow \mathbf{0}$. When implementing the control law (141) it is assumed that B has an inverse $(r \geq n)$ :

$$
\mathbf{B}^{\dagger}=\mathbf{B}^{T}\left(\mathbf{B} \mathbf{B}^{T}\right)^{-1}
$$

or that $\mathbf{B}^{-1}$ exists for the square case $r=n$. Hence;

$$
\mathbf{u}=\mathbf{B}^{\dagger}\left[\mathbf{M} \dot{\boldsymbol{v}}_{r}+\mathbf{D}(\boldsymbol{v}) \boldsymbol{v}_{r}+\mathbf{K}(\boldsymbol{\eta}) \boldsymbol{\eta}_{d}-\mathbf{K}_{p} \tilde{\boldsymbol{\eta}}-\mathbf{K}_{d} \mathbf{s}\right]
$$

\section{Error dynamics}

The error dynamics $\mathbf{z}_{1}=\tilde{\boldsymbol{\eta}}$ and $\mathbf{z}_{2}=\mathbf{s}$ becomes non-autonomous:

$$
\begin{aligned}
{\left[\begin{array}{cc}
\mathbf{K}_{p} & \mathbf{0} \\
\mathbf{0} & \mathbf{M}
\end{array}\right]\left[\begin{array}{l}
\dot{\mathbf{z}}_{1} \\
\dot{\mathbf{z}}_{2}
\end{array}\right] } & =-\left[\begin{array}{cc}
\mathbf{K}(\boldsymbol{\eta})+\mathbf{K}_{p} \Lambda & \mathbf{0} \\
\mathbf{0} & \mathbf{D}(\boldsymbol{v})+\mathbf{K}_{d}
\end{array}\right]\left[\begin{array}{l}
\mathbf{z}_{1} \\
\mathbf{z}_{2}
\end{array}\right]+\left[\begin{array}{cc}
\mathbf{0} & \mathbf{K}_{p} \\
-\mathbf{K}_{p} & \mathbf{0}
\end{array}\right]\left[\begin{array}{l}
\mathbf{z}_{1} \\
\mathbf{z}_{2}
\end{array}\right] \\
\mathscr{M} \dot{\mathbf{z}} & =-\mathscr{K}(\boldsymbol{v}, \boldsymbol{\eta}) \mathbf{z}+\mathscr{S} \mathbf{z}
\end{aligned}
$$

The error dynamics is GES since:

$$
\begin{aligned}
V(\mathbf{z}) & =\frac{1}{2} \mathbf{z}^{T} \mathscr{M} \mathbf{z} \\
\dot{V}(\mathbf{z}) & =\mathbf{z}^{T}[-\mathscr{H}(\boldsymbol{v}, \boldsymbol{\eta}) \mathbf{z}+\mathscr{S} \mathbf{z}] \\
& =-\mathbf{z}^{T} \mathscr{K}(\boldsymbol{v}, \boldsymbol{\eta}) \mathbf{z} \\
& <0, \forall \mathbf{z} \neq \mathbf{0}
\end{aligned}
$$

where we have used the skew-symmetric property $\mathbf{z}^{T} \mathscr{S} \mathbf{z}=0, \forall \mathbf{z}$.

\subsubsection{Dynamic positioning of ships}

Conventional ship control systems are designed under the assumption that the kinematic and dynamic equations of motion can be linearized such that gain-scheduling techniques and optimal control theory can be applied. This is not a good assumption for tracking applications where the surge and sway positions $(x, y)$ and yaw angle $\Psi$ must be controlled simultaneously, see Fossen and Grøvlen (1997). The main reason for this, is that the rotation matrix in yaw $\mathbf{J}(\boldsymbol{\eta})$, typically must be linearized about 36 operating points (steps of 10 degrees) to cover the whole circle arc with adequate accuracy. In addition to this, assumptions like linear damping and negligible Coriolis and centripetal forces are only good for low-speed applications, that is station-keeping and dynamic positioning (DP). These limitations clearly motivate a nonlinear design. MIMO nonlinear backstepping designs can be used for this purpose by exploiting nonlinear system properties like symmetry of the inertia matrix, dissipative damping and skew-symmetry of the Coriolis and centripetal matrix, see Fossen (1994), and Fossen and Fjellstad (1995).

\section{Vectorial backstepping of ships in 3 DOF}

Consider a surface ship described by the following model class, Fossen (1994):

$$
\begin{aligned}
\dot{\boldsymbol{\eta}} & =\mathbf{J}(\boldsymbol{\eta}) \boldsymbol{v} \\
\mathbf{M} \dot{\boldsymbol{v}}+\mathbf{C}(\boldsymbol{v}) \boldsymbol{v}+\mathbf{D}(\boldsymbol{v}) \boldsymbol{\eta} & =\mathbf{B u}
\end{aligned}
$$

This model describes the motion of a surface ship in 3 degrees of freedom (DOF) 
where $v=[u, v, r]^{T}$ is the velocity vector decomposed in the body-fixed reference frame, $\boldsymbol{\eta}=[x, y, \Psi]^{T}$ is the position/attitude vector decomposed in Earth-fixed coordinates and $\mathbf{u} \in \mathfrak{R}^{r}$ is a vector of control inputs (azimuth thrusters, main propellers and tunnel thrusters). It is assumed that $\mathbf{B} \in \mathfrak{R}^{3 \times r}$ and $r \geq 3$ such that the ship is fully actuated or overactuated. The model matrices are defined according to (Fossen, 1994):

$$
\begin{aligned}
\mathbf{M} & =\left[\begin{array}{ccc}
m-X_{\dot{u}} & 0 & 0 \\
0 & m-Y_{\dot{v}} & m x_{G}-Y_{\dot{r}} \\
0 & m x_{G}-Y_{\dot{r}} & I_{\mathbf{z}}-N_{\dot{r}}
\end{array}\right] \\
\mathbf{C}(v) & =\left[\begin{array}{ccc}
0 & 0 & -\left(m-Y_{\dot{v}}\right) v-\left(m x_{G}-Y_{\dot{r}}\right) r \\
0 & 0 & \left(m-X_{\dot{u}}\right) u \\
\left(m-Y_{\dot{v}}\right) v+\left(m x_{G}-Y_{\dot{r}}\right) r-\left(m-X_{u}\right) u & 0
\end{array}\right] \\
\mathbf{D}(v) & =\left[\begin{array}{ccc}
-X_{u} & 0 & 0 \\
0 & -Y_{v} & -Y_{r} \\
0 & -Y_{r} & -N_{r}
\end{array}\right]+\mathbf{D}_{n}(v) \\
\mathbf{J}(\eta) & =\left[\begin{array}{ccc}
\cos \Psi & -\sin \Psi & 0 \\
\sin \Psi & \cos \Psi & 0 \\
0 & 0 & 1
\end{array}\right]
\end{aligned}
$$

where $\mathbf{D}_{n}(v) \geq 0$ is a matrix of nonlinear damping terms. The nonlinear system (148)-(149) satisfy the following properties:

(i) $\mathbf{M}=\mathbf{M}^{T}$ is positive definite $\Rightarrow \mathbf{x}^{T} \mathbf{M x}>0, \forall \mathbf{x} \neq \mathbf{0}$

(ii) $\mathbf{C}(v)=-\mathbf{C}^{T}(v)$ is skew symmetrical $\Rightarrow \mathbf{x}^{T} \mathbf{C}(v) \mathbf{x}=0, \forall \mathbf{x}$

(iii) $\mathbf{D}(v)$ is strictly positive $\Rightarrow \mathbf{x}^{T} \mathbf{D}(v) \mathbf{x}=\frac{1}{2} \mathbf{x}^{T}\left[\mathbf{D}(v)+\mathbf{D}^{T}(v)\right] \mathbf{x}>0, \forall \mathbf{x} \neq \mathbf{0}$

(iv) $\mathbf{B B}^{T}$ is non-singular

(v) $\mathbf{J}(\boldsymbol{\eta})$ is the rotation matrix in yaw $\Rightarrow \mathbf{J}^{-1}(\boldsymbol{\eta})=\mathbf{J}^{T}(\boldsymbol{\eta})$

The control objective is to track a smooth bounded reference trajectory given by $\ddot{\boldsymbol{\eta}}_{d}, \dot{\boldsymbol{\eta}}_{d}$ and $\boldsymbol{\eta}_{d}$.

\section{New state variables}

The main difference between the ship tracking control problem and the nonlinear mass-damper-spring system is that the kinematic equation $\dot{\boldsymbol{\eta}}=\mathbf{J}(\boldsymbol{\eta}) \boldsymbol{v}$ is nonlinear for the ship. This problem can, however, be solved by applying a nonlinear state transformation motivated by the mass-damper-spring example in Section 2.2.2. Define two virtual reference trajectories as:

$$
\begin{aligned}
& \dot{\boldsymbol{\eta}}_{r} \triangleq \dot{\boldsymbol{\eta}}_{d}-\Lambda \tilde{\boldsymbol{\eta}} \\
& \boldsymbol{v}_{r} \triangleq \mathbf{J}^{-1}(\boldsymbol{\eta}) \dot{\boldsymbol{\eta}}_{r}
\end{aligned}
$$

where $\tilde{\boldsymbol{\eta}}=\boldsymbol{\eta}-\boldsymbol{\eta}_{d}$ is the Earth-fixed tracking error and $\Lambda>0$ is a diagonal design matrix. Furthermore, let $\mathbf{s}$ denote a measure of tracking defined according to:

$$
\mathbf{S} \triangleq \dot{\boldsymbol{\eta}}-\dot{\boldsymbol{\eta}}_{r}=\dot{\tilde{\boldsymbol{\eta}}}+\Lambda \tilde{\boldsymbol{\eta}}
$$

The marine vehicle dynamics (148)-(149) can then be written, Fossen (1994):

$$
\mathbf{M}_{\eta}(\boldsymbol{\eta}) \ddot{\boldsymbol{\eta}}+\mathbf{C}_{\eta}(\boldsymbol{v}, \boldsymbol{\eta}) \dot{\boldsymbol{\eta}}+\mathbf{D}_{\eta}(\boldsymbol{v}, \boldsymbol{\eta}) \dot{\boldsymbol{\eta}}=\mathbf{J}^{-T}(\boldsymbol{\eta}) \mathbf{B u}
$$




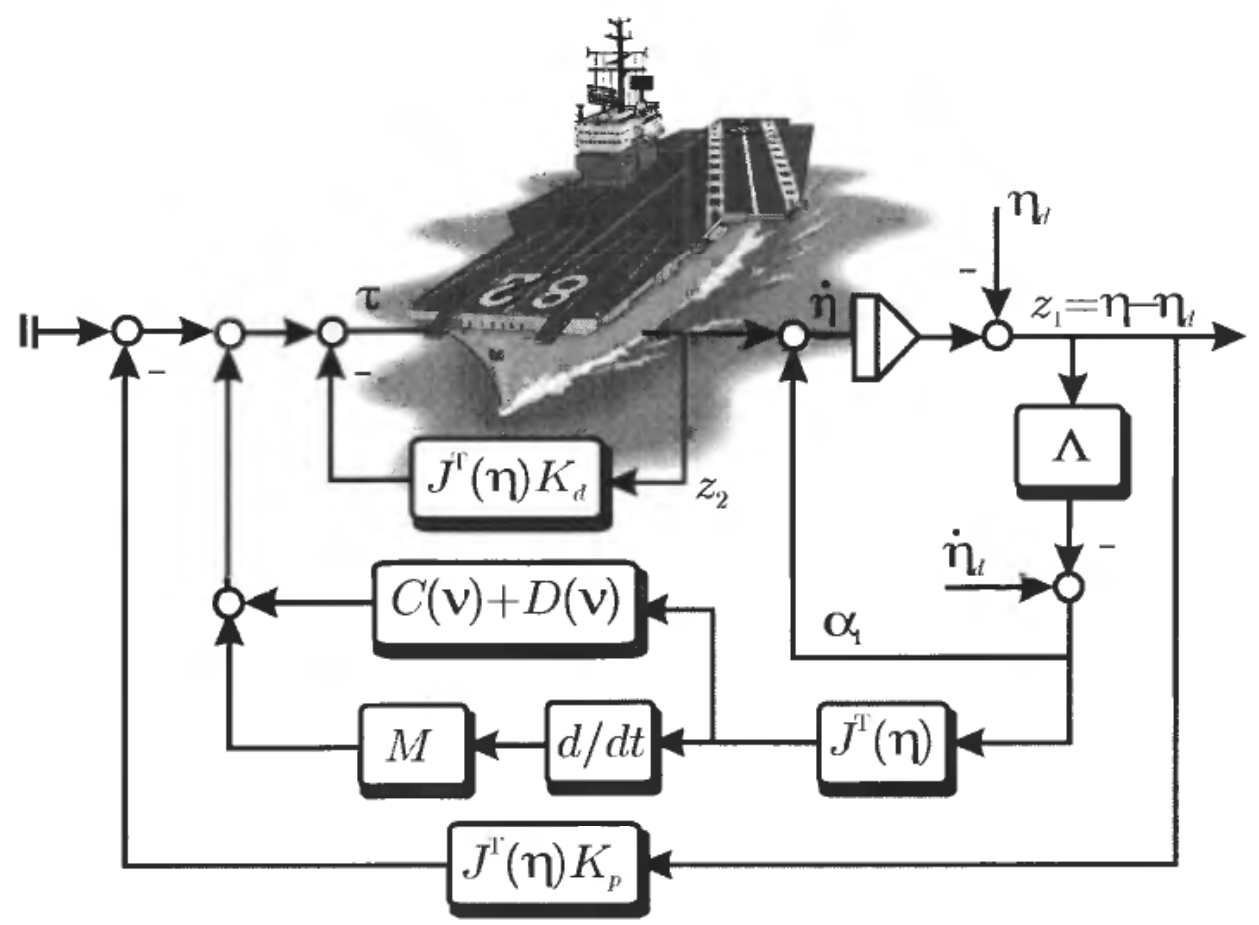

Figure 8. Nonlinear vectorial backstepping applied to dynamic positioning of ships.

where the Earth-fixed model matrices are defined as:

$$
\begin{aligned}
\mathbf{M}_{\eta}(\boldsymbol{\eta}) & =\mathbf{J}^{-T}(\boldsymbol{\eta}) \mathbf{M} \mathbf{J}^{-1}(\boldsymbol{\eta}) \\
\mathbf{C}_{\eta}(\boldsymbol{v}, \boldsymbol{\eta}) & =\mathbf{J}^{-\boldsymbol{T}}(\boldsymbol{\eta})\left[\mathbf{C}(\boldsymbol{v})-\mathbf{M} \mathbf{J}^{-1}(\boldsymbol{\eta}) \mathbf{J}(\boldsymbol{\eta})\right] \mathbf{J}^{-1}(\boldsymbol{\eta}) \\
\mathbf{D}_{\boldsymbol{\eta}}(\boldsymbol{v}, \boldsymbol{\eta}) & =\mathbf{J}^{-T}(\boldsymbol{\eta}) \mathbf{D}(\boldsymbol{v}) \mathbf{J}^{-1}(\boldsymbol{\eta})
\end{aligned}
$$

Hence, the marine vehicle dynamics takes the following form:

$$
\begin{aligned}
\mathbf{M}_{\eta}(\boldsymbol{\eta}) \dot{\mathbf{s}}= & -\mathbf{C}_{\eta}(\boldsymbol{v}, \boldsymbol{\eta}) \mathbf{s}-\mathbf{D}_{\eta}(\boldsymbol{v}, \boldsymbol{\eta}) \mathbf{s}+\mathbf{J}^{-T}(\boldsymbol{\eta}) \mathbf{B u} \\
& -\mathbf{M}_{\eta}(\boldsymbol{\eta}) \ddot{\boldsymbol{\eta}}_{r}-\mathbf{C}_{\eta}(\boldsymbol{v}, \boldsymbol{\eta}) \dot{\boldsymbol{\eta}}_{r}-\mathbf{D}_{\eta}(\boldsymbol{v}, \boldsymbol{\eta}) \dot{\boldsymbol{\eta}}_{r}
\end{aligned}
$$

or equivalently:

$$
\left.\mathbf{M}_{\eta}(\eta) \dot{\mathbf{s}}=-\mathbf{C}_{\eta}(\boldsymbol{v}, \boldsymbol{\eta}) \mathbf{s}-\mathbf{D}_{\eta}(\boldsymbol{v}, \boldsymbol{\eta}) \mathbf{s}+\mathbf{J}^{-T}(\boldsymbol{\eta})\left[\mathbf{B u}-\mathbf{M} \dot{v}_{r}-\mathbf{C} \boldsymbol{v}\right) \boldsymbol{v}_{r}-\mathbf{D}(v) v_{r}\right]
$$

\section{Step 1:}

Define the virtual control vector:

$$
\dot{\boldsymbol{\eta}}=\mathbf{J}(\boldsymbol{\eta}) \boldsymbol{v} \underline{\underline{\wedge}} \mathbf{s}+\alpha_{1}
$$

where $\alpha_{1}$ is a stabilizing vector field which can be chosen as:

$$
\alpha_{1}=\dot{\boldsymbol{\eta}}_{r}=\dot{\boldsymbol{\eta}}_{d}-\Lambda \tilde{\boldsymbol{\eta}}
$$

Hence (156) can be written:

$$
\dot{\tilde{\boldsymbol{\eta}}}=-\Lambda \tilde{\boldsymbol{\eta}}+\mathbf{s}
$$

Consider the Lyapunov function candidate:

$$
V_{1}=\frac{1}{2} \tilde{\boldsymbol{\eta}}^{T} \mathbf{K}_{p} \tilde{\boldsymbol{\eta}}
$$




$$
\dot{\boldsymbol{V}}=\dot{\boldsymbol{\eta}}^{T} \mathbf{K}_{p} \dot{\overline{\boldsymbol{\eta}}}=-\tilde{\boldsymbol{\eta}}^{T} \mathbf{K}_{p} \Lambda \tilde{\boldsymbol{\eta}}^{T} \mathbf{K}_{p} \mathbf{S}
$$

where $\mathbf{K}_{p}=\mathbf{K}_{p}^{T}>0$ is a design matrix.

\section{Step 2:}

Next consider the Lyapunov function candidate:

$$
\begin{gathered}
V_{2}=V_{1}+\frac{1}{2} \mathbf{s}^{T} \mathbf{M}_{\eta}(\boldsymbol{\eta}) \mathbf{s} \\
\dot{V}_{2}=-\tilde{\boldsymbol{\eta}}^{T} \mathbf{K}_{p} \Lambda \tilde{\boldsymbol{\eta}}+\mathbf{s}^{T}\left[\mathbf{K}_{p} \tilde{\boldsymbol{\eta}}+\mathbf{M}_{\eta}(\boldsymbol{\eta}) \dot{\mathbf{s}}+\frac{1}{2} \dot{\mathbf{M}}_{\eta}(\boldsymbol{\eta}) \mathbf{s}\right]
\end{gathered}
$$

Substitution of (155) into the expression from $\dot{V}_{2}$ yields:

$$
\begin{aligned}
\dot{V}_{2} & =-\tilde{\boldsymbol{\eta}}^{T} \mathbf{K}_{p} \Lambda \tilde{\boldsymbol{\eta}}+\mathbf{s}^{T}\left[\mathbf{K}_{p} \tilde{\boldsymbol{\eta}}+\frac{1}{2} \dot{\mathbf{M}}_{\eta} \mathbf{s}-\mathbf{C}_{\eta}(\boldsymbol{v}, \boldsymbol{\eta}) \mathbf{s}-\mathbf{D}_{\eta}(\boldsymbol{v}, \boldsymbol{\eta})\right] \mathbf{s} \\
& +\mathbf{s}^{T} \mathbf{J}^{-T}(\boldsymbol{\eta})\left[\mathbf{B u}-\mathbf{M} \dot{\boldsymbol{v}}_{r}-\mathbf{C}(\boldsymbol{v}) \boldsymbol{v}_{r}-\mathbf{D}(\boldsymbol{v}) \boldsymbol{v}_{r}\right]
\end{aligned}
$$

Using the fact that, Fossen (1994):

$$
\mathbf{s}^{T}\left(\frac{1}{2} \dot{\mathbf{M}}_{\eta}(\eta)-\mathbf{C}_{\eta}(\boldsymbol{v}, \boldsymbol{\eta})\right) \mathbf{s}=0, \quad \forall \mathbf{s}
$$

implies that (163) reduces to:

$$
\begin{aligned}
\dot{V}_{2} & =-\tilde{\boldsymbol{\eta}}^{T} \mathbf{K}_{p} \Lambda \tilde{\boldsymbol{\eta}}-\mathbf{s}^{T} \mathbf{D}_{\eta}(\boldsymbol{v}, \boldsymbol{\eta}) \mathbf{s} \\
& +\mathbf{s}^{T} \mathbf{J}^{-T}(\boldsymbol{\eta})\left[\mathbf{J}^{T}(\boldsymbol{\eta}) \mathbf{K}_{p} \tilde{\boldsymbol{\eta}}+\mathbf{B} \mathbf{u}-\mathbf{M} \dot{\boldsymbol{v}}_{r}-\mathbf{C}(\boldsymbol{v}) \boldsymbol{v}_{r}-\mathbf{D}(\boldsymbol{v}) \boldsymbol{v}_{r}\right]
\end{aligned}
$$

This suggests that the control law should be chosen as:

$$
\mathbf{u}=\mathbf{B}^{\dagger}\left[\mathbf{M} \dot{\boldsymbol{v}}_{r}+\mathbf{C}(\boldsymbol{v}) \boldsymbol{v}_{r}+\mathbf{D}(\boldsymbol{v}) \boldsymbol{v}_{r}-\mathbf{J}^{T}(\boldsymbol{\eta}) \mathbf{K}_{d} \mathbf{S}-\mathbf{J}^{T}(\boldsymbol{\eta}) \mathbf{K}_{r} \tilde{\boldsymbol{\eta}}\right]
$$

where $\mathbf{B}^{\dagger}$ is the pseudo-inverse:

$$
\mathbf{B}^{\dagger}=\mathbf{B}^{T}\left(\mathbf{B} \mathbf{B}^{T}\right)^{-1}
$$

Hence, the resulting expression for $\dot{V}_{2}$ takes the form:

$$
\begin{aligned}
\dot{V}_{2} & =-\tilde{\boldsymbol{\eta}}^{T} \mathbf{K}_{p} \Lambda \tilde{\boldsymbol{\eta}}-\mathbf{s}^{T}\left(\mathbf{D}_{\boldsymbol{\eta}}(\boldsymbol{v}, \boldsymbol{\eta})+\mathbf{K}_{d}\right) \mathbf{s} \\
& <0, \forall \tilde{\boldsymbol{\eta}} \neq \mathbf{0}, \mathbf{s} \neq \mathbf{0}
\end{aligned}
$$

\section{Error dynamics}

The error dynamics $\mathbf{z}_{1}=\tilde{\boldsymbol{\eta}}$ and $\mathbf{z}_{2}=\mathbf{s}$ becomes non-autonomous:

$$
\begin{aligned}
{\left[\begin{array}{cc}
\mathbf{K}_{p} & \mathbf{0} \\
\mathbf{0} & \mathbf{M}_{\eta}(\boldsymbol{\eta})
\end{array}\right]\left[\begin{array}{l}
\dot{\mathbf{z}}_{1} \\
\dot{\mathbf{z}}_{2}
\end{array}\right]=} & -\left[\begin{array}{cc}
\mathbf{K}_{p} \Lambda & \mathbf{0} \\
\mathbf{0} & \mathbf{C}_{\eta}(\boldsymbol{v}, \boldsymbol{\eta})+\mathbf{D}_{\eta}(\boldsymbol{v}, \boldsymbol{\eta})+\mathbf{K}_{d}
\end{array}\right]\left[\begin{array}{l}
\mathbf{z}_{1} \\
\mathbf{z}_{2}
\end{array}\right] \\
& +\left[\begin{array}{cc}
\mathbf{0} & \mathbf{K}_{p} \\
-\mathbf{K}_{p} & \mathbf{0}
\end{array}\right]\left[\begin{array}{l}
\mathbf{z}_{1} \\
\mathbf{z}_{2}
\end{array}\right] \\
\mathbb{I} & \\
\mathscr{M}(\boldsymbol{\eta}) \dot{\mathbf{z}}= & -\mathscr{K}(\boldsymbol{v}, \boldsymbol{\eta}) \mathbf{z}+\mathscr{S} \mathbf{z}
\end{aligned}
$$




\section{Set-point regulation (station-keeping)}

A special case of DP is station-keeping which is obtained by choosing:

$$
\boldsymbol{\eta}_{d}=\text { constant }
$$

For this case, the DP control law takes the following form:

$$
\begin{aligned}
\boldsymbol{\tau} & =\mathbf{M} \dot{\boldsymbol{v}}_{r}+\mathbf{C}(\boldsymbol{v}) \boldsymbol{v}_{r}+\mathbf{D}(\boldsymbol{v}) \boldsymbol{v}_{r}-\mathbf{J}^{T}(\boldsymbol{\eta}) \mathbf{K}_{d} \mathbf{S}-\mathbf{J}^{T}(\boldsymbol{\eta}) \mathbf{K}_{p} \tilde{\boldsymbol{\eta}} \\
\mathbf{u} & =\mathbf{B}^{\dagger} \boldsymbol{\tau}
\end{aligned}
$$

where

$$
\begin{aligned}
& \mathbf{s}=\dot{\boldsymbol{\eta}}-\Lambda \tilde{\boldsymbol{\eta}}=\mathbf{J}(\boldsymbol{\eta}) \boldsymbol{v}-\Lambda \tilde{\boldsymbol{\eta}} \\
& \boldsymbol{v}_{r}=\mathbf{J}^{T}(\boldsymbol{\eta}) \dot{\boldsymbol{\eta}}_{r}=\mathbf{J}^{T}(\boldsymbol{\eta}) \alpha_{1} \\
& \boldsymbol{\alpha}_{1}=-\Lambda \tilde{\boldsymbol{\eta}}
\end{aligned}
$$

\section{Locally optimal backstepping design}

Optimal and robust control of linear systems is well established. For nonlinear systems, however, optimal control design is a relatively new field of research. The main obstacle in achieving optimal control of nonlinear systems is obtaining a solution to the Hamilton-Jacobi-Bellman (HJB) equation, and in the case of robust optimal control, obtaining a solution of the Hamilton-Jacobi-Isaacs (HJI) equation. This can be circumvented by an inverse optimal approach.

In contrast to linear methods, where the cost functional to be minimized is specified in advance, the inverse approach is to first find a stabilizing control law and then determine the cost functional which is minimized by that particular control law. Using Lyapunov methods, these inverse designs construct the value function which solves the corresponding HJB or HJI equations. In Ezal, Pan and Kokotović (1997) a locally optimal and globally inverse optimal design methodology were derived for SISO nonlinear systems in strict-feedback form. This is a constructive design procedure which permits the control designer to specify the local behavior of the system. In this design, the cost functional for the linearized system is specified, and at the same time a solution for global stability and optimality of the nonlinear system is sought. Thus, this new method is an extension of well-known linear quadratic (LQ) and $\mathscr{H}_{\infty}$-methods to nonlinear systems in strict feedback form.

The new design methodology is called locally optimal backstepping (LOB) and it includes two design mechanisms. First, the system is transformed into new coordinates by recursive backstepping. Here, each stabilizing function consists of one linear term and one nonlinear term. The linear term is pre-defined, since the behavior close to the working point is given by the linear cost functional. Simultaneously, the nonlinear term -of the stabilizing functions are used in an inverse optimal manner.

The LOB design for SISO systems presented below is based on the Ph.D. dissertation of Ezal (1998). In this work, it is demonstrated that the LOB design can be extended to tracking and output feedback control. In Strand, Ezal, Fossen and Kokotović (1998) the LOB procedure was extended to MIMO systems in block-strictfeedback form by applying the LOB design methodology to nonlinear optimal MIMO control of moored and free-floating ships. 


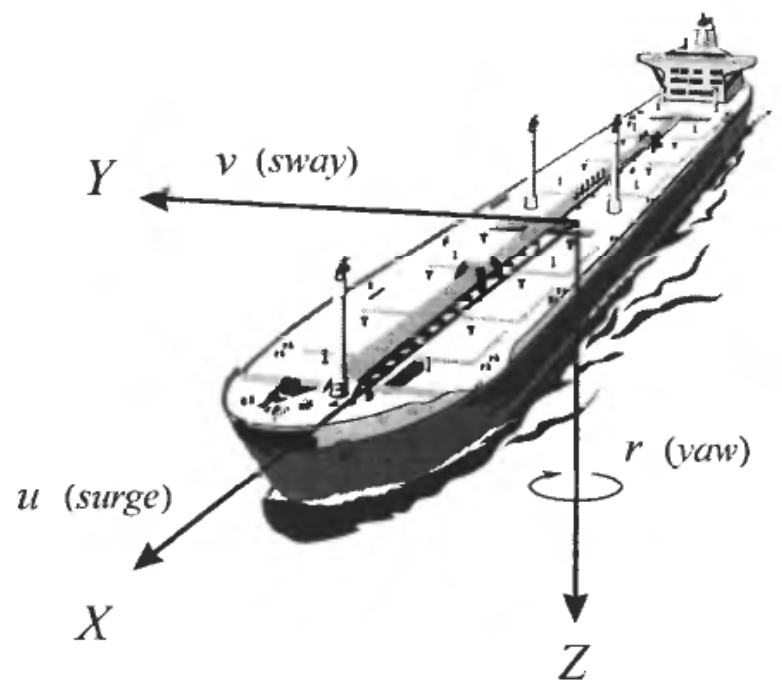

Figure 9. Free floating tanker.

\subsection{SISO optimal backstepping}

\subsubsection{Notation}

For SISO systems the following notation is adopted:

The $i \times i$ upper left sub-matrix of a matrix $\mathbf{A} \in \mathfrak{R}^{n \times n}$ is defined for all $i=1 \ldots n$ as:

$$
\mathbf{A}_{[i]}=\left[\begin{array}{ccc}
a_{11} & \ldots & a_{1 i} \\
\vdots & \ddots & \vdots \\
a_{i 1} & \ldots & a_{i i}
\end{array}\right]
$$

and the same notation is used for subsets of a state vector and a vector field, i.e. a state vector containing the first $i$-th element of $\mathbf{x} \in \mathfrak{R}^{n}$ as:

$$
\mathbf{x}_{[i]}=\left[x_{1}, \ldots, x_{i}\right]^{T}
$$

With a slight abuse of notation, the $i$-th row vector of $\mathbf{A}_{[i]}$ will be denoted as:

$$
\mathbf{a}_{i j]}=\left[a_{i 1}, \ldots, a_{i i}\right]
$$

The zero matrix of dimension $i \times i$ is denoted $\mathbf{O}_{[i]}$ for all $i=1 \ldots . . n$ and $\mathbf{O} \in \mathfrak{R}^{n \times n}$.

\subsubsection{Model Class}

In this section we consider SISO strict-feedback systems in the following form:

$$
\begin{aligned}
\dot{x}_{1}= & f_{1}\left(x_{1}\right)+x_{2} \\
\dot{x}_{2}= & f_{2}\left(x_{1}, x_{2}\right)+x_{3} \\
\dot{x}_{3}= & f_{3}\left(x_{1}, x_{2}, x_{3}\right)+x_{4} \\
& \vdots \\
\dot{x}_{n-1}= & f_{n-1}\left(x_{1}, x_{2}, \ldots, x_{n-1}\right)+x_{n} \\
\dot{x}_{n}= & f_{n}\left(x_{1}, x_{2}, \ldots, x_{k}\right)+b_{n} u
\end{aligned}
$$


This can be written compactly as:

$$
\dot{\mathbf{x}}=\mathbf{f}(\mathbf{x})+\mathbf{b} u
$$

Here $\mathbf{x}=\left[x_{1}, \ldots, x_{n}\right]^{T} \in \mathfrak{R}^{n}, u \in \mathfrak{R}$. It is assumed that $f_{i}$ are sufficiently smooth and that $f_{i}(0, \ldots, 0)=0$. Notice that this definition of strict-feedback systems deviates from Definition 2 in Section 2.1. The difference is that here all terms $g_{i}\left(x_{1}, \ldots, x_{i}\right)=1$ $(i=1 \ldots . . n-1)$ and $g_{n}\left(x_{1}, x_{2}, \ldots, x_{n}\right)=b_{n}$.

The system (182) can be re-written as:

$$
\dot{\mathbf{x}}=\mathbf{A x}+\mathbf{b} u+\tilde{\mathbf{f}}(\mathbf{x})
$$

where

$$
\begin{aligned}
& \mathbf{A}=\left.\frac{\partial \mathbf{f}}{\partial \mathbf{x}}\right|_{\mathbf{x}=\mathbf{0}}=\left[\begin{array}{cccccc}
a_{11} & 1 & 0 & \ldots & 0 & 0 \\
a_{21} & a_{22} & 1 & \ldots & 0 & 0 \\
a_{31} & a_{32} & a_{33} & \ldots & 0 & 0 \\
\vdots & \vdots & \vdots & \ddots & \vdots & \vdots \\
a_{n-1,1} & a_{n-1,2} & a_{n-1,3} & \ldots & a_{n-1, n-1} & 1 \\
a_{n, 1} & a_{n, 2} & a_{n, 3} & \ldots & a_{n, n-1} & a_{n, n}
\end{array}\right] \\
& \mathbf{b}=\left[0,0, \ldots, b_{n}\right]^{T}
\end{aligned}
$$

and $\tilde{\mathbf{f}}(\mathbf{x})=\mathbf{f}(\mathbf{x})-\mathbf{A x}$.

\subsubsection{Control Objectives}

O1: Global Objective. The ultimate control objective is to design a globally asymptotically stabilizing feedback control law $u=\mu(\mathbf{x})$ which minimizes the cost functional:

$$
J=\int_{0}^{\infty}\left[q(\mathbf{x})+r(\mathbf{x}) u^{2}\right] d t
$$

subject to the system dynamics (182). Here $q(\mathbf{x})$ and $r(\mathbf{x})$ are not specified beforehand, rather constructed during the control design. Thus this is an inverse approach that obtains an inverse optimal control law. The requirements are:

$$
q(\mathbf{x}) \text { is positive definite and } r(\mathbf{x})>0 \forall \mathbf{x}
$$

O2: Local Objective. The second control objective is to find a control law that minimizes the cost functional

$$
J_{l}=\int_{0}^{\infty}\left[\mathbf{x}^{T} \mathbf{Q} \mathbf{x}+R u_{I}^{2}\right] d t
$$

locally, i.e. near the origin $\mathbf{x}=0$, subject to the linearized system dynamics:

$$
\dot{\mathbf{x}}=\mathbf{A x}+\mathbf{b} u_{l}
$$

IIcre, local behavior of the closed-loop system is designed by specifying the state 


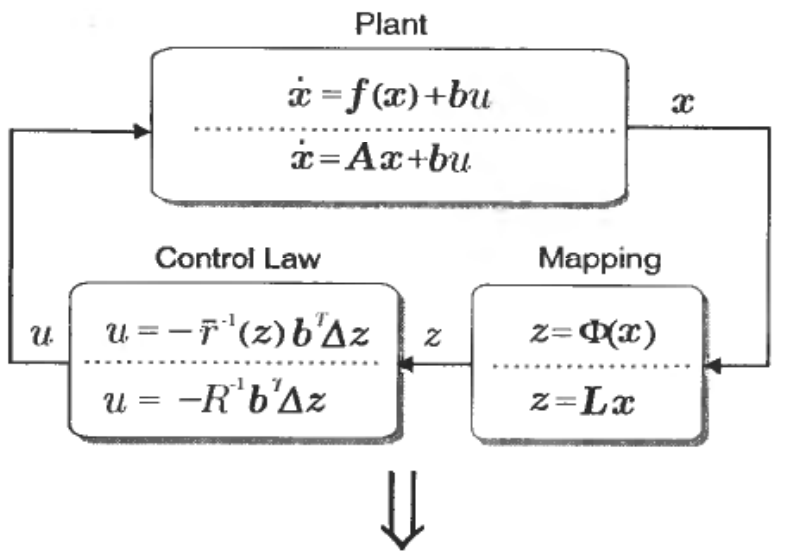

Cost functional

$$
\begin{aligned}
& J=\int_{0}^{x}\left[q(\boldsymbol{x})+r(\boldsymbol{x}) u^{2}\right] d t \\
& J=\int_{0}^{\alpha}\left[\boldsymbol{x}^{T} \boldsymbol{Q} \boldsymbol{x}+R \boldsymbol{u}^{2}\right] d t
\end{aligned}
$$

Figure 10. Relationship between linear LQ control (lower blocks) and nonlinear optimal backstepping (upper blocks).

cost matrix $\mathbf{Q}=\mathbf{Q}^{T}>0$ and the cost $R>0$ on control effort. Although not specified beforehand, $q(\mathbf{x})$ and $r(\mathbf{x})$ in $\mathrm{Ol}$ must satisfy the local objective by requiring that

$$
q_{x x}(0)=\left.\frac{1}{2} \frac{\partial^{2} q(x)}{\partial \mathbf{x}^{2}}\right|_{\mathrm{x}=0} ^{r(0)=R}=\mathbf{Q}
$$

\subsubsection{Locally Optimal Backstepping (LOB)}

A linear control law $u_{l}$ that meets the local control objective $\mathrm{O} 2$ can easily be found by solving the algebraic Riccati equation (ARE):

$$
\mathbf{P A}+\mathrm{A}^{T} \mathbf{P}-\mathbf{P b} R^{-1} \mathbf{b}^{T} \mathbf{P}+\mathbf{Q}=\mathbf{0}
$$

with $\mathbf{P}=\mathbf{P}^{T}>\mathbf{0}$. This solution exists provided that $(\mathbf{A}, \mathbf{b})$ is controllable and $(\mathbf{A}, \mathbf{Q})$ is observable. This linear quadratic (LQ) controller is:

$$
u_{l}=-R^{-1} \mathbf{b}^{T} \mathbf{P x}
$$

The global objective $\mathrm{O} 1 \mathrm{can}$ be met for systems in SISO strict-feedback form (177)-(181) by the LOB procedure. A mapping:

$$
\mathbf{z}=\Phi(\mathbf{x})
$$

into a new set of variables $\mathbf{z}$ is constructed by backstepping. It will be shown that this diffeomorphism can be written as:

$$
\mathbf{z}=\mathbf{L} \mathbf{x}+\tilde{\Phi}(\mathbf{x})
$$


where $\tilde{\Phi}(\mathbf{x})$ contains higher order terms. The constant matrix $\mathbf{L}$ is found by factorization of $\mathbf{P}$ into':

$$
\mathbf{P}=\mathbf{L}^{T} \mathbf{\Lambda} \mathbf{L}
$$

where

$$
\begin{aligned}
\boldsymbol{\Lambda} & =\left[\begin{array}{cccc}
\delta_{1} & 0 & \ldots & 0 \\
0 & \delta_{2} & \ddots & 0 \\
\vdots & \ddots & \ddots & \vdots \\
0 & \ldots & 0 & \delta_{n}
\end{array}\right]=\operatorname{diag}\left\{\delta_{1}, \ldots, \delta_{n}\right\} \\
\mathbf{L} & =\left[\begin{array}{cccc}
1 & 0 & \ldots & 0 \\
-l_{11} & 1 & \ddots & 0 \\
\vdots & \ddots & \ddots & \vdots \\
-l_{n-1,1} & \ldots & -l_{n-1, n-1} & 1
\end{array}\right]
\end{aligned}
$$

Applying the transformation $\mathbf{z}=\mathbf{L x}$ to the linear system (189) yields:

$$
\dot{\mathbf{z}}=\overline{\mathbf{A}} \mathbf{z}+\mathbf{b} u
$$

where $\overline{\mathbf{A}}=\mathbf{L A} \mathbf{L}^{-1}$. The structure of $\overline{\mathbf{A}}$ is

$$
\overline{\mathbf{A}}=\left[\begin{array}{ccccc}
\bar{a}_{11} & 1 & 0 & \ldots & 0 \\
\bar{a}_{21} & \bar{a}_{22} & 1 & \ddots & \vdots \\
\vdots & \ddots & \ddots & \ddots & 0 \\
\vdots & \ddots & \ddots & \bar{a}_{n-1, n-1} & 1 \\
\bar{a}_{n, 1} & \cdots & \ldots & \bar{a}_{n, n-1} & \bar{a}_{n n}
\end{array}\right]
$$

By replacing $\mathbf{P}$ with $\mathbf{L}^{T} \Delta \mathbf{L}$, and pre-multiplying with $\mathbf{L}^{-T}$ and post-multiplying with $\mathbf{L}^{-1}$, the ARE (190) transforms to:

$$
\overline{\mathbf{A}}^{T} \boldsymbol{\Delta}+\boldsymbol{\Delta} \overline{\mathbf{A}}-\mathbf{\Delta} \mathbf{b} R^{-1} \mathbf{b}^{T} \boldsymbol{\Delta}+\overline{\mathbf{Q}}=\mathbf{0}
$$

where $\overline{\mathbf{Q}}=\mathbf{L}^{-T} \mathbf{Q} \mathbf{L}^{-1}=\overline{\mathbf{Q}}^{T}>\mathbf{0}$.

The linear part $\mathbf{L x}$ of the diffeomorphism (193) is given by the factorization of $\mathbf{P}$. For the nonlinear system (186), however, the transformation into new variables must be done by a recursive backstepping procedure, which is the topic of the next section. It will be shown that the nonlinear system (186) can be represented as:

$$
\dot{\mathbf{z}}=\overline{\mathbf{A}} \mathbf{z}+\mathbf{b} u+\mathbf{f}(\mathbf{z})
$$

where

$$
\breve{f}(\mathbf{z})=\left[0,0, \ldots, \check{f}_{n}(\mathbf{z})\right]^{T}
$$

In recursive backstepping each stabilizing function $\alpha_{i}$ must be chosen to match the linear mapping, whereas the nonlinear terms can be chosen freely. For simplicity we choose to cancel the nonlinearities at each step $i=1 \ldots n-1$. It is, however, possible to keep "good damping" terms at each step of the backstepping.

\footnotetext{
${ }^{1}$ It can be shown that $\Delta^{1 / 2} \mathbf{L}=\mathbf{U}^{-T}$, where $\mathbf{U}$ is a unique Cholesky factor of $\mathbf{P}^{-1}=\mathbf{U}^{T} \mathbf{U}$. Therefore the pair $(\Lambda, \mathbf{L})$ is unique. Matlab command: $U=\operatorname{chol}(\operatorname{inv}(P))$.
} 


\section{Lyapunov design}

Let:

$$
\bar{V}=\mathbf{z}^{T} \Delta \mathbf{z}
$$

be a Lyapunov function for the transformed system (200). Thus:

$$
\dot{\bar{V}}=\mathbf{z}^{T}\left(\overline{\mathbf{A}}^{T} \Delta+\Delta \overline{\mathbf{A}}\right) \mathbf{z}+2 u \mathbf{b}^{T} \Delta \mathbf{z}+2 \check{\mathbf{f}}^{T}(\mathbf{z}) \Delta \mathbf{z}
$$

By substitution of the ARE (199) we get:

$$
\dot{\bar{V}}=-\mathbf{z}^{T} \overline{\mathbf{Q}} \mathbf{z}+\mathbf{z}^{T} \Delta \mathbf{b} R^{-1} \mathbf{b}^{T} \Delta \mathbf{z}+2 u \mathbf{b}^{T} \Delta \mathbf{z}+2 \check{\mathbf{f}}^{T}(\mathbf{z}) \Delta \mathbf{z}
$$

Due to the structure of $\check{\mathbf{f}}(\mathbf{z})$ and $\mathbf{b}$ we get:

$$
\begin{aligned}
\mathbf{z}^{T} \Delta \mathbf{b} R^{-1} \mathbf{b}^{T} \Delta \mathbf{z} & =R^{-1} b_{n}^{2} \delta_{n}^{2} z_{n}^{2} \\
2 u \mathbf{b}^{T} \Delta \mathbf{z} & =2 b_{n} \delta_{n} u z_{n} \\
2 \breve{\mathbf{f}}^{T}(\mathbf{z}) \Delta \mathbf{z} & =2 \check{f}_{n}(\mathbf{z}) \delta_{n} z_{n}
\end{aligned}
$$

such that:

$$
\dot{\bar{V}}=-\mathbf{z}^{T} \overline{\mathbf{Q}} \mathbf{z}+R^{-1} b_{n}^{2} \delta_{n}^{2} z_{n}^{2}+2 b_{n} \delta_{n} u z_{n}+2 \check{f}_{n}(\mathbf{z}) \delta_{n} z_{n}
$$

By adding and subtracting $\bar{r}(\mathbf{z}) u^{2}$ and completion of the squares such that

$$
\bar{r}(\mathbf{z}) u^{2}+2 b_{n} \delta_{n} u z_{n}=\bar{r}(\mathbf{z})\left(u+r^{-1}(\mathbf{z}) b_{n} \delta_{n} z_{n}\right)^{2}+r^{-1}(\mathbf{z}) b_{n}^{2} \delta_{n}^{2} z_{n}^{2}
$$

the expression for $\dot{\bar{V}}$ becomes:

$$
\begin{aligned}
\dot{\bar{V}}= & -\mathbf{z}^{T} \overline{\mathbf{Q}} \mathbf{z}+\bar{r}(\mathbf{z})\left(u+r^{-1}(\mathbf{z}) b_{n} \delta_{n} z_{n}\right)^{2} \\
& -\left(r^{-1}(\mathbf{z})-R^{-1}\right) b_{n}^{2} \delta_{n}^{2} z_{n}^{2}+2 f_{n}(\mathbf{z}) \delta_{n} z_{n}-\bar{r}(\mathbf{z}) u^{2}
\end{aligned}
$$

The second term in (206) is rendered equal to zero by choosing the optimal control law as:

$$
u=-\overline{\boldsymbol{r}}^{-1}(\mathbf{z}) \mathbf{b}^{T} \Delta \mathbf{z}=-\boldsymbol{r}^{-1}(\mathbf{z}) b_{n} \delta_{n} z_{n}
$$

Hence:

$$
\dot{\bar{V}}=\underbrace{-\mathbf{z}^{T} \overline{\mathbf{Q}} \mathbf{z}-\left(\overline{\boldsymbol{r}}^{-1}(\mathbf{z})-R^{-1}\right) b_{n}^{2} \delta_{n}^{2} z_{n}^{2}+2 \check{f}_{n}(\mathbf{z}) \delta_{n} z_{n}}_{-\dot{q}(\mathbf{z})}-\bar{r}(\mathbf{z}) u^{2}
$$

It remains to show that $\bar{q}(\mathbf{z})$ is a positive definite function and $\bar{r}(\mathbf{z})$ is a strictly positive function. This is necessary for obtaining local optimality and global inverse optimality.

\section{Cost functional weights}

Based on (208) it remains to determine $\bar{q}(\mathbf{z})$ and $\bar{r}(\mathbf{z})$ such that

$$
\bar{q}(\mathbf{z}) \text { is positive definite, and } \bar{r}(\mathbf{z})>0 \quad \forall \mathbf{z}
$$

which locally satisfies

$$
\begin{aligned}
& \bar{r}(\mathbf{0})=R \\
& \bar{q}_{z z}(\mathbf{0})=\left.\frac{1}{2} \frac{\partial \bar{q}(\mathbf{z})}{\partial \mathbf{z}^{2}}\right|_{\mathrm{z}=\mathbf{0}}=\overline{\mathbf{Q}}
\end{aligned}
$$


The solution is completion of the squares and "stealing" positive definiteness from $\overline{\mathbf{Q}}$. It will be shown that $\bar{q}(\mathbf{z})$ can be written as:

$$
\bar{q}(\mathbf{z})=\bar{q}_{0}(\mathbf{z})+\left(r^{-1}(\mathbf{z})-R^{-1}-\bar{\sigma}(\mathbf{z})\right) b_{n}^{2} \delta_{n}^{2} z_{n}^{2}
$$

where $\bar{\sigma}(\mathbf{0})=0$ and $\bar{q}_{0}(\mathbf{z})$ is positive definite. If $\bar{r}(\mathbf{z})$ is chosen such that:

$$
\begin{aligned}
\bar{r}^{-1}(\mathbf{z}) & \geq R^{-1}+\bar{\sigma}(\mathrm{z}), \\
\vec{r}(0) & =R, \\
\bar{r}(\mathbf{z}) & >0 \quad \forall \mathbf{z},
\end{aligned}
$$

then $\bar{q}(\mathbf{z})$ is positive definite and the origin $\mathbf{z}=\mathbf{0}$ of the nonlinear system (200) is rendered globally asymptotically stable (GAS).

\section{Proof}

Let $\mathbf{z}^{T} \overline{\mathbf{Q}} \mathbf{z}$ be written:

$$
\begin{aligned}
\mathbf{z}^{T} \overline{\mathbf{Q}} \mathbf{z} & =\left[\begin{array}{ll}
\mathbf{z}_{[n-1]}^{T} & \left.z_{n}\right]
\end{array}\right]\left[\begin{array}{cc}
\overline{\mathbf{Q}}_{[n-1]} & \overline{\mathbf{q}}_{1} \\
\overline{\mathbf{q}}_{1}^{T} & \bar{q}_{2}
\end{array}\right]\left[\begin{array}{c}
\mathbf{z}_{[n-1]} \\
z_{n}
\end{array}\right] \\
& \left.=\mathbf{z}_{[n-1}^{T}\right] \overline{\mathbf{Q}}_{[n-1]} \mathbf{z}_{[n-1]}+2 z_{n} \overline{\mathbf{q}}_{1}^{T} \mathbf{z}_{[n-1]}+\bar{q}_{2} z_{n}^{2}
\end{aligned}
$$

where $\mathbf{z}_{[i]}$ denotes a vector of the $i$ first vector elements of $\mathbf{z}$ and $\overline{\mathbf{Q}}_{[i]}$ denotes the $\boldsymbol{i}$-th upper sub-matrix of $\overline{\mathbf{Q}}$. The expression $\check{f}_{n}(\mathbf{z})$ can be factorized to:

$$
\check{f}_{n}(\mathbf{z})=\overline{\boldsymbol{\eta}}_{1}(\mathbf{z}) \mathbf{z}_{[n-1]}+\bar{\eta}_{2}(\mathbf{z}) z_{n}
$$

By completion of the squares with respect to $\mathbf{z}_{[n-1]}$ such that $\dot{\bar{V}}$ no longer contains cross-terms of $\mathbf{z}_{[n-1]}$ and $z_{n}$ we get:

$$
\begin{aligned}
-\mathbf{z}^{T} & \overline{\mathbf{Q}}_{\mathbf{z}}+2 \check{f}_{n}(\mathbf{z}) \delta_{n} z_{n} \\
= & -\mathbf{z}_{[n-1]}^{T} \overline{\mathbf{Q}}_{[n-1]} \mathbf{z}_{[n-1]}-2 z_{n} \overline{\mathbf{q}}_{1}^{T} \mathbf{z}_{[n-1]}-\bar{q}_{2} z_{n}^{2}+2 \delta_{n} \overline{\boldsymbol{\eta}}_{1}(\mathbf{z}) z_{n} \mathbf{z}_{[n-1]} \\
& +2 \delta_{n} \bar{\eta}_{2}(\mathbf{z}) z_{n}^{2} \\
= & -\mathbf{z}_{[n-1]}^{T} \overline{\mathbf{Q}}_{[n-1]} \mathbf{z}_{[n-1]}-2\left(\overline{\mathbf{q}}_{1}^{T}-\delta_{n} \overline{\boldsymbol{\eta}}_{1}(\mathbf{z})\right) z_{n} \mathbf{z}_{[n-1]}-\bar{q}_{2} z_{n}^{2}+2 \delta_{n} \bar{\eta}_{2}(\mathbf{z}) z_{n}^{2} \\
= & -\left\|\mathbf{z}_{[n-1]}+\overline{\mathbf{Q}}_{[n-1]}^{-1}\left(\overline{\mathbf{q}}_{1}^{T}-\delta_{n} \overline{\bar{\eta}}_{1}(\mathbf{z})\right) z_{n}\right\|_{\overline{\mathbf{Q}}_{[n-1]}^{2}-\left(\bar{q}_{2}-\overline{\mathbf{q}}_{1}^{T} \overline{\mathbf{Q}}_{[n-1]}^{-1} \overline{\mathbf{q}}_{1}\right) z_{n}^{2}} \\
& +2 \delta_{n} \bar{\eta}_{2}(\mathbf{z}) z_{n}^{2}-2 \delta_{n} \overline{\mathbf{q}}_{1} \overline{\mathbf{Q}}_{[n-1]} \overline{\boldsymbol{\eta}}_{(\mathbf{z}}(\mathbf{z}) z_{n}^{2}+\delta_{n}^{2} \overline{\bar{\eta}}_{1}(\mathbf{z}) \overline{\mathbf{Q}}_{[n-1]}^{-1} \overline{\boldsymbol{\eta}}_{1}^{T}(\mathbf{z}) z_{n}^{2}
\end{aligned}
$$

Define:

$$
\bar{q}_{0}(\mathbf{z})=\left\|\mathbf{z}_{[n-1]}+\overline{\mathbf{Q}}_{[n-1]}^{-1}\left(\overline{\mathbf{q}}_{1}^{T}-\delta_{n} \overline{\bar{\eta}}_{1}(\mathbf{z})\right) z_{n}\right\|_{\overline{\mathbf{Q}}_{[n-1]}}^{2}-\left(\bar{q}_{2}-\overline{\mathbf{q}}_{1}^{T} \overline{\mathbf{Q}}_{[n-1]}^{-1} \overline{\mathbf{q}}_{1}\right) z_{n}^{2}
$$

Here the first term in (216) is obviously positive definite and the second term is also positive, due to the positive definiteness of $\overline{\mathbf{Q}}$. Hence, the expression for $\dot{\bar{V}}$ in (208) can be written:

$$
\dot{\bar{V}}=\underbrace{-\bar{q}_{0}(\mathbf{z})+\left(\bar{r}^{-1}(\mathbf{z})-R^{-1}-\bar{\sigma}(\mathbf{z})\right) b_{n}^{2} \delta_{n}^{2} z_{n}^{2}}_{-\bar{q}(\mathbf{z})}-\bar{r}(\mathbf{z}) u^{2}
$$

where possible non-positive terms are collected into a new variable $\bar{\sigma}(\mathbf{z})$ which is:

$$
\bar{\sigma}(\mathbf{z})=2 b_{n}^{-2} \delta_{n}^{-1} \overline{\boldsymbol{\eta}}_{2}(\mathbf{z})-2 b_{n}^{-2} \delta_{n}^{-1} \overline{\mathbf{q}}_{1}^{T} \overline{\mathbf{Q}}_{[n-1]} \overline{\boldsymbol{\eta}}^{T}(\mathbf{z})+b_{n}^{-2} \overline{\boldsymbol{\eta}}_{1}(\mathbf{z}) \overline{\mathbf{Q}}_{[n-1]}^{-1} \overline{\boldsymbol{\eta}}_{1}^{T}(\mathbf{z})
$$


Choice of $\tilde{r}(\mathbf{z})$

For the control law

$$
u=-\overline{\boldsymbol{r}}^{-1}(\mathbf{z}) \mathbf{b}^{T} \Delta \mathbf{z}=-\overline{\boldsymbol{r}}^{-1}(\mathbf{z}) b_{n} \delta_{n} z_{n}
$$

several possible choices for $\bar{r}(\mathbf{z})$ that satisfies (211)-(213).

- One choice is

$$
\bar{r}(\mathbf{z})=\left(\left(R^{-1}+\bar{\sigma}(\mathbf{z})\right)^{2}+\bar{\varepsilon}(\mathbf{z})\right)^{-1 / 2}
$$

where $\bar{\varepsilon}(\mathbf{z})$ is positive definite and guarantees that $\bar{r}(\mathbf{0})=R$, for instance:

$$
\bar{\varepsilon}(\mathbf{z})=\lambda \frac{\bar{\sigma}^{2}(\mathbf{z})}{1+\bar{\sigma}^{2}(\mathbf{z})}, \quad \text { where } \lambda \varepsilon(0,1]
$$

- A leaner choice of $\bar{r}(\mathbf{z})$ is:

$$
\bar{r}(\mathbf{z})=\left\{\begin{array}{cl}
\left(R^{-1}+\bar{\sigma}(\mathbf{z})\right)^{-1} & : \quad \bar{\sigma}(\mathbf{z}) \geq 0 \\
R & : \quad \bar{\sigma}(\mathbf{z})<0
\end{array}\right.
$$

which dominates $R^{-1}+\bar{\sigma}(\mathbf{z})$ only when necessary.

\section{Inverse optimality}

From (208) it is seen that the control law (207) is inverse optimal with respect to the cost functional

which locally satisfies

$$
J=\int_{0}^{\infty}\left[\bar{q}(\mathbf{z})+\bar{r}(\mathbf{z}) u^{2}\right] d t
$$

$$
J_{1}=\int_{0}^{\infty}\left[\mathbf{z}^{T} \overline{\mathbf{Q}} \mathbf{z}+R u^{2}\right] d t
$$

which in original coordinates equals to (189).

\section{Extension to a larger class of nonlinear systems}

A straightforward extension of the LOB design to a state dependent $g_{n}(\mathbf{x})$, that is

$$
\dot{x}_{n}=f_{n}(\mathbf{x})+g_{n}(\mathbf{x}) \bar{u}
$$

where $g_{n}(\mathbf{x}) \neq 0, \forall \mathbf{x}$ and $g_{n}(0)=b_{n}$, can be found by defining the control input to be:

$$
u=\frac{b_{n}}{g_{n}(\mathbf{x})} \bar{u}
$$

In this case the resulting cost functional becomes

$$
J=\int_{0}^{\infty}\left[q(\mathbf{x})+r(\mathbf{x})\left(\frac{b_{n}}{g_{n}(\mathbf{x})}\right)^{2} u^{2}\right] d t
$$

\subsubsection{Recursive backstepping procedure}

\section{Linear backstepping}

Although the linear part $\mathbf{z}=\mathbf{L x}$ of the diffeomorphism is already known, the mapping in the framework of backstepping must be described in order to prepare for the nonlinear backstepping at the next stage. We first rewrite the linearized system (189) as 


$$
\begin{aligned}
\dot{x}_{i} & =\mathbf{a}_{[i]} \mathbf{x}_{[i]}+x_{i+1} \quad i=1 \ldots n-1 \\
\dot{x}_{n} & =\mathbf{a}_{[n]} \mathbf{x}_{[n]}+b_{n} \cdot u_{1}
\end{aligned}
$$

where $\mathbf{a}_{[i]}$ is the $i$-th row vector of $\mathbf{A}_{[i]}$ :

$$
\mathbf{a}_{[i]}=\left[a_{i 1}, \ldots, a_{i i}\right]
$$

Moreover, define the row vector:

$$
\bar{\alpha}_{[i]}=\mathbf{l}_{[i]} \mathbf{L}_{[i]}^{-1}
$$

where the row vectors $\mathbf{I}_{[i]}$ are extracted from $\mathbf{L}_{[i]}$ according to:

$$
\mathbf{I}_{[i]}=\left[l_{i 1}, \ldots, l_{i i}\right]
$$

for $i=1 \ldots n-1$. The error coordinates are given as

$$
\begin{aligned}
& z_{1}=x_{1} \\
& z_{i}=x_{i}-\mathbf{1}_{[i-1]} \mathbf{x}_{[i-1]}, \quad i=2 \ldots n
\end{aligned}
$$

which are given by the transformation $\mathbf{z}=\mathbf{L x}$, where $\mathbf{z}=\left[z_{1}, \ldots z_{n}\right]^{T}$.

Property 3.1. For $1 \leq k \leq n$ :

$$
\mathbf{z}_{[k]}=\mathbf{L}_{[k]} \mathbf{x}_{[k]} \text { where } \mathbf{L}_{[k]} \text { is invertible. }
$$

Property 3.2. For $1 \leq k<n$ :

$$
\dot{\mathbf{z}}_{[k]}=\overline{\mathbf{A}}_{[k]} \mathbf{z}_{[k]}+\left[\begin{array}{c}
\mathbf{0}_{[k-1]} \\
z_{k+1}
\end{array}\right]
$$

\section{Step 1}

The dynamics of $z_{1}$ is:

$$
\dot{z}_{1}=a_{11} z_{1}+x_{2}=\left(\mathbf{a}_{[1]}+\bar{\alpha}_{[1]}\right) \mathbf{z}_{[1]}+\left(x_{2}-\bar{\alpha}_{1}\right)
$$

where the stabilizing function $\bar{\alpha}_{1}\left(z_{1}\right)=\bar{\alpha}_{[1]} z_{1}$. Thus, the $z_{1}$-subsystem can be written as:

$$
\dot{z}_{1}=\overline{\mathbf{a}}_{[1]} \mathbf{z}_{[1]}+z_{2}, \quad \overline{\mathbf{a}}_{[1]}=\mathbf{a}_{[1]}+\bar{\alpha}_{[1]}
$$

\section{Step i}

Define $z_{i}=x_{i}-\bar{\alpha}_{i-1}\left(\mathbf{z}_{[i-1]}\right)$. The dynamics of $z_{i}$ are given by:

$$
\dot{z}_{i}=\mathbf{a}_{[i]} \mathbf{L}_{[i]}^{-1} \mathbf{z}_{[i]}+x_{i+1}-\frac{\partial \bar{\alpha}_{i-1}}{\partial \mathbf{z}_{[i-1]}}\left[\overline{\mathbf{A}}_{[i-1]} \mathbf{z}_{[i-1]}+\left[\begin{array}{c}
\mathbf{0}_{[i-2]} \\
z_{i}
\end{array}\right]\right]
$$

By selecting $\bar{\alpha}_{i}\left(\mathbf{z}_{[i]}\right)=\bar{\alpha}_{[i]} \mathbf{z}_{[i]}=\mathbf{l}_{[i]} \mathbf{L}_{[i]}{ }^{\prime} \mathbf{z}_{[i]}$ this simplifies to:

$$
\dot{z}_{i}=\overline{\mathbf{a}}_{[i]} \mathbf{z}_{[i]}+z_{i+1}
$$

where, by using Properties 3.1 and 3.2, yields:

$$
\overline{\mathbf{a}}_{[i]}=\mathbf{a}_{[i]} \mathbf{L}_{[i]}^{-1}+\bar{\alpha}_{[i]}-\left[\bar{\alpha}_{[i-1]} \overline{\mathbf{A}}_{[i-1]} \bar{\alpha}_{i-1, i-1}\right]
$$

Here $\bar{\alpha}_{i-1, i-1}$ is defined as the (i-1)-th element of $\bar{\alpha}_{[i-1]}$.

\section{Step n}

At the last step we define:

$$
z_{n}=x_{n}-\bar{\alpha}_{n-1}\left(\mathbf{z}_{[n-1}\right)
$$


where the dynamics is given as:

$$
\dot{z}_{n}=\mathbf{a}_{[n]} \mathbf{L}^{-1} \mathbf{z}+b_{n} u_{l}-\frac{\partial \bar{\alpha}_{n-1}}{\partial \mathbf{z}_{[n-1]}}\left[\overline{\mathbf{A}}_{[n-1]} \mathbf{z}_{[n-1]}+\left[\begin{array}{c}
\mathbf{0}_{[n-2]} \\
z_{n}
\end{array}\right]\right]
$$

which by using (233) yields the dynamics:

$$
\dot{z}_{n}=\overline{\mathbf{a}}_{[n]} \mathbf{z}+b_{n} u_{l}
$$

The linear system can now be written compactly as $\dot{\mathbf{z}}=\overline{\mathbf{A}} \mathbf{z}+\mathbf{b} u$ in the new coordinates.

\section{Nonlinear backstepping}

Now that the recursive steps of the linear backstepping are described, we are ready to perform the nonlinear backstepping. In the linear case the stabilizing functions were chosen as $\bar{\alpha}_{i}\left(\mathbf{z}_{[i]}\right)=\bar{\alpha}_{[i]} \mathbf{z}_{[i]}$. In the nonlinear case we add a nonlinear term, $\check{\alpha}_{i}\left(\mathbf{z}_{[i]}\right)$, to the linear one. For simplicity these nonlinear terms are selected to cancel the nonlinearities that appears at each step. However, this procedure can be modified such that "good" nonlinear damping terms are exploited. With the completion of each step a new state $z_{i}=\tilde{\phi}_{i}\left(\mathbf{x}_{i i]}\right)$ is defined. At the last step, the recursive backstepping creates a lower-triangular diffeomorphism $\mathbf{z}=\Phi(\mathbf{x})$ which can be separated into a linear term $\mathbf{L x}$ and a nonlinear term $\tilde{\Phi}(\mathbf{x})=\Phi(\mathbf{x})-\mathbf{L x}$.

\section{Step 1}

Let $z_{1}=x_{1}$. Thus

$$
\dot{z}_{1}=f_{1}\left(z_{1}\right)+x_{2}=a_{11} z_{1}+\check{f}_{1}\left(z_{1}\right)+x_{2}
$$

where $\check{f}_{1}\left(z_{1}\right)=f_{1}\left(z_{1}\right)-a_{11} z_{1}$ and $\check{f}_{1}(0)=0$. In the general setting this is written:

$$
\dot{z}_{1}=\mathbf{a}_{[1]} \mathbf{z}_{[1]}+\check{f}_{1}\left(\mathbf{z}_{[1]}\right)+x_{2}
$$

The stabilizing function is chosen as $\bar{\alpha}_{1}\left(\mathbf{z}_{[1]}\right)=\bar{\alpha}_{[1]} \mathbf{z}_{[1]}+\check{\alpha}_{1}\left(\mathbf{z}_{[1]}\right)$ where the first term is due to the linear backstepping and the latter term is a nonlinear term to be chosen. Now we get:

$$
\dot{z}_{1}=\mathbf{a}_{[1]} \mathbf{z}_{[1]}+\check{f}_{1}\left(\mathbf{z}_{[1]}\right)+\bar{\alpha}_{1}\left(\mathbf{z}_{[1]}\right)+\left(x_{2}-\bar{\alpha}_{1}\left(\mathbf{z}_{[1]}\right)\right)
$$

At each step we choose to cancel the nonlinear term, and at the first step this is obtained by $\check{\alpha}_{1}\left(\mathbf{z}_{[1]}\right)=-\breve{f}_{1}\left(\mathbf{z}_{[1]}\right)$. Thus, the second error variable is:

$$
z_{2}=x_{2}-\bar{\alpha}_{1}\left(\mathbf{z}_{[1]}\right)=x_{2}-\bar{\alpha}_{[1]} \mathbf{z}_{[1]}+\check{f}_{1}\left(\mathbf{z}_{[1]}\right)
$$

and

$$
\dot{z}_{1}=\overline{\mathbf{a}}_{[1]} \mathbf{z}_{[1]}+z_{2}
$$

\section{Step i}

Define $z_{i}=\phi_{i}\left(\mathbf{z}_{[i]}\right)=x_{i}-\bar{\alpha}_{i-1}\left(\mathbf{z}_{[i-1]}\right)$ where $\bar{\alpha}_{i-1}\left(\mathbf{z}_{[i-1]}\right)=\bar{\alpha}_{[i-1]} \mathbf{z}_{[i-1]}+\check{\alpha}_{[i-1]}$ $\left(\mathbf{z}_{[i-1]}\right)$. In the recursive steps we get:

$$
\dot{z}_{i}=\overline{\mathbf{a}}_{[i]} \mathbf{z}_{[i]}+\check{f}_{i}\left(\mathbf{z}_{[i]}\right)+\left(x_{i+1}-\bar{\alpha}_{i}\left(\mathbf{z}_{[i]}\right)\right.
$$

where $\overline{\mathbf{a}}_{[i]}$ is given by (233) and 


$$
\begin{aligned}
\check{f}_{i}\left(\mathbf{z}_{[i]}\right) & =\bar{\alpha}\left(\mathbf{z}_{[i]}\right)+\mathbf{a}_{[i]} \check{\Psi}_{[i]}\left(\mathbf{z}_{[i]}\right)+\check{f}_{i}\left(\Phi_{[i]}^{-1}\left(\mathbf{x}_{[i]}\right)\right) \\
& -\frac{\partial \check{\alpha}_{i-1}}{\partial \mathbf{z}_{[i-1]}}\left[\overline{\mathbf{A}}_{[i-1]} \mathbf{z}_{[i-1]}+\left[\begin{array}{c}
\mathbf{0}_{[i-2]} \\
z_{i}
\end{array}\right]\right]-\frac{\partial \bar{\alpha}_{i-1}}{\partial \mathbf{z}_{[i-1]}} \check{\mathbf{f}}_{[i-1]}\left(\mathbf{z}_{[i-1]}\right)
\end{aligned}
$$

and $\check{\Psi}_{[i]}\left(\mathbf{z}_{[i]}\right)=\Phi_{[i]}^{-1}\left(\mathbf{x}_{[i]}\right)-\mathbf{L}_{[i]}^{-1} \mathbf{z}_{[i]}$ contains only higher-order terms.

As in Step 1 we choose $\check{\alpha}_{i}\left(\mathbf{z}_{[i]}\right)$ to eliminate $\tilde{f}_{i}\left(\mathbf{z}_{[i]}\right)$ by:

$$
\begin{aligned}
\bar{\alpha}_{i}\left(\mathbf{z}_{[i]}\right) & =-\mathbf{a}_{[i]} \check{\Psi}_{[i]}\left(\mathbf{z}_{[i]}\right)+\check{f}_{i}\left(\Phi_{[i]}^{-1}\left(\mathbf{x}_{[i]}\right)\right) \\
& -\frac{\partial \check{\alpha}_{i-1}}{\partial \mathbf{z}_{[i-1]}}\left[\overline{\mathbf{A}}_{[i-1]} \mathbf{z}_{[i-1]}+\left[\begin{array}{c}
\mathbf{0}_{[i-2]} \\
z_{i}
\end{array}\right]\right]-\frac{\partial \bar{\alpha}_{i-1}}{\partial \mathbf{z}_{[i-1]}} \check{\mathbf{f}}_{[i-1]}\left(\mathbf{z}_{[i-1]}\right)
\end{aligned}
$$

such that

$$
\dot{z}_{i}=\overline{\mathbf{a}}_{[i]} \mathbf{z}_{[i]}+z_{i+1}
$$

where $\bar{\alpha}_{[i]}$ is given by (233).

\section{Step $n$}

At the final step we define $z_{n}=\phi_{n}(\mathbf{x})=x_{n}-\bar{\alpha}_{n-1}\left(\mathbf{z}_{[n-1]}\right)$ such that:

$$
\dot{z}_{n}=\overline{\mathbf{a}}_{[n]} \mathbf{z}+\check{f}_{n}(\mathbf{z})+b_{n} u
$$

where $\overline{\mathbf{a}}_{[n]}$ is given by (233) for $i=n$. Here:

$$
\begin{aligned}
\check{f}_{n}(\mathbf{z}) & =\mathbf{a}_{[n]} \check{\Psi}(\mathbf{z})+\check{f}_{i}\left(\Phi^{-1}(\mathbf{x})\right) \\
& -\frac{\partial \check{\alpha}_{n-1}}{\partial \mathbf{z}_{[n-1]}}\left[\overline{\mathbf{A}}_{[n-1]} \mathbf{z}_{[n-1]}+\left[\begin{array}{c}
\mathbf{0}_{[n-2]} \\
z_{n}
\end{array}\right]\right]-\frac{\partial \bar{\alpha}_{n-1}}{\partial \mathbf{z}_{[n-1]}} \check{\mathbf{f}}_{[n-1]}\left(\mathbf{z}_{[n-1]}\right)
\end{aligned}
$$

where $\check{\Psi}(\mathbf{z})=\Phi^{-1}(\mathbf{x})-\mathbf{L}^{-1} \mathbf{z}$ contains higher order terms. With this procedure the construction of the diffeomorphism $\mathbf{z}=\Phi(\mathbf{x})$ is completed and the original system (181) has been transformed into the form:

$$
\dot{\mathbf{z}}=\overline{\mathbf{A}} \mathbf{z}+\check{\mathbf{f}}(\mathbf{z})+\mathbf{b} u
$$

where

$$
\check{\mathbf{f}}(\mathbf{z})=\left[0,0 \ldots 0, \check{f}_{n}(\mathbf{z})\right]^{T}
$$

Example 3 (2nd-order nonlinear system) Consider the nonlinear system:

$$
\begin{gathered}
\dot{x}_{1}=x_{1}^{2}+x_{2} \\
\dot{x}_{2}=u
\end{gathered}
$$

In the linear case, the mapping to new state variables $\mathrm{z}$ is given by:

$$
\left[\begin{array}{l}
z_{1} \\
z_{2}
\end{array}\right]=\left[\begin{array}{cc}
1 & 0 \\
-l_{11} & 1
\end{array}\right]\left[\begin{array}{l}
x_{1} \\
x_{2}
\end{array}\right]
$$

Find the diffeomorphism $\mathrm{z}=\Phi(\mathbf{x})$ using backstepping. Write the transformed dynamics in new coordinates.

Solution. Let $z_{1}=\mathrm{x}_{1}$. Now:

$$
\dot{z}_{1}=\dot{x}_{1}=x_{1}^{2}+x_{2}=l_{11} z_{1}+\left(x_{2}+x_{1}^{2}-l_{11} z_{1}\right)
$$

Hence:

$$
z_{2}=x_{2}+x_{1}^{2}-l_{11} z_{1}
$$


and

$$
\Phi(\mathbf{x})=\left[\begin{array}{cc}
1 & 0 \\
-l_{11} & 1
\end{array}\right]\left[\begin{array}{l}
x_{1} \\
x_{2}
\end{array}\right]+\left[\begin{array}{c}
0 \\
x_{1}^{2}
\end{array}\right]
$$

Now:

Time differentiation of $z_{2}$ yields:

$$
\dot{z}_{1}=l_{11} z_{1}+z_{2}
$$

$$
\begin{aligned}
\dot{z}_{2} & =\dot{x}_{2}+2 z_{1} \dot{z}_{1}-l_{11} z_{1} \\
& =u+2 l_{11} z_{1}^{2}+2 z_{1} z_{2}-l_{11}^{2} z_{1}-l_{11} z_{2}
\end{aligned}
$$

Note that the nonlinear terms in $\dot{z}_{2}$ corresponds to $\breve{f}_{n}$ in (247).

\subsubsection{Summary}

The locally optimal procedure can be summarized as follows:

1. Given a nonlinear system in SISO strict feedback form:

$$
\dot{\mathbf{x}}=\mathbf{f}(\mathbf{x})+\mathbf{b} u
$$

Linearize it to:

$$
\dot{\mathbf{x}}=\mathbf{A x}+\mathbf{b} \boldsymbol{u}+\tilde{\mathbf{f}}(\mathbf{x})
$$

2. Given the linear cost functional:

$$
J_{l}=\int_{0}^{\infty}\left[\mathbf{x}^{T} \mathbf{Q} \mathbf{x}+R u_{l}^{2}\right] d t
$$

find the positive definite matrix $\mathbf{P}$ by solving the ARE.

3. Factorize $\mathbf{P}$ into:

$$
\mathbf{P}=\mathbf{L}^{T} \Delta \mathbf{L}
$$

using Cholesky factorization of $\mathbf{P}^{-1}$, and compute $\mathbf{L}$ and $\Delta$.

4. Find the diffeomorphism:

$$
\mathbf{z}=\boldsymbol{\Phi}(\mathbf{x})
$$

by recursive backstepping of the nonlinear system, as described in Section 3.1.5. The original system is now written in new coordinates

$$
\dot{\mathbf{z}}=\overline{\mathbf{A}} \mathbf{z}+\mathbf{b} \boldsymbol{u}+\check{\mathbf{f}}(\mathbf{z})
$$

where

$$
\check{f}(\mathbf{z})=\left[0,0, \ldots, 0, \check{f}_{n}(\mathbf{z})\right]^{T}
$$

and $\check{f}_{n}(\mathbf{z})$ is given by (247).

5. Factorize $\check{f}_{n}(\mathbf{z})$ into:

$$
\check{f}_{n}(\mathbf{z})=\overline{\boldsymbol{\eta}}_{1}(\mathbf{z}) \mathbf{z}_{[n-1]}+\bar{\eta}_{2}(\mathbf{z}) z_{n}
$$

6. Compute the analytic expression for:

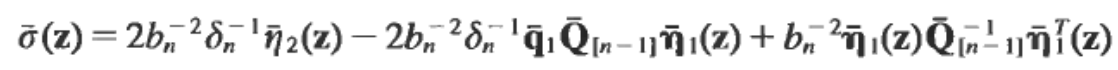

where $\overline{\mathbf{Q}}=\mathbf{L}^{-T} \mathbf{Q} \mathbf{L}^{-1}$. 
7. The control law is given by:

$$
u=-\overline{\boldsymbol{r}}^{-1}(\mathbf{z}) \mathbf{b}^{T} \Delta \mathbf{z}=-\overline{\boldsymbol{r}}^{-1}(\mathbf{z}) b_{n} \delta_{n} z_{n}
$$

where two possible choices for $\bar{r}^{-1}(z)$ are given in (218) and (220). The control law in $\mathbf{x}$-coordinates is:

$$
u=-\overline{\boldsymbol{r}}^{-1}(\boldsymbol{\Phi}(\mathbf{x})) \mathbf{b}^{T} \mathbf{L}^{-T} \mathbf{P} \mathbf{L}^{-1} \boldsymbol{\Phi}(\mathbf{x})
$$

\subsubsection{Example: Nonlinear Mass-Damper-Spring System}

Consider the 2nd-order system:

$$
\begin{aligned}
& \dot{x}_{1}=x_{2} \\
& \dot{x}_{2}=-k\left(x_{1}\right) x_{1}-d\left(x_{2}\right) x_{2}+u
\end{aligned}
$$

which is a mass-damper-spring system with nonlinear damping and nonlinear spring stiffness (for simplicity the mass, $m=1$ ). This system can be a moored ship moving in one degree of freedom, e.g., surge.

The linearized system is:

$$
\begin{aligned}
& \dot{x}_{1}=x_{2} \\
& \dot{x}_{2}=-k_{o} x_{1}-d_{o} x_{2}+u_{l}
\end{aligned}
$$

where

$$
k_{o}=\left.\frac{\partial k\left(x_{1}\right)}{\partial x_{1}}\right|_{x_{1}=0}, \quad d_{o}=\left.\frac{\partial d\left(x_{2}\right)}{\partial x_{2}}\right|_{x_{2}=0}
$$

Given a state cost matrix

$$
\mathbf{Q}=\left[\begin{array}{ll}
q_{11} & q_{12} \\
q_{12} & q_{22}
\end{array}\right]=\mathbf{Q}^{T}>\mathbf{0}
$$

and a scalar cost on control effort $R$ while P can be computed by solving the ARE (190). Next, $\mathbf{P}$ is written as $\mathbf{P}=\mathbf{L}^{T} \Delta \mathbf{L}$ where:

$$
\mathbf{L}=\left[\begin{array}{cc}
1 & 0 \\
-l_{11} & 1
\end{array}\right], \quad \Delta=\left[\begin{array}{rr}
\delta_{1} & 0 \\
0 & \delta_{2}
\end{array}\right]
$$

The original state vector $\mathbf{x}$ is mapped into $\mathbf{z}$-variables by $\mathbf{z}=\mathbf{L x}$, or:

$$
z_{1}=x_{1}, \quad z_{2}=x_{2}-l_{11} x_{1}
$$

The linear system in new coordinates becomes:

$$
\dot{\mathbf{z}}=\overline{\mathbf{A}} \mathbf{z}+\mathbf{b} u
$$

where

$$
\overline{\mathbf{A}}=\mathbf{L A L}^{-1}=\left[\begin{array}{cc}
l_{11} & 1 \\
-k_{o}-l_{11}-d_{o} l_{11} & -l_{11}-d_{o}
\end{array}\right]
$$

and

$$
\begin{aligned}
\overline{\mathbf{Q}} & =\mathbf{L}^{-T} \mathbf{Q} \mathbf{L}^{-1} \\
& =\left[\begin{array}{cc}
q_{11}+l_{11} q_{12}+\left(q_{12}+l_{11} q_{22}\right) l_{11} & q_{12}+l_{11} q_{22} \\
q_{12}+l_{11} q_{22} & q_{22}
\end{array}\right]
\end{aligned}
$$




$$
=\left[\begin{array}{ll}
\bar{q}_{11} & \bar{q}_{12} \\
\bar{q}_{12} & \bar{q}_{22}
\end{array}\right]
$$

\section{Backstepping}

Step 1: As in the linear case select $z_{1}=x_{1}$ such that:

$$
\dot{z}_{1}=\alpha_{11} z_{1}+\left(x_{2}-l_{11} z_{1}\right)=l_{11} z_{1}+z_{2}
$$

where the second variable is $z_{2}=x_{2}-l_{11} z_{1}$.

Step 2: Time differentiation of $z_{2}$ and substitution for $\dot{x}_{2}$ yields

$$
\dot{z}_{2}=\dot{x}_{2}-l_{11} \dot{z}_{1}=-k\left(z_{1}\right) z_{1}-d\left(x_{2}\right) x_{2}+u-l_{11}^{2} z_{1}-l_{11} z_{2}
$$

Now, substituting for $x_{2}=z_{2}+l_{11} z_{1}$, and adding and subtracting $k_{o} z_{1}, d_{o} l_{11} z_{1}$ and $d_{o} z_{1}$ to obtain $\bar{A}_{21} z_{1}$ and $\bar{A}_{22} z_{2}$, yields:

$$
\dot{z}_{2}=\left(-k_{o}-d_{o} l_{11}-l_{11}^{2}\right) z_{1}+\left(-l_{11}-d_{o}\right) z_{2}+u+\hat{f}_{2}(\mathbf{z})
$$

where $\hat{f}_{2}(\mathbf{z})$ can be written:

$$
\hat{f}_{2}(\mathbf{z})=\bar{\eta}_{1}\left(z_{1}, z_{2}\right) z_{1}+\bar{\eta}_{2}\left(z_{1}, z_{2}\right) z_{2}
$$

where

$$
\begin{aligned}
& \bar{\eta}_{1}\left(z_{1}, z_{2}\right)=\left[k_{o}-k\left(z_{1}\right)\right]+\left[d_{o}-d\left(z_{2}+l_{11} z_{1}\right)\right] l_{11} \\
& \bar{\eta}_{2}\left(z_{1}, z_{2}\right)=\left[d_{o}-d\left(z_{2}+l_{11} z_{1}\right)\right]
\end{aligned}
$$

which satisfies $\bar{\eta}_{1}(0,0)=0$ and $\bar{\eta}_{2}(0,0)=0$. Since the nonlinearities in this example appear in the last equation only, the diffeomorphism $\mathbf{z}=\boldsymbol{\Phi}(\mathbf{x})$ is equal to the linear mapping, i.e., $\mathbf{z}=\mathbf{L} \mathbf{x}$. The total system is:

$$
\dot{\mathbf{z}}=\overline{\mathbf{A}} \mathbf{z}+\mathbf{b} u+\hat{\mathbf{F}}(\mathbf{z}) \mathbf{z}
$$

where

$$
\hat{\mathbf{F}}(\mathbf{z})=\left[\begin{array}{cc}
0 & 0 \\
\bar{\eta}_{1}\left(z_{1}, z_{2}\right) & \bar{\eta}_{2}\left(z_{1}, z_{2}\right)
\end{array}\right]
$$

\section{Stability Analysis}

A Lyapunov function for the system is:

$$
\bar{V}=\mathbf{z}^{T} \Delta \mathbf{z}=\delta_{1} z_{1}^{2}+\delta_{2} z_{2}^{2}
$$

The LOB control law is:

$$
u=-\overline{\boldsymbol{r}}^{-1}(\mathbf{z}) \delta_{2} z_{2}
$$

where $\bar{r}(\mathbf{z})$ must satisfy:

$$
\bar{r}^{-1}(\mathrm{z}) \geq R^{-1}+\bar{\sigma}(\mathrm{z})
$$

and where $\bar{\sigma}(\mathbf{z})$ is given by (217) as:

$$
\bar{\sigma}(\mathbf{z})=2 \delta_{2}^{-1} \bar{\eta}_{2}(\mathbf{z})-2 \delta_{2}^{-1} \bar{q}_{12} \bar{q}_{11} \bar{\eta}_{1}(\mathbf{z})+\bar{q}^{-2} \bar{\eta}_{1}^{2}(\mathbf{z})
$$

This can be obtained by using one of the alternatives (218) or (220). Thus, $\dot{\bar{V}}$ can be written as:

$$
\dot{\bar{V}}=-\bar{q}(\mathbf{z})-\bar{r}(\mathbf{z}) u^{2}
$$


where

$$
\bar{q}(\mathbf{z})=\bar{q}_{o}(\mathbf{z})+\left(\bar{r}^{-1}(\mathbf{z})-R^{-1}-\bar{\sigma}(\mathbf{z})\right) \delta_{2}^{2} z_{2}^{2}
$$

and $\bar{q}_{o}(\mathbf{z})$ is given by (3.37) as:

$$
\bar{q}_{o}(\mathbf{z})=\left\|z_{1}+\bar{q}_{11}^{-1}\left(\bar{q}_{12}-\delta_{2} \bar{\eta}_{1}(\mathbf{z})\right) z_{2}\right\|_{q_{11}}^{2}+\left(\bar{q}_{22}-\bar{q}_{12}^{2} \bar{q}_{11}^{-1}\right) z_{2}^{2}
$$

The control law in original coordinates is:

$$
u=-\bar{r}^{-1}(\Phi(\mathbf{x})) \delta_{2}\left(x_{2}-l_{11} x_{1}\right)
$$

From $\dot{\bar{V}}$ we conclude that the system is GAS and LES. Moreover, both the optimality objectives $\mathrm{O} 1$ and $\mathrm{O} 2$ are satisfied.

Comment: Notice that the 2nd-order system in this example is equivalent to the autopilot example in Chapter 2.1 if:

$$
\begin{aligned}
k\left(x_{1}\right) & =0 \\
d\left(x_{2}\right) & =\frac{n_{3}}{T} x_{2}^{2}+\frac{n_{2}}{T} x_{2}+\frac{n_{1}}{T} \\
u & =K \delta
\end{aligned}
$$

and $n_{0}=0$.

\subsection{Locally Optimal and Robust Backstepping Design}

\subsubsection{Model Class}

A bounded unknown disturbance signal $\mathbf{w}(t, \mathbf{x}):[0, \infty) \times \mathfrak{R}^{n} \rightarrow \mathfrak{R}^{m}$ is added to the triangular system:

$$
\begin{aligned}
\dot{x}_{1}= & f_{1}\left(x_{1}\right)+x_{2}+\mathbf{h}_{1}^{T}\left(x_{1}\right) \mathbf{w} \\
\dot{x}_{2}= & f_{2}\left(x_{1}, x_{2}\right)+x_{3}+\mathbf{h}_{2}^{T}\left(x_{1}, x_{2}\right) \mathbf{w} \\
\dot{x}_{3}= & f_{3}\left(x_{1}, x_{2}, x_{3}\right)+x_{4}+\mathbf{h}_{3}^{T}\left(x_{1}, x_{2}, x_{3}\right) \mathbf{w} \\
& \vdots \\
\dot{x}_{n-1}= & f_{n-1}\left(x_{1}, x_{2}, \cdots, x_{n-1}\right)+x_{n}+\mathbf{h}_{n-1}^{T}\left(x_{1}, x_{2}, \cdots, x_{n-1}\right) \mathbf{w} \\
\dot{x}_{n}= & f_{n}\left(x_{1}, x_{2}, \cdots, x_{n}\right)+b_{n} \cdot u+\mathbf{h}_{n}^{T}\left(x_{1}, x_{2}, \cdots, x_{n}\right) \mathbf{w}
\end{aligned}
$$

which can be written compactly as:

$$
\dot{\mathbf{x}}=\mathbf{f}(\mathbf{x})+\mathrm{b} u+\mathbf{H}(\mathbf{x}) \mathbf{w}
$$

This system can be rewritten as:

$$
\dot{\mathbf{x}}=\mathbf{A} \mathbf{x}+\mathbf{b} \boldsymbol{u}+\mathbf{H}_{o} \mathbf{w}+\tilde{\mathbf{f}}(\mathbf{x})+\tilde{\mathbf{H}}(\mathbf{x}) \mathbf{w}
$$

where $\mathbf{A}$ and $\mathbf{b}$ are defined in Section 3.1 and

$$
\tilde{\mathbf{H}}(\mathbf{x})=\mathbf{H}(\mathbf{x})-\mathbf{H}_{o}, \quad \mathbf{H}_{o}=\mathbf{H}(\mathbf{0})
$$

The linear part of this system is:

$$
\dot{\mathbf{x}}=\mathbf{A} \mathbf{x}+\mathrm{bu}_{\imath}+\mathbf{H}_{o} \mathbf{w}_{l}
$$


It is assumed that $f_{i}, g_{i}$ and $h_{i}$ are sufficiently smooth, and that $f_{i}(0)=0$. In addition, let $\mathbf{h}_{o i}=\mathbf{h}_{i}(\mathbf{0}) \in \mathfrak{R}^{1 \times m}$.

\subsubsection{Control Objectives}

OR1: Global Objective. The ultimate control objective is to design a globally asymptomatically stabilizing feedback control law $u=\mu(\mathbf{x})$ which minimizes the cost functional:

$$
J=\int_{0}^{\infty}\left[q(\mathbf{x})+r(\mathbf{x}) u^{2}-\gamma^{2} \mathbf{w}^{T} \mathbf{w}\right] d t
$$

subject to the system dynamics:

$$
\dot{\mathbf{x}}=\mathbf{f}(\mathbf{x})+\mathbf{b} u+\mathbf{H}(\mathbf{x}) \mathbf{w}
$$

The desired disturbance attenuation level is represented by the scalar $\gamma>0$. As in the previous section it will be required that:

$$
q(\mathbf{x}) \text { is positive definite, and } r(\mathbf{x})>0 \quad \forall \mathbf{x}
$$

OR2: Local Objective. The second control objective is to find a control law that minimizes the cost functional:

$$
J_{l}=\int_{0}^{\infty}\left[\mathbf{x}^{T} \mathbf{Q} \mathbf{x}+R u_{l}^{2}-\gamma^{2} \mathbf{w}_{l}^{T} \mathbf{w}_{l}\right] d t
$$

locally, i.e. near the origin $\mathbf{x}=\mathbf{0}$, subject to the linearized system dynamics:

$$
\dot{\mathbf{x}}=\mathbf{A x}+\mathbf{b} u_{l}+\mathbf{H}_{o} \mathbf{w}_{l}
$$

As in the previous section it will be required that $q(\mathbf{x})$ and $r(\mathbf{x})$ in OR1 satisfies the local objectives:

$$
\begin{aligned}
r(\mathbf{0}) & =R \\
q_{x x}(\mathbf{0}) & =\left.\frac{1}{2} \frac{\partial^{2} q(x)}{\partial \mathbf{x}^{2}}\right|_{\mathrm{x}=0}=\mathbf{Q}
\end{aligned}
$$

\subsubsection{Locally Optimal and Robust Backstepping}

A linear control law $u_{l}$ that meets the local control objective $\mathrm{O} 2$ for the worst-case disturbance can easily be found by solving the generalized algebraic Riccati equation (GARE):

$$
\mathbf{P A}+\mathbf{A}^{T} \mathbf{P}+\mathbf{P}\left(\frac{1}{\gamma^{2}} \mathbf{H}_{o} \mathbf{H}_{o}^{T}-\mathbf{b} R^{-1} \mathbf{b}^{T}\right) \mathbf{P}+\mathbf{Q}=\mathbf{0}
$$

where $\mathbf{P}=\mathbf{P}^{T}>\mathbf{0}$. This solution exists for all $\gamma>\gamma^{*}>0$ assuming that $(\mathbf{A}, \mathbf{b})$ is controllable and $(\mathbf{A}, \mathbf{Q})$ is observable. Here $\gamma^{*}$ denotes the optimal disturbance attenuation level and $\gamma$ the desired attenuation level. The linear $\mathscr{H}_{\infty}$-controller is:

$$
\boldsymbol{u}_{l}=-\boldsymbol{R}^{-1} \mathbf{b}^{T} \mathbf{P} \mathbf{x}
$$

By factorization of $\mathbf{P}$ as in Section 3.1 the linear system under the transformation $\mathbf{z}=\mathbf{L x}$ becomes:

$$
\dot{\mathbf{z}}=\overline{\mathbf{A}} \mathbf{z}+\mathbf{b} u_{l}+\overline{\mathbf{H}}_{o} \mathbf{w}_{l}
$$




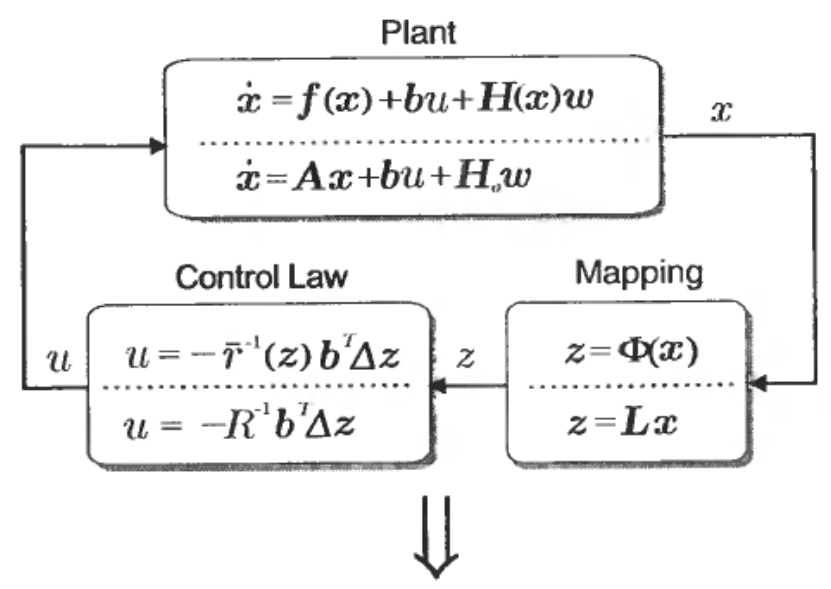

Cost functional

$$
\begin{aligned}
& J=\int_{0}^{\infty}\left[q(\boldsymbol{x})+r(\boldsymbol{x}) u^{2}-\boldsymbol{\gamma}^{2} \boldsymbol{w}^{T} \boldsymbol{w}\right] d t \\
& J=\int_{0}^{\infty}\left[\boldsymbol{x}^{T} \boldsymbol{Q} \boldsymbol{x}+R u^{2}-\boldsymbol{\gamma}^{2} \boldsymbol{w}^{T} \boldsymbol{w}\right] d t
\end{aligned}
$$

Figure 11. Relationship between linear $\mathscr{H}_{\infty}$-control (lower blocks) and nonlinear optimal and robust backstepping (upper blocks).

where $\overline{\mathbf{H}}_{o}=\mathbf{L}^{-1} \mathbf{H}_{o}$. Similarly the GARE (289) can be transformed to:

$$
\overline{\mathbf{A}}^{T} \Delta+\Delta \overline{\mathbf{A}}+\Delta\left(\frac{1}{\gamma^{2}} \breve{\mathbf{H}}_{o} \overline{\mathbf{H}}_{o}^{T}-\mathbf{b} R^{-1} \mathbf{b}^{T}\right) \Delta+\overline{\mathbf{Q}}=\mathbf{0}
$$

As in the previous section the diffeomorphism $\mathbf{z}=\Phi(\mathbf{x})$ is constructed by recursive backstepping. In new coordinates the nonlinear system (286) becomes:

$$
\dot{\mathbf{z}}=\overline{\mathbf{A}} \mathbf{z}+\mathbf{b} u+\breve{\mathbf{f}}(\mathbf{z})+\overline{\mathbf{H}}(\mathbf{z}) \mathbf{w}
$$

where $\check{\mathbf{f}}(\mathbf{z})$ and $\overline{\mathbf{H}}(\mathbf{z})$ are constructed during the steps of backstepping. The final control law is:

$$
u=-\bar{r}^{-1}(\mathbf{z}) \mathbf{b}^{T} \Delta \mathbf{z}=-\bar{r}^{-1}(\mathbf{z}) b_{n} \delta_{n} z_{n}
$$

where

$$
\begin{aligned}
\bar{r}^{-1}(\mathbf{z}) & \geq R^{-1}+\bar{\sigma}(\mathbf{z}) \\
\bar{r}(\mathbf{0}) & =R \\
\bar{r}(\mathbf{z}) & >0 \quad \forall \mathbf{z}
\end{aligned}
$$

which can be achieved by any of the choices for $\bar{r}^{-1}(z)$ in (218) or (220). If

$$
\bar{V}=\mathbf{z}^{T} \Delta \mathbf{z}
$$

is a Lyapunov function for the nonlinear system (286), $\dot{\bar{V}}$ can be written as:

$$
\dot{\bar{V}}=-\bar{q}(\mathbf{z})-\bar{r}(\mathbf{z}) u^{2}-\gamma^{2} \mathbf{w}^{T} \mathbf{w}
$$

which demonstrates that the inverse optimal objective OR1 is satisfied. 


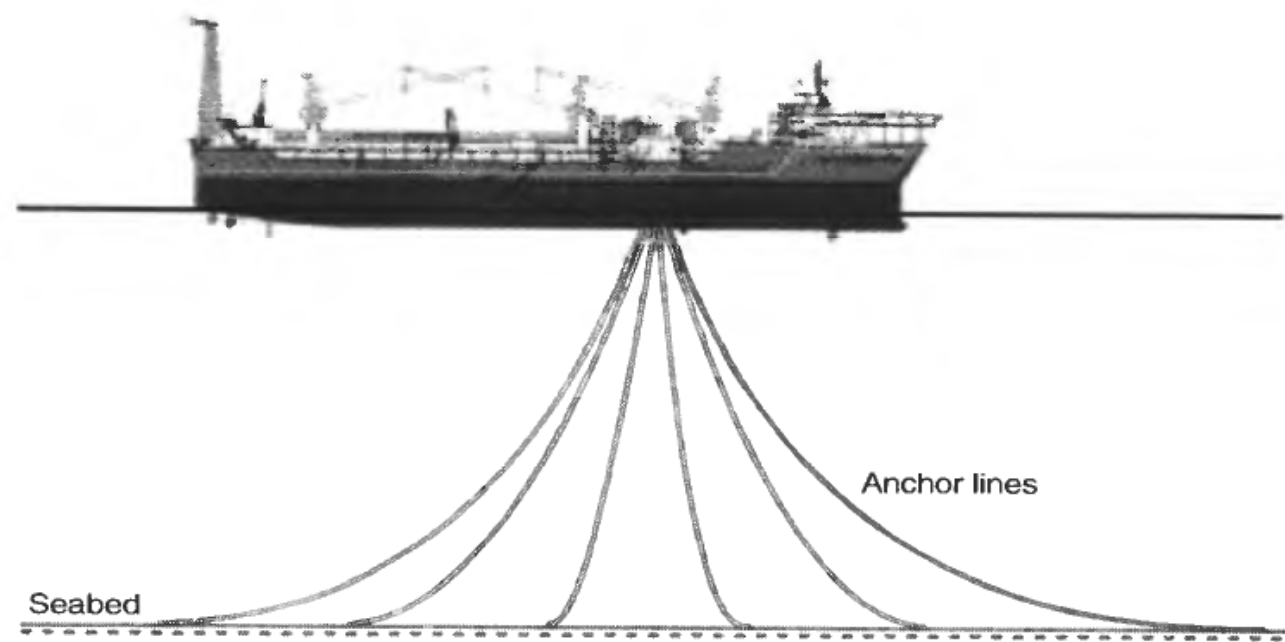

Figure 12. Ship and spread mooring system.

Thus, the only difference between the LOB design in Section 3.1 and the robust LOB is that here we solve the GARE instead of the ARE, and that the contents of $\mathbf{f}(\mathbf{z})$ and $\bar{\sigma}(\mathbf{z})$ takes into account the additional disturbances.

\subsubsection{Recursive Backstepping Procedure}

The linear part of the backstepping is equivalent to the procedure in Section 3.1.5. However, due to the additional disturbances in the system, the recursive backstepping procedure in this case becomes more elaborate. The interested reader is referred to Ezal, Pan and Kokotović (1997) or Ezal (1998) for more details. The locally optimal and robust backstepping design will be demonstrated on a ship control design example in the forthcoming section.

\subsection{Locally Optimal Control of Moored Ships}

The locally optimal and robust backstepping designs can be extended to MIMO systcms in block-strict-feedback form. In this section the robust LOB design is applied to ship control. The results presented here are based on a paper by Strand $e$ t al. (1998).

The ship model (148)-(149) in Section 2.2 can be extended to moored ships by adding a restoring term in the model. The deviation from the desired position and heading $\eta_{d}=\left[x_{d}, y_{d}, \psi_{d}\right]^{T}$ decomposed in vessel parallel coordinates is:

$$
\mathbf{e}=\mathbf{J}_{d}^{T}\left(\psi_{d}\right)\left(\boldsymbol{\eta}-\boldsymbol{\eta}_{d}\right)
$$

where

$$
\mathbf{J}_{d}\left(\psi_{d}\right)=\left[\begin{array}{ccc}
\cos \psi_{d} & -\sin \psi_{d} & 0 \\
\sin \psi_{d} & \cos \psi_{d} & 0 \\
0 & 0 & 1
\end{array}\right], \mathbf{J}_{e}(\tilde{\psi}) \wedge\left[\begin{array}{ccc}
\cos \tilde{\psi} & -\sin \tilde{\psi} & 0 \\
\sin \psi & \cos \psi & 0 \\
0 & 0 & 1
\end{array}\right]
$$

and $\tilde{\psi}=\psi-\psi_{d}$. In this example regulation is considered, such that $\boldsymbol{\eta}_{d} \equiv$ constant. The ship model can be formulated as:

$$
\begin{gathered}
\dot{\mathbf{e}}=\mathbf{J}_{e}(\tilde{\psi}) \boldsymbol{\nu} \\
\mathbf{M} \dot{\boldsymbol{\nu}}+\mathbf{D}(\boldsymbol{\nu}) \boldsymbol{\nu}+\mathbf{C}(\boldsymbol{\nu}) \boldsymbol{\nu}+\mathbf{G}(\mathbf{e}) \mathbf{e}=\overline{\mathbf{u}}+\mathbf{J}_{e}^{T}(\tilde{\psi}) \mathbf{w}
\end{gathered}
$$


where $\mathbf{G ( e ) e ~ i s ~ a ~ n o n l i n e a r ~ r e s t o r i n g ~ t e r m , ~ d u e ~ t o ~ t h e ~ m o o r i n g ~ s y s t e m . ~ I n ~ d y n a m i c ~}$ positioning $\mathbf{G}(\mathbf{e}) \equiv \mathbf{0}$. This system can be linearized about the origin $\mathbf{e}=\mathbf{0}, \boldsymbol{\nu}=\mathbf{0}$ such that:

$$
\mathbf{J}_{e}(0)=\mathbf{I}, \quad \mathbf{D}(\mathbf{0})=\mathbf{D}_{o}, \quad \mathbf{C}(\mathbf{0})=\mathbf{0}, \quad \mathbf{G}(\mathbf{0})=\mathbf{G}_{o}
$$

Hence, the linear system becomes:

$$
\begin{aligned}
& \dot{\mathbf{e}}=\boldsymbol{\nu} \\
& \dot{\boldsymbol{\nu}}=-\mathbf{M}^{-1} \mathbf{D}_{o} \boldsymbol{v}-\mathbf{M}^{-1} \mathbf{G}_{o} \mathbf{e}+\mathbf{M}^{-1} \overline{\mathbf{u}}_{l}+\mathbf{w}_{l}
\end{aligned}
$$

or compactly written:

$$
\dot{\mathbf{x}}=\mathbf{A x}+\mathbf{B} \overline{\mathbf{u}}_{l}+\mathbf{H}_{o} \mathbf{w}_{l}
$$

where $\mathbf{x}=\left[\mathbf{e}^{T}, \boldsymbol{\nu}^{T}\right]^{T}$ with obvious choice of $\mathbf{A}, \mathbf{B}$ and $\mathbf{H}_{o}$. Similarly, the nonlinear system (299)- (300) can be formulated as:

$$
\dot{\mathbf{x}}=\mathbf{f}(\mathbf{x})+\mathbf{B} \overline{\mathbf{u}}+\mathbf{H}(\mathbf{x}) \mathbf{w}
$$

The control objective is to find an inverse optimal control law that minimizes the cost functional

$$
J=\int_{0}^{t}\left[q(\mathbf{x})+\overline{\mathbf{u}}^{T} \mathbf{R}_{*}(\mathbf{x}) \overline{\mathbf{u}}-\gamma^{2} \mathbf{w}^{T} \mathbf{w}\right] d t
$$

where

$$
q(\mathbf{x}) \text { is positive definite and } \mathbf{R}_{*}(\mathbf{x})=\mathbf{R}_{*}^{T}(\mathbf{x})>0, \quad \forall \mathbf{x}
$$

The local optimal objective requires that:

$$
\mathbf{R}_{*}(\mathbf{0})=\mathbf{R}, \quad q_{x x}(\mathbf{0})=\frac{1}{2} \frac{\partial^{2} q(\mathbf{x})}{\partial \mathbf{x}^{2}}(\mathbf{0})=\mathbf{Q}
$$

where $\mathbf{Q}=\mathbf{Q}^{T}>0$ and $\mathbf{R}=\mathbf{R}^{T}>0$ are design cost matrices.

Since the ship model is in MIMO strict feedback form, the design methodology is quite similar to the LOB for SISO systems. A positive definite matrix $\mathbf{P}=\mathbf{P}^{T}>0$ can be found by solving the GARE:

$$
\mathbf{P A}+\mathbf{A}^{T} \mathbf{P}+\mathbf{P}\left(\frac{1}{\gamma^{2}} \mathbf{H}_{o} \mathbf{H}_{o}^{T}-\mathbf{B R}^{-1} \mathbf{B}^{T}\right) \mathbf{P}+\mathbf{Q}=\mathbf{0}
$$

As in the SISO case $\mathbf{P}$ can be factored into

$$
\mathbf{P}=\mathbf{L}^{T} \Delta \mathbf{L}
$$

where the block diagonal entries of $\mathbf{L}$ consists of identity matrices and the block diagonal entries of $\Delta$ contains positive definite sub-matrices $\Delta_{i}$. In this particular case we have:

$$
\mathbf{L}=\left[\begin{array}{cc}
\mathbf{I} & \mathbf{0} \\
-\mathbf{L}_{11} & \mathbf{I}
\end{array}\right], \quad \Delta=\left[\begin{array}{rr}
\Delta_{1} & \mathbf{0} \\
\mathbf{0} & \Delta_{2}
\end{array}\right]
$$

where all sub-matrices are of dimension $3 \times 3$, and $\Delta_{1}, \Delta_{2}$ are positive definite. Using the transformation $\mathbf{z}=\mathbf{L x}$ the linear part of the system (303) becomes: 


$$
\dot{\mathbf{z}}=\overline{\mathbf{A}} \mathbf{z}+\mathbf{B} \overline{\mathbf{u}}+\overline{\mathbf{H}}_{v} \mathbf{w}
$$

and

$$
\overline{\mathbf{A}}=\mathbf{L A L}^{-1}=\left[\begin{array}{cc}
\overline{\mathbf{A}}_{11} & \mathbf{I} \\
\overline{\mathbf{A}}_{21} & \overline{\mathbf{A}}_{22}
\end{array}\right], \quad \overline{\mathbf{H}}_{o}=\mathbf{L}^{-1} \mathbf{H}_{o}
$$

where

$$
\begin{aligned}
& \overline{\mathbf{A}}_{11}=\mathbf{L}_{11}, \\
& \overline{\mathbf{A}}_{21}=-\mathbf{M}^{-1} \mathbf{D}_{o} \mathbf{L}_{11}-\mathbf{M}^{-1} \mathbf{G}_{o}-\mathbf{L}_{11}^{2}, \\
& \overline{\mathbf{A}}_{22}=-\mathbf{M}^{-1} \mathbf{D}_{o}-\mathbf{L}_{11} .
\end{aligned}
$$

As in the previous sections the GARE (306) can be transformed into

$$
\overline{\mathbf{A}}^{T} \Delta+\Delta \overline{\mathbf{A}}+\Delta\left(\frac{1}{\gamma^{2}} \overline{\mathbf{H}}_{o} \overline{\mathbf{H}}_{o}^{T}-\mathbf{B R}^{-1} \mathbf{B}^{T}\right) \Delta+\overline{\mathbf{Q}}=\mathbf{0}
$$

where $\overline{\mathbf{Q}}=\mathbf{L}^{-T} \mathbf{Q} \mathbf{L}^{-1}=\overline{\mathbf{Q}}^{T}>\mathbf{0}$.

\section{Nonlinear Backstepping}

The nonlinear system is transformed into new coordinates by backstepping.

Step 1: Let $\mathbf{z}_{1}=\mathbf{e}$. Hence

$$
\dot{\mathbf{z}}_{1}=\mathbf{J}_{e} \boldsymbol{\nu}=\overline{\boldsymbol{\alpha}}_{1}\left(\mathbf{z}_{1}\right)+\left(\mathbf{J}_{e} \boldsymbol{\nu}-\overline{\boldsymbol{\alpha}}_{1}\left(\mathbf{z}_{1}\right)\right)
$$

where

$$
\mathbf{z}_{2}=\mathbf{J}_{e} \boldsymbol{\nu}-\overline{\boldsymbol{\alpha}}_{1}\left(\mathbf{z}_{1}\right)
$$

Thus:

$$
\dot{\mathbf{z}}_{1}=\mathbf{L}_{11} \mathbf{z}_{1}+\mathbf{z}_{2}
$$

Now, the stabilizing vector fields are chosen as $\overline{\boldsymbol{\alpha}}_{1}\left(\mathbf{z}_{1}\right)=\mathbf{L}_{11} \mathbf{z}_{1}$.

Step 2: From (312) we get:

$$
\dot{\mathbf{z}}_{2}=\mathbf{J}_{e} \dot{\boldsymbol{\nu}}+\dot{\mathbf{J}}_{e} \boldsymbol{\nu}-\mathbf{L}_{11} \dot{\mathbf{z}}_{1}+\mathbf{J}_{e} \mathbf{M}^{-1} \mathbf{J}_{e}^{T} \mathbf{w}
$$

at which we substitute the ship model (300) for $\dot{\nu}$ and get:

$$
\begin{aligned}
\dot{\mathbf{z}}_{2}= & -\mathbf{J}_{e} \mathbf{M}^{-1} \mathbf{D}(\boldsymbol{\nu}) \boldsymbol{\nu}-\mathbf{J}_{e} \mathbf{M}^{-1} \mathbf{C}(\boldsymbol{\nu}) \boldsymbol{\nu}-\mathbf{J}_{e} \mathbf{M}^{-1} \mathbf{G}\left(\mathbf{z}_{1}\right) \mathbf{z}_{1} \\
& +\mathbf{J}_{e} \mathbf{M}^{-1} \overline{\mathbf{u}}+\dot{\mathbf{J}}_{e} \boldsymbol{\nu}-\mathbf{L}_{11}^{2} \mathbf{z}_{1}-\mathbf{L}_{11} \mathbf{z}_{2}+\mathbf{J}_{e} \mathbf{M}^{-1} \mathbf{J}_{e}^{T} \mathbf{W}
\end{aligned}
$$

Using $\mathbf{J}_{e} \boldsymbol{\nu}=\mathbf{z}_{2}+\mathbf{L}_{11} \mathbf{z}_{1}$, yields:

$$
\begin{aligned}
\dot{\mathbf{z}}_{2}= & -\mathbf{J}_{e} \mathbf{M}^{-1} \mathbf{D}(\boldsymbol{\nu}) \mathbf{J}_{e}^{T} \mathbf{L}_{11} \mathbf{z}_{1}-\mathbf{J}_{e} \mathbf{M}^{-1} \mathbf{D}(\boldsymbol{\nu}) \mathbf{J}_{e}^{T} \mathbf{z}_{2} \\
& -\mathbf{J}_{e} \mathbf{M}^{-1} \mathbf{C}(\boldsymbol{\nu}) \mathbf{J}_{e}^{T} \mathbf{L}_{11} \mathbf{z}_{1}-\mathbf{J}_{e} \mathbf{M}^{-1} \mathbf{C}(\boldsymbol{\nu}) \mathbf{J}_{e}^{T} \mathbf{z}_{2} \\
& -\mathbf{J}_{e} \mathbf{M}^{-1} \mathbf{G}\left(\mathbf{z}_{1}\right) \mathbf{z}_{1}+\dot{\mathbf{J}}_{e} \mathbf{J}_{e}^{T} \mathbf{L}_{11} \mathbf{z}_{1}+\mathbf{J}_{e} \mathbf{J}_{e}^{T} \mathbf{z}_{2} \\
& +\mathbf{J}_{e} \mathbf{M}^{-1} \overline{\mathbf{u}}-\mathbf{L}_{11}^{2} \mathbf{z}_{1}-\mathbf{L}_{11} \mathbf{z}_{2}+\mathbf{J}_{e} \mathbf{M}^{-1} \mathbf{J}_{e}^{T} \mathbf{w}
\end{aligned}
$$

By adding and subtracting $\overline{\mathbf{A}}_{21} \mathbf{z}_{1}$ and $\overline{\mathbf{A}}_{22} \mathbf{z}_{2}$ we get:

$$
\dot{\mathbf{z}}_{2}=\overline{\mathbf{A}}_{21} \mathbf{z}_{1}+\overline{\mathbf{A}}_{22} \mathbf{z}_{2}+\overline{\mathbf{N}}_{1}(\mathbf{z}) \mathbf{z}_{1}+\overline{\mathbf{N}}_{2}(\mathbf{z}) \mathbf{z}_{2}+\mathbf{J}_{e} \mathbf{M}^{-1} \overline{\mathbf{u}}+\mathbf{J}_{e} \mathbf{M}^{-1} \mathbf{J}_{e}^{T} \mathbf{W}
$$


where $\mathbf{z}=\left[\mathbf{z}_{1}^{T}, \mathbf{z}_{2}^{T}\right]^{T}$ and

$$
\begin{aligned}
\overline{\mathbf{N}}_{1}(\mathbf{z})= & -\mathbf{J}_{e} \mathbf{M}^{-1} \mathbf{D}(\boldsymbol{\nu}) \mathbf{J}_{e}^{T} \mathbf{L}_{0}+\mathbf{M}^{-1} \mathbf{D} \mathbf{L}_{0} \\
& -\mathbf{M}^{-1} \mathbf{G}\left(\mathbf{z}_{1}\right)+\mathbf{M}^{-1} \mathbf{G} \\
& -\mathbf{J}_{e} \mathbf{M}^{-1} \mathbf{C}(\boldsymbol{\nu}) \mathbf{J}_{e}^{T} \mathbf{L}_{0}+\mathbf{J}_{e} \mathbf{J}_{e}^{T} \mathbf{L}_{11}, \\
\overline{\mathbf{N}}_{2}(\mathbf{z})= & -\mathbf{J}_{e} \mathbf{M}^{-1} \mathbf{D}(\nu) \mathbf{J}_{e}^{T}+\mathbf{M}^{-1} \mathbf{D} \\
& -\mathbf{J}_{e} \mathbf{M}^{-1} \mathbf{C}(\nu) \mathbf{J}_{e}^{T}+\dot{\mathbf{J}}_{e} \mathbf{J}_{e}^{T}
\end{aligned}
$$

which satisfies $\overline{\mathbf{N}}_{1}(\mathbf{0})=\mathbf{0}$ and $\overline{\mathbf{N}}_{2}(\mathbf{0})=\mathbf{0}$. The transformed system can be written:

$$
\dot{\mathbf{z}}=\overline{\mathbf{A}} \mathbf{z}+\mathbf{B}(\mathbf{z}) \overline{\mathbf{u}}+\mathbf{N}(\mathbf{z}) \mathbf{z}+\overline{\mathbf{H}}(\mathbf{x}) \mathbf{w}
$$

where

$$
\mathbf{B}(\mathbf{z})=\left[\begin{array}{c}
\mathbf{0} \\
\mathbf{J}_{e} \mathbf{M}^{-1}
\end{array}\right], \quad \mathbf{N}(\mathbf{z})=\left[\begin{array}{cc}
\mathbf{0} & \mathbf{0} \\
\overline{\mathbf{N}}_{1}(\mathbf{z}) & \overline{\mathbf{N}}_{2}(\mathbf{z})
\end{array}\right]
$$

\section{Locally Optimal and Robust Backstepping}

Let:

$$
\bar{V}=\mathbf{z}^{T} \Delta \mathbf{z}=\mathbf{z}_{1}^{T} \Delta_{1} \mathbf{z}_{1}+\mathbf{z}_{2}^{T} \Delta_{2} \mathbf{z}_{2}
$$

be a Lyapunov function for the system (317). Thus:

$$
\begin{aligned}
\dot{\bar{V}}= & \mathbf{z}^{T}\left(\overline{\mathbf{A}}^{T} \Delta+\Delta \overline{\mathbf{A}}\right) \mathbf{z}+2 \overline{\mathbf{u}}^{T} \mathbf{M}^{-1} \mathbf{J}_{e}^{T} \Delta_{2} \mathbf{z}_{2} \\
& +2 \mathbf{w}^{T} \mathbf{J}_{e} \mathbf{M}^{-1} \mathbf{J}_{e}^{T} \Delta_{2} \mathbf{z}_{2}+2 \mathbf{w}^{T} \mathbf{J}_{e} \mathbf{M}^{-1} \mathbf{J}_{e}^{T} \Delta_{2} \mathbf{z}_{2} \\
& +2 \mathbf{z}_{2}^{T} \Delta_{2} \overline{\mathbf{N}}_{1}(\mathbf{z}) \mathbf{z}_{1}+2 \mathbf{z}_{2}^{T} \Delta_{2} \overline{\mathbf{N}}_{2}(\mathbf{z}) \mathbf{z}_{2} .
\end{aligned}
$$

By using the GARE (310) and the identities:

$$
\begin{gathered}
2 \overline{\mathbf{u}}^{T} \mathbf{M}^{-1} \mathbf{J}_{e}^{T} \Delta_{2} \mathbf{z}_{2} \equiv-\overline{\mathbf{u}}^{T} \overline{\mathbf{R}}_{*}(\mathbf{z}) \overline{\mathbf{u}}+\left\|\overline{\mathbf{u}}+\overline{\mathbf{R}}_{*}^{-1}(\mathbf{z}) \mathbf{M}^{-1} \mathbf{J}_{e}^{T} \Delta_{2} \mathbf{z}_{2}\right\|_{\overline{\mathbf{R}}_{\bullet}(\mathbf{z})}^{2} \\
-\mathbf{z}_{2}^{T} \Delta_{2} \mathbf{J}_{e} \mathbf{M}^{-1} \overline{\mathbf{R}}_{*}^{-1}(\mathbf{z}) \mathbf{M}^{-1} \mathbf{J}_{e}^{T} \Delta_{2} \mathbf{z}_{2}, \\
2 \mathbf{w}^{T} \mathbf{J}_{e} \mathbf{M}^{-1} \mathbf{J}_{e}^{T} \Delta_{2} \mathbf{z}_{2} \equiv \gamma^{2} \mathbf{w}^{T} \mathbf{w}+\frac{1}{\gamma^{2}} \mathbf{z}_{2}^{T} \Delta_{2} \mathbf{J}_{e} \mathbf{M}^{-2} \mathbf{J}_{e}^{T} \Delta_{2} \mathbf{z}_{2} \\
-\gamma^{2}\left\|\mathbf{w}-\frac{1}{\gamma^{2}} \mathbf{J}_{e} \mathbf{M}^{-1} \mathbf{J}_{e}^{T} \Delta_{2} \mathbf{z}_{2}\right\|^{2}
\end{gathered}
$$

we get:

$$
\begin{aligned}
\dot{\bar{V}}= & -\mathbf{z}^{T} \overline{\mathbf{Q}} \mathbf{z}-\overline{\mathbf{u}}^{T} \overline{\mathbf{R}}_{*}(\mathbf{z}) \overline{\mathbf{u}}+\gamma^{2} \mathbf{w}^{T} \mathbf{w}+\left\|\overline{\mathbf{u}}+\overline{\mathbf{R}}_{*}^{-1} \mathbf{M}^{-1} \mathbf{J}_{e}^{T} \Delta_{2} \mathbf{z}_{2}\right\|_{\overline{\mathbf{R}}_{*}(\mathbf{z})}^{2} \\
& -\gamma^{2}\left\|\mathbf{w}-\frac{1}{\gamma^{2}} \mathbf{J}_{e} \mathbf{M}^{-1} \mathbf{J}_{e}^{T} \Delta_{2} \mathbf{z}_{2}\right\|^{2}+\mathbf{z}_{2}^{T} \Delta_{2} \mathbf{M}^{-1} \mathbf{R}^{-1} \mathbf{M}^{-1} \Delta_{2} \mathbf{z}_{2} \\
& -\frac{1}{\gamma^{2}} \mathbf{z}_{2}^{T} \Delta_{2} \mathbf{M}^{-2} \Delta_{2} \mathbf{z}_{2}+\frac{1}{\gamma^{2}} \mathbf{z}_{2}^{T} \Delta_{2} \mathbf{J}_{e} \mathbf{M}^{-2} \mathbf{J}_{e}^{T} \Delta_{2} \mathbf{z}_{2} \\
& -\mathbf{z}_{2}^{T} \Delta_{2} \mathbf{J}_{e} \mathbf{M}^{-}{ }^{\prime} \overline{\mathbf{R}}_{*}{ }^{1} \mathbf{M}{ }^{1} \mathbf{J}_{\varepsilon}^{T} \Delta_{2} \mathbf{z}_{2}+2 \mathbf{z}_{2}^{T} \Delta_{2} \overline{\mathbf{N}}_{1} \mathbf{z}_{1}+2 \mathbf{z}_{2}^{T} \Delta_{2} \overline{\mathbf{N}}_{2} \mathbf{z}_{2} .
\end{aligned}
$$


By rewriting $\mathbf{z}^{T} \overline{\mathbf{Q}} \mathbf{z}$ as:

$$
\mathbf{z}^{T} \overline{\mathbf{Q}}_{\mathbf{z}}=\mathbf{z}_{1}^{T} \overline{\mathbf{Q}}_{11} \mathbf{z}_{1}+2 \mathbf{z}_{2}^{T} \overline{\mathbf{Q}}_{12} \mathbf{z}_{1}+\mathbf{z}_{1}^{T} \overline{\mathbf{Q}}_{22} \mathbf{z}_{2},
$$

completing the squares for:

$$
\begin{gathered}
-\mathbf{z}_{1}^{T} \overline{\mathbf{Q}}_{11} \mathbf{z}_{1}+2 \mathbf{z}_{2}^{T}\left(\Delta_{2} \overline{\mathbf{N}}_{1}-\overline{\mathbf{Q}}_{12}\right) \mathbf{z}_{1}=-\left\|\mathbf{z}_{1}-\overline{\mathbf{Q}}_{11}^{-1}\left(\Delta_{2} \overline{\mathbf{N}}_{1}-\overline{\mathbf{Q}}_{12}\right) \mathbf{z}_{2}\right\|_{\overline{\mathbf{Q}}_{11}}^{2} \\
+\mathbf{z}_{2}^{T}\left(\overline{\mathbf{N}}_{1}^{T} \Delta_{2} \overline{\mathbf{Q}}_{11}^{-1} \Delta_{2} \overline{\mathbf{N}}_{1}-\overline{\mathbf{N}}_{1}^{T} \Delta_{2} \overline{\mathbf{Q}}_{11}^{-1} \overline{\mathbf{Q}}_{12}-\overline{\mathbf{Q}}_{12}^{T} \overline{\mathbf{Q}}_{11}^{-1} \Delta_{2} \overline{\mathbf{N}}_{1}\right) \mathbf{z}_{2}+\mathbf{z}_{2}^{T} \overline{\mathbf{Q}}_{12}^{T} \overline{\mathbf{Q}}_{11}^{-1} \overline{\mathbf{Q}}_{12} \mathbf{z}_{2}
\end{gathered}
$$

and introducing:

$$
\begin{aligned}
\bar{\Sigma}_{o}(\mathbf{z})= & \overline{\mathbf{N}}_{1}^{T} \Delta_{2} \overline{\mathbf{Q}}_{11}^{-1} \Delta_{2} \overline{\mathbf{N}}_{1}+\Delta_{2} \overline{\mathbf{N}}_{2}+\overline{\mathbf{N}}_{2}^{T} \Delta_{2}-\overline{\mathbf{N}}_{1}^{T} \Delta_{2} \overline{\mathbf{Q}}_{11}^{-1} \overline{\mathbf{Q}}_{12} \\
& -\overline{\mathbf{Q}}_{12}^{T} \overline{\mathbf{Q}}_{11}^{-1} \Delta_{2} \overline{\mathbf{N}}_{1}-\frac{1}{\gamma^{2}} \Delta_{2} \mathbf{M}^{-2} \Delta_{2}+\frac{1}{\gamma^{2}} \Delta_{2} \mathbf{J}_{e} \mathbf{M}^{-2} \mathbf{J}_{e}^{T} \Delta_{2},
\end{aligned}
$$

which satisfies $\bar{\Sigma}_{o}(\mathbf{0})=\mathbf{0}$, we obtain:

$$
\begin{aligned}
\dot{\bar{V}}= & -\left\|\mathbf{z}_{1}-\overline{\mathbf{Q}}_{11}^{-1}\left(\Delta_{2} \overline{\mathbf{N}}_{1}-\overline{\mathbf{Q}}_{12}\right) \mathbf{z}_{2}\right\|_{\mathbf{Q}_{11}}^{2}-\mathbf{z}_{2}^{T}\left(\overline{\mathbf{Q}}_{22}-\overline{\mathbf{Q}}_{12}^{T} \overline{\mathbf{Q}}_{11}^{-1} \overline{\mathbf{Q}}_{12}\right) \mathbf{z}_{2} \\
& -\overline{\mathbf{u}}^{T} \overline{\mathbf{R}}_{*}(\mathbf{z}) \overline{\mathbf{u}}+\gamma^{2} \mathbf{w}^{T} \mathbf{w}+\left\|\overline{\mathbf{u}}+\overline{\mathbf{R}}_{*}^{-1} \mathbf{M}^{-1} \mathbf{J}_{e}^{T} \Delta_{2} \mathbf{z}_{2}\right\|_{\overline{\mathbf{R}}_{*}(\mathbf{z})}^{2} \\
& -\gamma^{2}\left\|\mathbf{w}-\frac{1}{\gamma^{2}} \mathbf{J}_{e} \mathbf{M}^{-1} \mathbf{J}_{e}^{T} \Delta_{2} \mathbf{z}_{2}\right\|^{2}-\varphi^{T}\left(\overline{\mathbf{R}}_{*}^{-1}(\mathbf{z})-\overline{\mathbf{R}}_{o}^{-1}\left(\mathbf{z}_{1}\right)-\bar{\Sigma}(\mathbf{z})\right) \varphi
\end{aligned}
$$

where

$$
\begin{aligned}
\varphi & =\mathbf{M}^{-1} \mathbf{J}_{e}^{T} \Delta_{2} \mathbf{z}_{2}, \\
\overline{\mathbf{R}}_{o}^{-1}\left(\mathbf{z}_{1}\right) & =\mathbf{M} \mathbf{J}_{e}^{T} \mathbf{M}^{-1} \mathbf{R}^{-1} \mathbf{M}^{-1} \mathbf{J}_{e} \mathbf{M} \\
\bar{\Sigma}(\mathbf{z}) & =\mathbf{M} \mathbf{J}_{e}^{T} \Delta_{2}^{-1} \bar{\Sigma}_{o}(\mathbf{z}) \Delta_{2}^{-1} \mathbf{J}_{e} \mathbf{M} .
\end{aligned}
$$

If the control law is chosen according to:

$$
\overline{\mathbf{u}}=\overline{\mathbf{R}}_{*}^{-1}(\mathbf{z}) \mathbf{M}^{-1} \mathbf{J}_{c}^{T} \Delta_{2} \mathbf{z}_{2}
$$

and $\overline{\mathbf{R}}_{*}(\mathbf{z})$ is chosen such that:

$$
\overline{\mathbf{R}}_{*}^{-1}(\mathbf{z}) \geq \overline{\mathbf{R}}_{o}^{-1}\left(\mathbf{z}_{1}\right)+\bar{\Sigma}(\mathbf{z})
$$

then

$$
\begin{aligned}
\dot{\bar{V}} & =-\bar{q}(\mathbf{z})-\overline{\mathbf{u}}^{T} \overline{\mathbf{R}}_{*}(\mathbf{z}) \overline{\mathbf{u}}+\gamma^{2} \mathbf{w}^{T} \mathbf{w}-\gamma^{2}\left\|\mathbf{w}-\frac{1}{\gamma^{2}} \mathbf{J}_{e} \mathbf{M}^{-1} \mathbf{J}_{e}^{T} \Delta_{2} \mathbf{z}_{2}\right\|^{2} \\
& \leq-\bar{q}(\mathbf{z})-\overline{\mathbf{u}}^{T} \overline{\mathbf{R}}_{*}(\mathbf{z}) \overline{\mathbf{u}}+\gamma^{2} \mathbf{w}^{T} \mathbf{w}
\end{aligned}
$$

where

$$
\begin{aligned}
\bar{q}(\mathbf{z})= & \left\|\mathbf{z}_{1}-\overline{\mathbf{Q}}_{11}^{-1}\left(\Delta_{2} \overline{\boldsymbol{\eta}}_{1}-\overline{\mathbf{Q}}_{12}\right) \mathbf{z}_{2}\right\|_{\mathbf{Q}_{11}}^{2}+\mathbf{z}_{2}^{T}\left(\overline{\mathbf{Q}}_{22}-\overline{\mathbf{Q}}_{12}^{T} \overline{\mathbf{Q}}_{11}^{-1} \overline{\mathbf{Q}}_{12}\right) \mathbf{z}_{2} \\
& +\varphi^{T}\left(\overline{\mathbf{R}}_{*}^{-1}(\mathbf{z})-\overline{\mathbf{R}}_{o}^{-1}(\mathbf{z})-\overline{\mathbf{\Sigma}}(\mathbf{z})\right) \varphi .
\end{aligned}
$$

From (325) it is clear that the system is input-to-state stable. In the absence of disturbances, $\mathbf{w} \equiv \mathbf{0}$, the equilibrium $\mathbf{z}=0$ is rendered GAS and LES. Moreover, the dual objective of local optimality and global inverset optimality is achieved with respect to the cost functional:

$$
J=\int_{0}^{\infty}\left(\bar{q}(\mathbf{z})+\overline{\mathbf{u}}^{T} \overline{\mathbf{R}}_{*}(\mathbf{z}) \overline{\mathbf{u}}-\gamma^{2} \mathbf{w}^{T} \mathbf{w}\right) d t
$$

where $\bar{q}(\mathbf{z})$ and $\overline{\mathbf{R}}_{*}(\mathbf{z})$ are selected to satisfy the locally optimal requirements. 
One possible choice for $\overline{\mathbf{R}}_{*}(\mathbf{z})$ that makes sure that the matrix is invertible and continuous for all $\mathbf{z}$ is:

$$
\overrightarrow{\mathbf{R}}_{*}^{-1}(\mathbf{z})=\mu_{1}(\mathbf{z}) \mathbf{I}+\mu_{2}(\mathbf{z}) \overline{\mathbf{R}}_{o}^{-1}(\mathbf{z})
$$

where

$$
\begin{aligned}
& \mu_{1}(\mathbf{z})=\max \left\{0, \lambda_{1}(\mathbf{z})\right\}, \\
& \mu_{2}(\mathbf{z})=\left\{\begin{array}{cc}
1 \quad: & \lambda_{1}(\mathbf{z}) \geq-\lambda_{2}(\mathbf{z}) \\
\left(1+\left|\lambda_{1}(\mathbf{z})+\lambda_{2}(\mathbf{z})\right|^{k}\right)^{-1}: & \text { otherwise }
\end{array}\right. \\
& \lambda_{1}(\mathbf{z})=\lambda_{\max }(\bar{\Sigma}(\mathbf{z})), \quad \lambda_{2}(\mathbf{z})=\lambda_{\max }\left(\overline{\mathbf{R}}_{o}^{-1}(\mathbf{z})\right)
\end{aligned}
$$

for any $k \geq 0$. Here, $\lambda_{\max }(\cdot)$ denotes the largest eigenvalue of $\overline{\mathbf{R}}_{o}^{-1}(\mathbf{z})$ and $\bar{\Sigma}(\mathbf{z})$ for the current value of $\mathbf{z}$. By this choice of $\varepsilon_{2}(\mathbf{z})$ the control effect will be reduced in cases where the nonlinearities are helpful for stability.

Comment: This example is equivalent to the MIMO mass-damper-spring example in Chapter 2 if:

$$
\mathbf{J}_{e}=\mathbf{I}, \quad \overline{\mathbf{u}}=\mathbf{B u}, \quad \mathbf{H}(\mathbf{x})=\mathbf{0}
$$

\section{Appendix A. Stability Theory}

In this appendix we will briefly review some useful results for stability and convergence:

- Appendix A.1: Lyapunov stability of nonlinear autonomous systems $\dot{\mathbf{x}}=\mathbf{f}(\mathbf{x})$, that is systems where $\mathbf{f}(\mathbf{x})$ does not explicitly depend on the time $t$.

- Appendix A.2: Lyapunov stability of nonlinear non-autonomous systems $\dot{\mathbf{x}}=\mathbf{f}(\mathbf{x}, t)$, that is systems where $\mathbf{f}(\mathbf{x}, t)$ does depend on $t$ explicitly.

\section{A.1. Lyapunov Stability for Autonomous Systems}

Before stating the main Lyapunov theorems for autonomous systems we will briefly review the concepts of stability and convergence (Khalil, 1996).

\section{A.1.1. Stability and Convergence}

Consider the nonlinear time-invariant system:

$$
\dot{\mathbf{x}}=\mathbf{f}(\mathbf{x}), \mathbf{x}(0)=\mathbf{x}_{0}
$$

where $\mathbf{x} \in \mathfrak{R}^{n}$ and $\mathbf{f}: \mathfrak{R}^{n} \rightarrow \mathfrak{R}^{n}$ is assumed to be locally Lipschitz in $\mathbf{x}$, that is:

$$
\|\mathbf{f}(\mathbf{x})-\mathbf{f}(\mathbf{y})\| \leq L\|\mathbf{x}-\mathbf{y}\|, \forall \mathbf{x}, \mathbf{y}
$$

where $L$ can be interpreted as the Lipschitz constant. Let $\mathbf{x}_{e}$ denote the equilibrium point of (A.1) given by:

$$
\mathbf{f}\left(\mathbf{x}_{e}\right)=\mathbf{0}
$$

The solutions of $\mathbf{x}(t)$ of (A.1) are:

- bounded, if there exist a non-negative function $\gamma(\mathbf{x}(t))$ such that:

$$
\|\mathbf{x}(t)\| \leq \gamma(\mathbf{x}(t)), \quad \forall t \geq 0
$$


In addition, the equilibrium point $\mathbf{x}_{e}$ of (A.1) is:

- stable, if, for each $\epsilon>0$, there exists a $\delta(\epsilon)$ such that:

$$
\|\mathbf{x}(0)\|<\delta(\epsilon) \Rightarrow\|\mathbf{x}(t)\|<\epsilon, \quad \forall t \geq 0
$$

- unstable, if it is not stable.

- attractive, if there exists an $r(\mathbf{x}(t))$ such that:

$$
\|\mathbf{x}(0)\|<r(\mathbf{x}(t)) \Rightarrow \lim _{t \rightarrow \infty}\|\mathbf{x}(t)\|=0
$$

Moreover, attractivity implies convergence of $\mathbf{x}(t) \rightarrow 0$.

- (locally) asymptotically stable (AS), if the equilibrium point $\mathbf{x}_{e}$ is stable and attractive.

- globally stable (GS), if the equilibrium point $\mathbf{x}_{e}$ is stable and if all the solutions $\mathbf{x}(t)$ of (A.1) are bounded.

- global asymptotically stable (GAS), if the equilibrium point $\mathbf{x}_{e}$ is stable for all $\mathbf{x}(0)$ (region of attraction $\mathfrak{R}^{n}$ ).

- (locally) exponentially stable (ES), if there exist positive constants $\alpha, \lambda$ and $r$ such that:

$$
\|\mathbf{x}(0)\|<r \Rightarrow\|\mathbf{x}(t)\|<\alpha \exp (-\lambda t)\|\mathbf{x}(0)\|, \quad \forall t \geq 0
$$

- globally exponential stable (GES), if there exist positive constants $\alpha, \lambda$ and $r$ such that for all $\mathbf{x}(0)$ (region of attraction $\mathfrak{R}^{n}$ ).

$$
\|\mathbf{x}(t)\|<\alpha \exp (-\lambda t)\|\mathbf{x}(0)\|, \quad \forall t \geq 0
$$

Different theorems for investigation of stability and convergence will now be presented. A guideline for which theorem should be chosen is given in Table A.1 whereas the different theorems are listed in the forthcoming sections.

Notice that for non-autonomous systems GAS is replaced by global uniform asymptotic stability (GUAS) since uniformity is a necessary requirement in the case of time-varying nonlinear systems (Khalil, 1996).

\section{A.1.2. Lyapunov's Direct Method}

Theorem 3 (Lyapunov's Direct Method) Let $\mathbf{x}_{e}$ be the equilibrium point of (A.1) and assume that $\mathbf{f}(\mathbf{x})$ is locally Lipschitz in $\mathbf{x}$. Let $V: \Re^{n} \rightarrow \mathfrak{R}_{+}$be a continuously differentiable function $\mathbf{V}(\mathbf{x})$ satisfying:

- $V(\mathbf{x})>0$ (positive definite) and $V(0)=0$

- $\dot{V}(\mathbf{x})=\frac{\partial V(\mathbf{x})}{\partial \mathbf{x}} \mathbf{f}(\mathbf{x}) \leq-W(\mathbf{x}) \leq 0$

\begin{tabular}{|c|c|c|c|}
\hline $\begin{array}{l}\text { Autonomous } \\
\text { systems }\end{array}$ & $\begin{array}{l}V>0, \dot{V}<0 \\
V>0, \dot{V} \leq 0\end{array}$ & $\begin{array}{l}\text { Lyapunov's direct method } \\
\text { LaSalle's theorem }\end{array}$ & $\begin{array}{l}\text { GAS } \\
\text { GAS }\end{array}$ \\
\hline $\begin{array}{l}\text { Non-autonomous } \\
\text { systems }\end{array}$ & $\begin{array}{l}V>0, \dot{V}<0 \\
V \geq 0, \dot{V} \leq 0\end{array}$ & $\begin{array}{l}\text { LaSalle-Yoshizawa's theorem } \\
\text { Barbalat's lemma }\end{array}$ & $\begin{array}{l}\text { GUAS } \\
\text { convergence }\end{array}$ \\
\hline
\end{tabular}

- $V(\mathbf{x}) \rightarrow \infty$ as $\|\mathbf{x}\| \rightarrow \infty$ (radially unbounded)

Table A1. Classification of theorems for stability and convergence 
then the equilibrium point $\mathbf{x}_{e}$ is $G S$ if $W(\mathbf{x}) \geq 0$ (positive semi-definite) and GAS if $W(\mathbf{x})>0$ (positive definite).

The requirement that $W(\mathbf{x})>0$ (or $\dot{V}(\mathbf{x})<0)$ is in many cases difficult to satisfy. This is illustrated in the following example.

Example 4 (Stability of a Mass-Damper-Spring System) Consider the massdamper-spring system:

$$
\begin{aligned}
\dot{x} & =v \\
m \dot{v}+d(v) v+k x^{2} & =0
\end{aligned}
$$

where $m>0, d(v)>0, \forall v$ and $k>0$, see Figure A.1.

Choosing $V(\mathbf{x})$ as the sum of kinetic energy $\frac{1}{2} m v^{2}$ and potential energy $\frac{1}{2} k x^{2}$ :

$$
V(\mathbf{x})=\frac{1}{2}\left(m v^{2}+k x^{2}\right)=\frac{1}{2} \mathbf{x}^{T}\left[\begin{array}{cc}
m & 0 \\
0 & k
\end{array}\right] \mathbf{x}
$$

where $\mathbf{x}=[v, x]^{T}$, results in

$$
\begin{aligned}
\dot{V}(\mathbf{x}) & =m v \dot{v}+k x \dot{x} \\
& =v(m \dot{v}+k x) \\
& =-d(v) v^{2} \\
& =-\mathbf{x}^{T}\left[\begin{array}{cc}
d(v) & 0 \\
0 & 0
\end{array}\right] \mathbf{x}
\end{aligned}
$$

Hence, only stability can be concluded from Theorem 3 since $\dot{V}(\mathbf{x})$ is negative semi-definite. However, GAS can in many cases also be proven for systems with a negative semi-definite $\dot{V}(\mathbf{x})$ thanks to the invariant set theorem of LaSalle.

\section{A.1.3. La Salle's Invariant Set Theorem}

The theorem of LaSalle can be used to check if a nonlinear autonomous system is GAS even through $\dot{V}(\mathbf{x})$ is only negative semi-definite.

Theorem 4 (LaSalle's Invariant Set Theorem) Let $V: \mathfrak{R}^{n} \rightarrow \mathfrak{R}_{+}$be a continuously differentiable function $V(\mathbf{x})$ such that:

$$
V(\mathbf{x}) \rightarrow \infty \text { as }\|\mathbf{x}\| \rightarrow \infty
$$

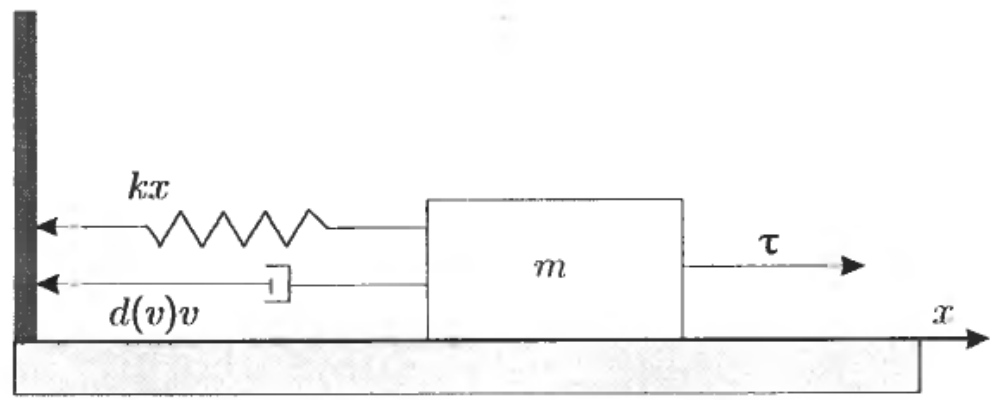

Figure 13. Mass-damper-spring system. 


$$
\dot{V}(\mathbf{x}) \leq 0, \quad \forall \mathbf{x}
$$

Let $\Omega$ be the set of all points where $\dot{V}(\mathbf{x})=0$, that is:

$$
\Omega=\left\{\mathrm{x} \in \mathfrak{R}^{n} \mid \dot{V}(\mathbf{x})=0\right\}
$$

and $M$ be the largest invariant set in $\Omega$, then all solutions $\mathbf{x}(t)$ converge to $M$ and the equilibrium point $\mathbf{x}_{e}$ of (A.1) is GAS.

Example 5 (Cont. Example 4: Stability of a Mass-Damper-Spring System) Again consider the mass-damper-spring system of Example 4 . The set $\Omega$ is found by requiring that

$$
\dot{V}(\mathbf{x})=-d(v) v^{2} \equiv 0
$$

which is true for $v=0$. Therefore:

$$
\Omega=\{(x \in \Re, v=0)\}
$$

Now $v=0$, implies that mv $=-k x$ which is non-zeroas long as $x \neq 0$. Hence, the system cannot get "stuck" at an equilibrium point value other than $x=0$. Since the equilibrium point of the mass-damper-spring system is $(x, v)=(0,0)$, the largest invariant set $M$ in $\Omega$ contains only one point, namely $(x, v)=(0,0)$. Hence, the equilibrium point of (A.I) is GAS according to Theorem 4.

\section{A.1.4. Global Exponential Stability}

When performing backstepping Lyapunov stability analysis often results in a system which not only is GAS but also globally exponentially stable (GES). The following theorem is useful to check exponential convergence.

Theorem 5 (Global Exponential Stability) Let $\mathbf{x}_{e}$ be the equilibrium point of (A.1) and assume that $\mathbf{f}(\mathbf{x})$ is locally Lipschitz in $\mathbf{x}$. Let $V: \mathfrak{R}^{n} \rightarrow \Re_{+}$be a continuously differentiable and radially unbounded function $V(\mathbf{x})$ satisfying:

$$
\begin{aligned}
& V(\mathbf{x})=\frac{1}{2} \mathbf{x}^{T} \mathbf{P} \mathbf{x} \\
& \dot{V}(\mathbf{x}) \leq-\mathbf{x}^{T} \mathbf{Q} \mathbf{x}
\end{aligned}
$$

with constant matrices $\mathbf{P}=\mathbf{P}^{T}>0$ and $\mathbf{Q}=\mathbf{Q}^{T}>0$, then the equilibrium point $\mathbf{x}_{e}$ is GES and the state vector satisfies:

$$
\|\mathbf{x}(t)\|_{2} \leq \sqrt{\frac{\lambda_{\max }(\mathbf{P})}{\lambda_{\min }(\mathbf{P})}} e^{-\frac{1}{2} \alpha\left(t-t_{0}\right)}\left\|\mathbf{x}\left(t_{0}\right)\right\|_{2}
$$

where

$$
\alpha=\frac{\lambda_{\min }(\mathbf{Q})}{\lambda_{\max }(\mathbf{P})}>\mathbf{0}
$$

is the convergence rate.

Proof. Since $V(x)$ is bounded by:

$$
0<\lambda_{\min }(\mathbf{P}) \mathbf{x}^{T} \mathbf{x} \leq V(\mathbf{x}) \leq \lambda_{\max }(\mathbf{P}) \mathbf{x}^{T} \mathbf{x}, \quad \forall \mathbf{x} \neq \mathbf{0}
$$


we have that:

$$
-\mathbf{x}^{T} \mathbf{x} \leq-\frac{1}{\lambda_{\max }(\mathbf{P})} V(\mathbf{x})
$$

Hence, it follows from (A.20) that:

$$
\begin{aligned}
\dot{V}(\mathbf{x}) & \leq-\mathbf{x}^{T} \mathbf{Q} \mathbf{x} \\
& \leq-\lambda_{\min }(\mathbf{Q}) \mathbf{x}^{T} \mathbf{x} \\
& \leq \frac{\lambda_{\min }(\mathbf{Q})}{\lambda_{\max }(\mathbf{P})} V(\mathbf{x})
\end{aligned}
$$

Integration of $\dot{V}(\mathbf{x})$ yields:

$$
V(\mathbf{x}(t)) \leq e^{-\alpha\left(t-t_{0}\right)} V(\mathbf{x}(0))
$$

Finally $\|\mathbf{x}\|_{2}=\sqrt{\mathbf{x}^{T} \mathbf{x}}$ and (A.24) implies:

$$
\|\mathbf{x}(t)\|_{2} \leq \sqrt{\frac{\lambda_{\max }(\mathbf{P})}{\lambda_{\min }(\mathbf{P})}} e^{-\frac{1}{2} \alpha\left(t-t_{0}\right)}\left\|\mathbf{x}\left(t_{0}\right)\right\|_{2}
$$

q.e.d. This shows that $\|\mathbf{x}\|_{2}$ will converge exponentially to zero with convergence rate $\alpha$

\section{A.2. Lyapunov Stability of Non-Autonomous System}

In this section we will briefly review some useful theorems for convergence and stability for time-varying nonlinear systems:

$$
\dot{\mathbf{x}}=\mathbf{f}(\mathbf{x}, t), \mathbf{x}(0)=\mathbf{x}_{0}
$$

where $\mathbf{x} \in \mathfrak{R}^{n}, t \in \mathfrak{R}_{+}$and $\mathbf{f}: \mathfrak{R}^{n} \times \mathfrak{R}_{+} \rightarrow \mathfrak{R}^{n}$ is assumed to be locally Lipschitz in $\mathbf{x}$.

\section{A.2.1. Barbalăt's Lemma}

Lemma 1 (Barbălat's Lemma) Let $\phi: \mathfrak{R}_{+} \rightarrow \mathfrak{R}$ be a uniformly continuous function and suppose that $\lim _{t \rightarrow \infty} \int_{0}^{\infty} f(\tau) d r$ exists and is finite, then:

$$
\lim _{t \rightarrow \infty} \phi(t)=0
$$

Proof. See Barbălat (1959).

Notice that Barbălat's lemma only guarantees global convergence. This result is particularly useful if there exists a uniformly continuous function $V: \mathfrak{R}^{n} \rightarrow \mathfrak{R}_{+} \rightarrow \mathfrak{R}_{+}$ satisfying:

i) $V(x, t) \geq 0$

ii) $\dot{V}(\mathbf{x}, t) \leq 0$

iii) $\dot{V}(\mathbf{x}, t)$ is uniformly continuous

Hence, according to Barbălat's lemma $\lim _{t \rightarrow \infty} \dot{V}(\mathbf{x}, t)=0$. The requirement that $\dot{V}$ should be uniformly continuous can easily be checked by using:

$$
\ddot{V}(\mathbf{x}, t) \text { is bounded } \Rightarrow \dot{V}(\mathbf{x}, t) \text { is uniformly continuous }
$$

\section{A.2.2. LaSalle-Yoshizawa's Theorem}

For non-autonomous systems the following theorem of LaSalle (1996) and Yoshizawa (1968) is quite useful: 
Theorem 6 (LaSalle-Yoshizawa's Theorem) Let $\mathbf{x}_{e}=0$ be the equilibrium point of (A.29) and assume that $\mathbf{f}(\mathbf{x}, t)$ is locally Lipschitz in $\mathbf{x}$. Let $V: \Re^{n} \times \Re_{+} \rightarrow \Re_{+}$be a continuously differentiable function $V(\mathbf{x}, t)$ satisfying:

- $V(\mathbf{x}, t)>0$ (positive definite) and $V(0)=0$

$\cdot \dot{V}(\mathbf{x}, t)=\frac{\partial V(\mathbf{x}, t)}{\partial \mathbf{t}}+\frac{\partial V(\mathbf{x}, t)}{\partial \mathbf{x}} \mathbf{f}(\mathbf{x}, t) \leq-W(\mathbf{x}) \leq 0$

- $V(\mathbf{x}, t) \rightarrow \infty$ as $\|\mathbf{x}\| \rightarrow \infty$ (radially unbounded)

where $W(\mathbf{x})$ is a continuous function, then all solutions $\mathbf{x}(t)$ of (A.29) are globally uniformly bounded and:

$$
\lim _{t \rightarrow \infty} W(\mathbf{x})=0
$$

In addition, if $W(\mathbf{x})>0$ (positive definite), then the equilibrium point $\mathbf{x}_{e}=\mathbf{0}$ of (A.29) is GUAS.

Proof. See LaSalle (1996) and Yoshizawa (1968).

REFERENCES

BARBÅlat (1959). Systèmes d'Équations Différentielles d'Oscillations Non Linéaires. Revue de Mathématiques Pures et Appliquées, Vol. 4(2), 267-270. Académie de la République Populaire Roumaine (in French).

BECH, M. I. and WAGNER SMITH, L. (1969). Analogue Simulation of Ship Maneuvers. Technical Report Hy-14, Hydro- and Aerodynamics Laboratory, Lyngby, Denmark.

ByrneS, C. I. and IsIDORI, A. (1989). New Results and Examples in Nonlinear Feedback Stabilization. Systems \& Control Letters, Vol. 12, 437-442.

Byrnes, C. I., IsIDORI, A. and Willems, J. C. (1991). Passivity, Feedback Equivalence, and the Global Stabilization of Minimum Phases Nonlinear Systems. IEEE Transactions on Automatic Control, TAC-36, 1228-1240.

EZAL, K., PAN, Z. and KOKOTOVIC, P. V. (1998). Locally Optimal Backstepping Design. Proceedings of 36th IEEE Conference on Decision and Control (CDC'97), San Diego, December 1997, pp. 1767-1773.

EZAL, K. (1998). Disturbance Attenuating Control of Nonlinear Systems with Local Optimality. $\mathrm{PhD}$ thesis, University of California, Santa Barbara.

Fossen, T. I. (1994). Guidance and Control of Ocean Vehicles, John Wiley \& Sons, Ltd., Chichester, England.

FossEn, T. I. (1999). Nonlinear Backstepping Designs: Applications to Mechanical Systems and Ship Control (in progress).

FosSEN, T. I. and BERGE, S. (1997). Nonlinear Vectorial Backstepping Design for Global Exponential Tracking of Marine Vessels in the Presence of Actuator Dynamics. Proceedings of IEEE Conference on Decision and Control (CDC'97), San Diego, December 1997, pp. 4237-4242.

FoSSEN, T.I. and FJELLSTAD, O.-E. (1995). Nonlinear Modelling of Marine Vehicles in 6 Degrees of Freedom. International Journal of Mathematical Modelling of Systems, JMMS-1(1), 17-28.

FOSSEN, T. I. and GRøVLEN, Å. (1998). Nonlinear Output Feedback Control of Dynamically Positioned Ships Using Vectorial Observer Backstepping. IEEE Transactions on Control Systems Technology, TCST-6(1), January, 121-128.

FOSSEN, T. I., LORIA, A. and TEEL, A. (1998). UGAS/ULES when Backstepping with Integral Action: Applications to Mechanical Systems and Ships. Submitted to the Journal of Nonlinear and Robust Control. 
Fossen, T. I. and StRand, J. P. (1999). Passive Nonlinear Observer Design for Ships Using Lyapunov Methods: Experimental Results with a Supply Vessel. Automatica, AUT-35(1), January.

Godhavn, J.-M., Fossen, T. I. and Berge, S. P. (1998). Nonlinear and Adaptive Backstepping Designs for Tracking Control of Ships. International Journal of Adaptive Control and Signal Processing, Special Issue on Marine Systems Control (to appear).

Gravdahl, J. T. and Egeland, O. (1998). Two Results on Compressor Surge Control with Disturbance Rejection. Proceedings of the 37th IEEE Conference on Decision and Control (CDC'98), Tampa, Florida.

Gravdahl, J. T. (1998). Surge and Rotating Stall in Compressors: Modeling and Control, Advances in Industrial Control, Springer-Verlag, London.

GreiTZER, E. M. (1976). Surge and Rotating Stall in Axial Flow Compressors, Part I: Theoretical Compression System Model. Journal of Engineering for Power, JEP-98, 190-198.

Kanellakopoulos, I., KoKotovic, P. V. and Morse, A. S. (1992). A Toolkit for Nonlinear Feedback Design. Systems \& Control Letters, Vol. 18, 83-92.

KhaLI, H. K. (1996). Nonlinear Systems, Macmillan, New York.

KoDITSCHEK, D. E. (1987). Adaptive Techniques for Mechanical Systems. Proceedings of the 5th Yale Workshop on Adaptive Systems, New Haven, CT, 259-265.

Kокотоvic, P. V. and SuSSMANN, H.J. (1989). A Positive Real Condition for Global Stabilization of Nonlinear Systems. Systems \& Control Letters, Vol. 13, 125-133.

Kокотоvic, P. V. (1991). The Joy of Feedback: Nonlinear and Adaptive, 1991 Bode Price Lecture. IEEE Control Systems Magazine, Vol. 12, 7-17.

KrStIC, M. and DENG, H. (1998). Stabilization of Nonlinear Uncertain Systems, Springer Verlag, Berlin.

Krstic, M., Kanellakopoulos, I. and Kokotovic, P. V. (1995). Nonlinear and Adaptive Control Design, John Wilcy \& Sons Ltd., New York.

LANDAU, Y. D. (1977). Adaptive Control, Marcel Dekker, New York.

LASALLE, J. P. (1966). Stability Theory for Ordinary Differential Equations. Journal of Differential Equations, Vol. 4, 57-65.

Lozano, R., Brogliato, B. and Landau, I. D. (1992). Passivity and Global Stabilization of Cascaded Nonlinear Systems. IEEE Transactions on Automatic Control, TAC-37, 1386-1388.

Narendra, K. S. and Annaswamy, A. M. (1989). Stable Adaptive Systems, Prentice Hall, Englewood Cliffs, NJ.

NorRBin, N. H. (1963). On the Design and Analyses of the Zig-Zag test on Base of Quasi Linear Frequency Response. Technical Report B 104-3, The Swedish State Shipbuilding Experimental Tank (SSPA), Gothenburg, Sweden.

ORTEGA, R. (1991). Passivity Properties for Stabilization of Cascaded Nonlinear Systems. Automatica, AUT-27, 423-424.

PARKS, P. C. (1966). Lyapunov Redesign of Model Reference Adaptive Control Systems. IEEE Transactions on Automatic Control, TAC-11, 362-367.

SABERI, A., KOKOTOVIC, P. V. and SuSSMANN, H.J. (1990). Global Stabilization of Partially Linear Composite Systems. SIAM J. Control Opt., Vol. 28, 1491-1503.

Sciavicco, L. and Siciliano, B. (1996). Modelling and Control of Robot Manipulators, McGraw-Hill Companies.

SePulchre, R., Jankovic, M. and Koкo'tovic, P. (1997). Constructive Nonlinear Control, Springer Verlag, Berlin.

Slotine, J. J. E. and LI, W. (1987). Adaptive Manipulator Control: A Case Study. Proceedings of the 1987 IEEE Robotics and Automation, Raleigh, North Carolina, pp. 1392-1400.

Sonntag, E. D. and Sussmann, H. J. (1988). Further Comments on the Stabilizability of the Angular Velocity of a Rigid Body. Systems \& Control Letters, Vol. 12, 437-442.

Strand, J. P. Nonlinear Position Control Systems Design for Marine Vessels, Doctoral Dissertation, Dept. of Eng. Cybernetics, Norwegian Univ. of Sci. and Techn., June 1999.

Strand, J. P., Ezal, K., Fossen, T. I. and Kokotovic, P. V. (1998). Nonlinear Control of Ships: A Locally Optimal Design. Proceedings of the IFAC NOLCOS"98, Enschede, The Netherlands, 1-3 July 1998.

Strand, J. P., Ezal, K., Fossen, T. I. and KukutuviC, P. V. (1999). Nonlinear Output Feedback 
Tutorial on Nonlinear Backstopping: Applications to Ship Control

135

and Locally Optimal Control of Free-Floating and Moored Ships: Experimental Results with a Model Ship (submitted to Automatica).

TSINIAS, J. (1989). Sufficient Lyapunov-Like Conditions for Stabilization. Mathematics of Control, Signals and Systems, Vol. 2, 343-357.

Yoshizawa, T. (1968). Stability Theory by Lyapunov's Second Method. The Mathematical Society of Japan.

Chang, Y., IOANNOU, P. A. and Chin, C.-C. (1996). Parameter Convergence of a new Class of Adaptive Controllers. IEEE Transactions on Automatic Control, TAC-41(10), 14891493. 